This is the accepted version of the following article: Rodríguez-Concepción, M. et al. A global perspective on carotenoids: metabolism, biotechnology, and benefits for nutrition and health in Progress in lipid research (Ed. Elsevier), vol. 70 (April 2018), p. 62-93

Which has been published in final form at DOI 10.1016/j-plipres.2018.04.004

(C) 2018. This manuscript version is made available under the CC-BY-NC-ND 4.0 license http://creativecommons.org/licenses/by-nc-nd/4.0/ 


\section{A global perspective on carotenoids: metabolism, biotechnology, and benefits for nutrition and health.}

Manuel RODRIGUEZ CONCEPCION ${ }^{\mathrm{a}, *}$, Javier AVALOS $^{\mathrm{b}}$, M. Luisa BONET ${ }^{\mathrm{c}}$, Albert BORONAT ${ }^{\mathrm{a}, \mathrm{d}}$, Lourdes GOMEZ-GOMEZ ${ }^{\mathrm{e}}$, Damaso HORNERO-MENDEZ ${ }^{f}$, M. Carmen LIMON ${ }^{\mathrm{b}}$, Antonio J. MELÉNDEZ-MARTíNEZ ${ }^{g}$, Begoña OLMEDILLA-ALONSO ${ }^{h}$, Andreu PALOU ${ }^{c}$, Joan RIBOT ${ }^{c}$, Maria J. RODRIGO', Lorenzo ZACARIAS', Changfu ZHU

a, Centre for Research in Agricultural Genomics (CRAG) CSIC-IRTA-UAB-UB, 08193 Barcelona, Spain.

b, Department of Genetics, Universidad de Sevilla, 41012 Seville, Spain.

c, Laboratory of Molecular Biology, Nutrition and Biotechnology, Universitat de les Illes Balears; CIBER Fisiopatología de la Obesidad y Nutrición (CIBERobn); and Institut d'Investigació Sanitària Illes Balears (IdISBa), 07120 Palma de Mallorca, Spain.

d, Department of Biochemistry and Molecular Biomedicine, Universitat de Barcelona, 08028 Barcelona, Spain

e, Instituto Botánico, Universidad de Castilla-La Mancha, 02071 Albacete, Spain.

f, Department of Food Phytochemistry, Instituto de la Grasa (IG-CSIC), 41013 Seville, Spain.

g, Food Color \& Quality Laboratory, Area of Nutrition \& Food Science, Universidad de Sevilla, 41012 Seville, Spain.

h, Institute of Food Science, Technology and Nutrition (ICTAN-CSIC), 28040 Madrid, Spain.

i, Institute of Agrochemistry and Food Technology (IATA-CSIC), 46980 Valencia, Spain.

j, Department of Plant Production and Forestry Science, Universitat de Lleida-Agrotecnio, 25198 Lleida, Spain

* Corresponding author: manuel.rodriguez@cragenomica.es 


\begin{abstract}
Carotenoids are lipophilic isoprenoid compounds synthesized by all photosynthetic organisms and some non-photosynthetic bacteria and fungi. With some notable exceptions, animals (including humans) do not produce carotenoids de novo but take them in their diets. In photosynthetic systems carotenoids are essential for photoprotection against excess light and contribute to light harvesting, but perhaps they are best known for their properties as natural pigments in the yellow to red range. Carotenoids can be associated to fatty acids, sugars, proteins, or other compounds that can change their physical and chemical properties and influence their biological roles. Furthermore, oxidative cleavage of carotenoids produces smaller molecules such as apocarotenoids, some of which are important pigments and volatile (aroma) compounds. Enzymatic breakage of carotenoids can also produce biologically active molecules in both plants (hormones, retrograde signals) and animals (retinoids). Both carotenoids and their enzymatic cleavage products are associated with other processes positively impacting human health. Carotenoids are widely used in the industry as food ingredients, feed additives, and supplements. This review, contributed by scientists of complementary disciplines related to carotenoid research, covers recent advances and provides a perspective on future directions on the subjects of carotenoid metabolism, biotechnology, and nutritional and health benefits.
\end{abstract}

\title{
Keywords
}

Biotechnology, carotenoid, health, metabolism, nutrition, pigment 


\section{Introduction}

Carotenoids are isoprenoid metabolites synthesized by all photosynthetic organisms (including plants, algae and cyanobacteria) and some non-photosynthetic archaea, bacteria, fungi and animals. In photosynthetic systems, carotenoids participate in light harvesting and they are essential for photoprotection. By contrast, carotenoids in non-photosynthetic tissues and organisms play a role as pigments in the yellow to red range. Carotenoids provide the autumn colors of many leaves (unmasked when chlorophylls are degraded) and they are responsible for the yellow color of corn, the orange color of carrots, pumpkin, and oranges, and the red color of tomato and watermelon, among others. In addition, carotenoids can be cleaved to produce compounds with roles as growth regulators, such as abscisic acid (ABA) and strigolactones, as well as bioactive molecules. Most animals (including humans) do not synthesize carotenoids de novo but take them in the diet and use them as essential precursors for the production of retinoids such as vitamin A. Additionally; carotenoids have been proposed to confer other health benefits whose discovery is spurring their use in functional food products. It is expected that the growing demand for natural carotenoids will boost carotenoid biotechnology as a fundamental player to meet the requirements of consumers and industry for this family of healthy pigments in the next few years. In this review, we summarize the current knowledge on carotenoids in three major areas: (1) metabolism and function, (2) crop biotechnology, and (3) nutrition and health.

\section{Chemical features}

\subsection{Structure}

\subsubsection{Classes and subclasses}

Carotenoids are isoprenoid compounds with a polyene backbone that contains a variable number of conjugated double bounds (c.d.b.), a feature that imparts carotenoids the property to absorb visible light resulting in their characteristic coloration in the yellow to red range. The Carotenoid DataBase (http://carotenoiddb.jp) provides at present information on chemical structures of 1,158 carotenoids found in 691 organisms from all domains of life [1]. Depending on the presence or absence of end rings in their structure, they are classified as cyclic or acyclic carotenoids. The numbering scheme in a cyclic and an acyclic carotenoid and the different basic end groups described in carotenoids are depicted in Figure 1 [2]. Another classification of carotenoids is based on their chemical composition. Those formed exclusively by carbon and hydrogen atoms are called carotenes, whereas carotenoids containing oxygen are known as xanthophylls. Oxygenated radicals common in dietary xanthophylls are hydroxyl (lutein, zeaxanthin), epoxide (violaxanthin, neoxanthin) or carbonyl (canthaxanthin, capsanthin) groups (Figure 2). However, other oxygenated groups can be found in natural carotenoids, including carboxylic, acetate, lactone, or sulphate groups [2, 3].

Apart from these two general classifications, subclasses of carotenoids can be established considering their number of carbons and double bonds [3]. Typical carotenoids contain 40 carbon atoms (C40) and are formed by the condensation of eight C5 isoprenoid units (see Section 2 below). While $\mathrm{C} 40$ carotenoids are the most abundant in nature, some carotenoids are shorter (C30) or longer (C45 or C50). C30 carotenoids only contain six C5 isoprenoid units, whereas C45 and C50 carotenoids contain nine or ten isoprenoid units, respectively. One example is decaprenoxanthin, a C50 carotenoid (Figure 2). Furthermore, carotenoids can be cleaved to lose fragments at one or both ends of the molecule, hence generating apocarotenoids such as crocetin, a C20 compound (Figure 2). The term apocarotenoid is widely used in the literature to refer to all carotenoid cleavage products, but this is not strictly correct. Non-apocarotenoid carotenoid cleavage products include norcarotenoids, which lack one, two or three carbon atoms in the central hydrocarbons skeleton. An example is peridinin (Figure 2). Another subclass is that of secocarotenoids, in which a bond between adjacents carbons (except carbons 1 and 6 in rings) is broken. An example is semi- $\beta$-carotenone (Figure 2 ). While typical carotenoids contain a 
double bond between the carbons 15 and $15^{\prime}$, the series of conjugated double bonds is shifted in retrocarotenoids such as rhodoxanthin (Figure 2).

\subsubsection{Geometrical and optical isomers}

Given the presence of double bonds in carotenoid molecules, multiple geometrical (cis/trans or Z/E) isomers could be formed, although in most cases there are important steric hindrances [4]. Isomers cis and trans differ considerably in shape (Figure 3). Normally, natural carotenoids are mostly in their all-trans configuration, which seems to be the most stable. However, this might not be the case of acyclic carotenoids such as lycopene, phytoene or phytofluene $[5,6]$. Although the presence of cis isomers in foods and other matrices can be due to isomerization caused by heat or light $[7,8]$, it is well established that some can also occur naturally. A relevant example is (15-cis)-phytoene, which is the predominant phytoene isomer in carotenogenic organisms [9]. In addition, a small but biologically relevant proportion of some carotenoids must be in the cis configuration to be functional in the light-harvesting complex as well as in the formation of carotenoid-derived hormones. Thus, strigolactones are produced from (9-cis)- $\beta$-carotene and ABA is derived from 9-cis isomers of violaxanthin and neaxanthin (Figure 4). Other examples are the accumulation of the highly sterically hindered $\left(7,9,7^{\prime}, 9^{\prime}\right)$-tetra-cis isomer of lycopene (known as prolycopene) in the tomato tangerine mutant [10] and the natural occurrence of diverse cis isomers of phytofluene, antheraxanthin and violaxanthin in some fruits [5, 11-14]. Analyzing the presence of geometrical isomers of carotenoids in natural sources and foods is important as they exhibit different properties that may have an impact on functionality and bioavailability $[15,16]$ and can also be markers of certain technological treatments [17].

Many carotenoids contain chiral centers in their molecules, and hence optical isomers can occur. A typical example is zeaxanthin (Figure 3$)$. Two optical isomers, namely $\left(3 R-3^{\prime} R\right)$ zeaxanthin and (3R-3'S)-zeaxanthin (meso-zeaxanthin) are found in the macula lutea of the human retina [18]. Strikingly, there are not good known dietary sources of meso-zeaxanthin, although it is present in egg yolks of hens fed with this optical isomer [19]. Indeed, mesozeaxanthin is thought to be formed from lutein in the retina [20].

\subsubsection{Association with other molecules}

Carotenoids can be associated with other molecules, including fatty acids, sugars or even proteins. Xanthophylls are typically found esterified with fatty acids in many fruits [21-24] and other plant organs such as flowers [25] and tubers [26]. Similarly, there are carotenoids associated to sugar moieties, as it is the case of crocetin [27]. On the other hand, some carotenoids can form complexes with proteins (carotenoproteins) that are watersoluble and appear to stabilize carotenoids [28].

\subsection{Physicochemical properties}

With very few exceptions, carotenoids are very lipophilic, and they are usually found in hydrophobic milieus [2]. Their esterification with fatty acids further increases their lipophilicity, whereas their association with proteins or sugars decreases it. But the main feature of carotenoids is their conjugated double bond system, which is the main responsible for their color (chromophore), shape, reactivity, and photochemical properties (essential in photosynthesis, see Section 2). Most carotenoids absorb blue and violet light $(400-500 \mathrm{~nm})$ and hence exhibit colors in the yellow to red range. The relationship between structural features and color changes has been studied in common food carotenoids [13]. The color of carotenoids depends on factors such as concentration, aggregation or interaction with proteins [2,13]. Carotenoproteins can exhibit blue, purple, green, and brown colors [29]. For example, the carotenoprotein $\alpha$-crustacyanin absorbs at $632 \mathrm{~nm}$ and exhibits a blue color, while the associated carotenoid (astaxanthin) absorbs maximally at $478 \mathrm{~nm}$ and is reddish when isolated [30]. The polyene chain can also determine the shape of the carotenoid molecule. For example, it is responsible for the rigid and rod-like shape of the (all-trans)-isomers of carotenoids [2]. The unique properties of phytoene 
and phytofluene (Figure 2) mostly result from their fewer number of conjugated double bonds, which makes them colorless [31] and less rigid [32]. Both carotenes also exhibit different antioxidant capacity [33] as the conjugated double bond system is rich in electrons and it largely influences the antioxidant or pro-oxidant activities of carotenoids.

\section{Metabolism and function}

\subsection{Carotenoids in plants}

\subsubsection{Biological functions}

Carotenoids have both primary (i.e. essential) and secondary (i.e. specialized) functions in plants. Because carotenoids are indispensable for photoprotection against photooxidative damage, complete absence of carotenoids, e.g. by loss of function of PSY activity [34], results in an albino phenotype and a developmental block in the light. Carotenoids mitigate the harmful effects associated to strong light by dissipating the excess energy as heat (non-photochemical quenching, NPQ), by scavenging free radicals, and by protecting membranes from lipid peroxidation [35]. The regulation of NPQ is intrinsically linked to the xanthophyll cycle. The violaxanthin-antheraxanthin-zeaxanthin cycle (VAZ cycle) is the predominant xanthophyll cycle in most model plants, whereas many neotropical and woody plant species use the lutein epoxide (LXL) cycle [36-38] (Figure 4). Photoprotective functions are critical for the adaptation of plants to different light conditions and changing environments. Carotenoids are also part of the reaction center of photosystem II, playing a role in the assembly of pigment-protein complexes and the electron flow cycle to chlorophyll [39]. Another primary function of carotenoids is to serve as precursors of phytohormones such as ABA and strigolactones, which are key regulators of growth, development, and stress responses in plants [40]. Carotenoid-derived molecules also play roles as signals for plastid-to-nucleus and long-distance communication in plants. The identity of most of these signals remains to be identified (see Section 2.3 below).

As secondary metabolites, carotenoids play important roles in the communication of plants and animals. Their colors contribute to attract pollinators (in the case of flowers) and seed dispersing animals (in fruits) [41, 42]. This role is also fulfilled by carotenoid cleavage products which function as water-soluble pigments (e.g. bixin) and volatile aromas (e.g. $\beta$-ionone).

\subsubsection{Biosynthetic pathway}

In plants, carotenoids are $\mathrm{C} 40$ isoprenoids synthesized in plastids. Their biosynthesis and metabolism have been covered in recent reviews [43-45]. Carotenoids are formed by the condensation of the universal C5 isoprenoid precursors isopentenyl diphosphate (IPP) and dimethylallyl diphosphate (DMAPP). These precursors are produced by two independent pathways in plants: the mevalonate (MVA) pathway and the methylerythritol 4-phosphate (MEP) pathway (Figure 4). In the MVA pathway IPP is formed in the cytosol from three molecules of acetyl-COA and then isomerized to DMAPP, whereas both IPP and DMAPP are formed in the plastid from pyruvate and glyceraldehyde 3-phosphate by the MEP pathway [46]. Plant carotenoids are produced from MEP-derived precursors in plastids (Figure 4). Different lines of evidence support a limiting role of the MEP pathway for carotenoid biosynthesis [43]. Metabolic flux analyses have shown that the first enzyme of the MEP pathway, deoxyxylulose 5-phosphate synthase (DXS), is also the one with the highest flux control coefficient [47]. DXS activity is highly regulated at multiple levels [48]. Besides the primary control derived from the regulation of gene expression, a fine control is exerted within plastids through the regulation of enzyme levels. Within plastids, DXS is prone to misfolding and aggregation. Under normal growth conditions, non-functional forms of the enzyme are recognized by a specific J-protein and delivered to degradation by the stromal Clp protease complex [49-51]. Under stress conditions, however, a refolding pathway is induced to reactivate the enzyme [50,52]. DXS activity can also be repressed by a feedback mechanism involving IPP and DMAPP $[34,53]$. Other enzymes of the MEP pathway 
are regulated by the Clp protease $[49,54]$ but also by redox signals through direct interaction with thioredoxin [48].

Condensation of three IPP and one DMAPP molecules generates geranylgeranyl diphosphate (GGPP), the direct metabolic precursor not only for carotenoids but also for several other plastidial isoprenoids with important functions in photosynthesis (chlorophylls, tocopherols, plastoquinones, and phylloquinones), growth regulation (gibberellins), or environmental interactions (diterpenes), among others (Figure 4). Thus, different metabolic pathways compete for the GGPP available in the plastids $[55,56]$. Plastidial GGPP synthase (GGPPS) isoforms physically interact with enzymes that use GGPP for the production of downstream products, including carotenoids $[55,57]$. However, overexpression of GGPPproducing enzymes from different sources has shown only slight effects on plant carotenoids levels [57-60].

The first committed step of plant carotenoid biosynthesis is the condensation of two C20 GGPP molecules to form C40 15-cis-phytoene (Figure 4). This step, catalyzed by the enzyme phytoene synthase (PSY), is generally accepted to be the main rate-determining reaction of the pathway and to control the metabolic flux to carotenoids [43, 61]. PSY is typically encoded by small gene families that are differentially expressed in response to developmental and environmental signals [44]. Transcription factors involved in the regulation of PSY-encoding genes have been identified in different plant species $[41,62]$. PSY is also regulated at the enzyme level in response to a variety of factors, including plastidial sublocalization $[63,64]$ (see Section 2.1.3 below). Similar to DXS and other MEP pathway enzymes, the levels and activity of PSY and other carotenoid pathway enzymes can also be regulated by the Clp protease $[65,66]$, a mechanism that likely contributes to coordinate carotenoid biosynthesis with the supply of their metabolic precursors. Other mechanisms regulating PSY activity in different plant systems are (1) direct interaction with the ORANGE (Or) chaperone $[67,68]$, (2) direct interaction with the STAY-GREEN protein [69], and (3) negative feedback by downstream carotenoids [70, 71].

In plant cells, 15-cis-phytoene is converted to all-trans-lycopene by sequential desaturation and isomerization reactions (Figure 4). Desaturations are catalyzed by phytoene desaturase (PDS) and $\zeta$-carotene desaturase (ZDS). In concert with these steps, $\zeta$-carotene isomerase (ZISO) catalyzes the cis to trans conversion of the product of PDS, whereas carotenoid isomerase (CRTISO) transforms tetra-cis lycopene (prolycopene) into all-trans-lycopene (Figure 4). The somewhat unexpected discovery of ZISO is a good example of how incomplete is our knowledge of the carotenoid pathway even after decades of study [72]. ZISO remained unknown in part because its isomerization activity can be compensated by the photoisomerization of carotenoids in green tissues, similar to that reported for CRTISO [44]. Both PDS and ZDS, which are important to control flux though the carotenoid pathway $[73,74]$, require the operation of an electron transfer process involving the plastidial terminal oxidase (PTOX) and plastoquinone. The plastoquinone pool available for carotenoid biosynthesis has been associated to electron transport processes linked to chlororespiration and chromorespiration $[75,76]$. In turn, chromorespiration has also been related with the supply of ATP required for carotenoid biosynthesis in non-photosynthetic plastids $[75,77]$. The recent report that ZISO requires a redox-regulated heme cofactor for activity [78] highlights the relevance of the redox status of the plastids in the regulation of the carotenoid pathway.

The cyclization of lycopene is a critical step for the synthesis of carotenoids since it is the first branching step of the pathway (Figure 4). The action of lycopene $\varepsilon$-cyclase (LCYE) or lycopene $\beta$-cyclase (LCYB or CYCB, the latter term often used for chromoplast-associated isoforms such as the one expressed in tomato ripening fruit) generates carotenoids with $\varepsilon$ - or $\beta$ ionone rings, respectively. The cyclization of both ends of the linear lycopene molecule by LCYB generates $\beta$-carotene, while the coordinated action of LCYE and LCYB produces $\alpha$-carotene (Figure 4). Cyclization of both ends of lycopene with $\varepsilon$ rings is very uncommon in nature. Genes encoding LCYB and LCYE share significant sequence identity, suggesting that they may have originated by duplication of a common ancestor. Moreover, specific signatures have been 
identified in their sequences that appear to be responsible for the cyclization at one or other end of lycopene [45]. The coordinated operation of LCYE and LCYB cyclases plays a major role in the regulation of the metabolic flux of the carotenoid pathway to either the $\beta, \beta$ or the $\beta, \varepsilon$ branch $[44,79]$.

Xanthophylls are oxygenated carotenoids derived from $\alpha$ - or $\beta$-carotene (Figure 4). They are among the main carotenoids in photosynthetic tissues. Xanthophyll formation requires ringspecific hydroxylation reactions. In the case of $\beta$-carotene, two sequential hydroxylation of the $\beta$ rings produce first $\beta$-cryptoxanthin and then zeaxanthin (Figure 4). This reaction is normally catalyzed by carotene $\beta$-hydroxylase enzymes of the non-heme di-iron $(\mathrm{BCH})$ type $[43,44,70$, 80]. While $\mathrm{BCH}$ enzymes can also participate in the biosynthesis of lutein from $\alpha$-carotene, the hydroxylation the $\beta$ ring of $\alpha$-carotene to produce zeinoxanthin is usually catalyzed by CYP97A, a heme-containing cytochrome P450 enzyme $[43,44,70,80]$. A similar enzyme, CYP97C, acts as a carotene $\varepsilon$-hydroxylase to transform zeinoxanthin into lutein (Figure 4). Alternatively, CYP97C can first hydroxylate the $\varepsilon$ ring of $\alpha$-carotene to produce $\alpha$-cryptoxanthin and then CYP97A can hydroxylate the $\beta$ ring of $\alpha$-cryptoxanthin to produce lutein (Figure 4).

Zeaxanthin epoxidase (ZEP) introduces epoxy groups in the rings of zeaxanthin, resulting in the sequential formation of antheraxanthin and violaxanthin (Figure 4). These reactions can be reverted by violaxanthin de-epoxidase (VDE). Interconversion of zeaxanthin and violaxanthin is known as the VAZ cycle (Figure 4). In plants with the LxL cycle, lutein can be converted into lutein epoxide (and vice versa) by particular ZEP and VDE enzymes [38]. The introduction of an allenic double bound in the violaxanthin molecule produces neoxanthin in a step catalyzed by neoxanthin synthase (NSY). The identity of the enzyme and the mechanism of action, however, remain unclear.

In most fruits and flowers, as well as in many seeds and some tubers, xanthophylls are mono- or diesterified with different fatty acids $[24,81,82]$. This modification increases their lipophilicity and stability. The increased lipophilicity conferred by the acyl chains may favor their sequestration in the hydrophobic core of plastoglobules, thylakoid-associated lipoprotein structures enclosed by a lipid monolayer which are a major site for the massive storage of esterified carotenoids [83]. An esterase-related protein, referred to as PYP (Pale Yellow Petal), has been identified as the enzyme responsible for xanthophyll esterification in tomato flowers [84]. The co-localization of xanthophyll biosynthetic enzymes and PYP in plastoglobules may allow an efficient esterification process. At present very little is known about the regulatory role of xanthophyll acyltransferases in carotenoid accumulation. Some experiments have shown that xanthophyll acyltransferases are very selective for the acyl moiety and present a marked regioselectivity when asymmetric xanthophylls, such as lutein, are esterified [81]. The acyl donor molecules involved in the xanthophyll esterification process remain unidentified.

\subsubsection{Enzyme distribution within plastids}

The subplastidial localization and organization of carotenogenic enzymes is an essential question that remains little explored despite its relevance to fully understand carotenoid biosynthesis in plants. A previously published model [85] proposing the formation of multienzymatic complexes for carotenoid biosynthesis channeling has been recently supported by new evidence [43, 63, 78, 79, 86-88]. Carotenoid biosynthetic enzymes appear to be either associated to envelope and thylakoid membranes (PSY, PDS, ZDS, CRTISO, LCYB, LCYE, CYP97A and CYP97C) or integral membrane proteins (ZISO, BCH) [43, 63]. VDE attaches to the thylakoid membrane at acidic $\mathrm{pH}$ but behaves as a soluble lumenal protein at neutral $\mathrm{pH}$ [89]. PSY has also been found in plastoglobules or the stroma of chloroplasts from different species [64, 90], while in the plastids of dark-germinated seedlings (i.e. etioplasts) the enzyme was mainly associated to prolamellar bodies, membrane aggregations of semicrystalline structures often arranged in a geometric configuration [91]. Interestingly, PSY localization in prolamellar bodies was linked to low enzymatic activity while association to thylakoid membranes during the de-etiolation process resulted in a substantial increase in activity [91]. 
When chloroplasts differentiate into chromoplasts (plastids specialized in carotenoid biosynthesis and storage), synthesis of carotenoids is tightly associated to plastoglobules or other membranous structures derived from thylakoidal disassembly. It is common that particular isoforms of carotenoid biosynthetic enzymes are preferentially expressed in chromoplastcontaining tissues. In tomato, for example, isoforms PSY1, CYCB and BCH2 (CRTR-B2) participate in carotenoid biosynthesis in chromoplasts whereas PSY2, LCYB and BCH1 (CRTR-B1) are found in chloroplasts [92]. In tomato chromoplasts PSY is active when soluble [93] and it associates with a multienzymatic complex with IPP/DMAPP isomerase (IDI) and GGPPS enzymes [94]. In chromoplasts of daffodil and cauliflower, PSY and PDS have been found in membrane-bound form and soluble in the stroma but only the membrane-associated enzymes are predicted to be active $[86,91]$. Other downstream enzymes of the pathway such as ZDS, LCYs, BCH, and PYP have been identified in the plastoglobule proteome from chromoplasts of different species, supporting the crucial role of these subcompartments in the channeling and production of carotenoids [43, $63,83,87,88,90]$.

\subsubsection{Storage in plastids}

The final accumulation of carotenoids in plastids not only depends on (1) the biosynthetic rate (e.g. the supply of MEP-derived precursors and the levels and activity of biosynthetic enzymes) but also relies on (2) the availability of plastidial structures for their sequestration and storage, and on (3) the carotenoid degradation rate. The presence of suborganellar membranous systems and other carotenoid-sequestering structures depends on the plastid type, whereas the predominant plastid type in a tissue determines the capacity of the tissue to produce and store carotenoids $[41,95,96]$. Meristematic tissues contain proplastids, which are undifferentiated plastids without the capability to synthesize carotenoids. Amyloplasts and elaioplats are specialized plastids from non-photosynthetic tissues (e.g. seeds, tubers or fruits) which store large quantities of starch and lipids, respectively, and contain variable amounts of carotenoids [97]. Etioplasts are characterized by the presence of prolamellar bodies [98]. Besides chlorophyll precursors, they contain low amounts of carotenoids, mainly violaxanthin and lutein, that contribute to greening when etioplasts differentiate into chloroplasts after exposure to light [99, 100]. Chloroplasts are the best studied plastids since they are the site of photosynthesis in green plant tissues. In chloroplasts, carotenoids are localized in the envelope, grana and thylakoid membranes, and reaction centers of photosystems [39]. The carotenoid composition of chloroplasts is quite similar in all plants. The predominant carotenoids in green tissues of plants grown under normal light conditions are lutein, $\beta$-carotene, violaxanthin, and neoxanthin, with lower amounts of zeaxanthin and $\alpha$-carotene. This profile can change in response to developmental (e.g. senescence) or environmental challenges. Notably, under bright light, the VAZ cycle leads to a strong increase in zeaxanthin and the $L X L$ cycle to higher levels of lutein to improve photoprotection (Figure 4).

In chloroplasts, only a reduced proportion of carotenoids are localized in plastoglobules. In other plastid types, however, plastoglobules can be the main structure for carotenoid deposition [83]. This is the case of some types of chromoplasts [95, 101, 102]. Chromoplasts are plastids specialized in carotenoid biosynthesis and storage. derive from other plastid types, including chloroplasts [101, 102]. According to the major internal substructures, chromoplasts can be classified as globular, tubular, crystalloid or membranous [95, 103]. This diversity reflects the wide variety of carotenoid profiles found in chromoplasts from different plant species, tissues, organs, or developmental stages. Interestingly, different chromoplast types and substructures can coexist in the same tissue $[101,104]$. A strong relationship between carotenoid deposition form and their bioavailability has been established, making this an attractive area of development for food research specialists [103] (see Section 4.3 below). Globular chromoplasts contain carotenoids in plastoglobules where more apolar carotenes or esterified xanthophylls are lipid-dissolved in the core of the globule and polar carotenoids are associated with the surrounding lipid monolayer [105]. Carotenoids stored in plastoglobules are very stable. In 
tubular chromoplasts, carotenoids are in a liquid crystalline state and sequestered in the core of a tube surrounded by a monolayer of glycolipids and phospholipids. Tubular structures are usually associated with plastoglobules in globular-tubular chromoplasts [103]. Crystalloid chromoplasts, typically found in tissues that accumulate large amounts of $\beta$-carotene or lycopene, contain solid carotenoid crystal structures surrounded by a lipid bilayer [106]. Membranous chromoplasts, identified only in few species and tissues, are characterized by the presence of concentric internal stacked membranes $[106,107]$. Recently, it has been shown that transgene-mediated overproduction of the DnaJ-like protein ORANGE (Or) induces the biogenesis of $\beta$-carotene-enriched membranous chromoplasts with higher carotenoid sink strength [100]. The Or protein was first identified as responsible for the orange color of the curd of the cauliflower Or mutant $[108,109]$. A specific role of Or in triggering chromoplast differentiation has been confirmed in different plant systems using transgenic approaches [100, 109-111]. However, the increase in carotenoid levels induced by Or overexpression in rice and maize seed endosperm does not correlate with the differentiation of proplastids into chromoplasts [112, 113]. Furthermore, Or may also promote carotenoid accumulation by increasing PSY activity [67, 68] and by inhibiting the conversion of $\beta$-carotene into downstream products [114]. Although a few other proteins (e.g. fibrillins) have been associated with increased levels of carotenoids in chromoplasts $[115,116]$, the Or protein is the only one known to have a major role in triggering the differentiation of chromoplasts.

\subsection{Carotenoids in non-plant systems}

\subsubsection{Archaea and non-photosynthetic bacteria}

In contrast to eukaryotes, that only produce $\mathrm{C} 40$ or C40-derived carotenoids, bacteria synthesize all $\mathrm{C} 45$ and most of the $\mathrm{C} 30$ and $\mathrm{C} 50$ carotenoids found to date, while the rest are produced by archaea. The Carotenoid DataBase [1] includes 307 carotenoids from 170 bacterial species and 19 carotenoids from 9 species of archaea. Another valuable tool dedicated to organize and classify the diverse carotenoids found in bacteria and archaea is the Prokaryotic Carotenoid DataBase, ProCarDB (http://bioinfo.imtech.res.in/servers/procardb/). ProCarDB [117] presently displays 289 carotenoids distributed in acyclic (40), cyclic (31), hydroxycarotenoids (also known as carotenols) (75), and hydroxycarotenoids with cyclic structures (143). In addition, ProCarDB assigns the different carotenoids to 48 pathways.

Although carotenoid biosynthesis is less common in archaea, it has been described in Halobacterium salinarum, Halorubrum chaoviator, Halococcus morrhuae, Natromonas pharaonis and Haloarcula japonica. Recently, other carotenoid-producing haloarchaea such as Haloferax volcanii and Haloferax mediterranei were characterized in salty ponds or marshes $[118,119]$. The main final product of the carotenoid pathway in archea is the acyclic polyhydroxylated C50 carotenoid bacterioruberin $[120,121]$ (Figure 5). For instance, in H. salinarum 5-cis, 9-cis, 13-cis, and all-trans-bacterioruberin isomers account for $13 \%, 4 \%, 11 \%$, and $68 \%$, respectively, of all carotenoids, whereas all-trans isomers of trisanhydrobacterioruberin and bisanhydrobacterioruberin correspond to $4 \%$ and $1 \%$, respectively [121]. Bacterioruberin is not exclusive of archaea, as it has also been found in bacteria such as Azospirillum brasilensis, Micrococcus roseus, Rubrobacter radiotolerans, Arthrobacter agilis, Kocuria rosea or Thermus filiformis [121, 122].

While archaea use the MVA pathway to supply the isoprenoid precursors necessary to synthesize their carotenoids, most bacteria use the MEP pathway. A minority of bacteria use the MVA pathway, and only a few of them use both pathways [123]. Phylogenetic analysis, however, suggests that the MEP pathway is the ancestral route in bacteria. In the case of nonphotosynthetic bacteria, most use IPP and DMAPP to produce C20 GGPP and downstream C40 carotenoids. Representative examples are Erwinia sp., Myxococcus sp., Streptomyces sp., Alcaligenes sp., Flavobacterium sp., Agrobacterium aurantiacum (Paracoccus sp.) and Thermus thermophilus. Erwinia species accumulate $\beta$-cryptoxanthin, zeaxanthin, and glucosides of these xanthophylls [124]. Myxococcus fulvus produces 4-ketotorulene and some unique C40 
carotenoids, such as myxobactin and myxobactone esters (Figure 5) [125, 126]. By contrast, Clostridia and Bacilli, such as Staphylococcus aureus, Streptococcus faecium, Heliobacillus fasciatum, and Heliobacterium, produce C30 carotenoids through the diapocarotene or C30 bacterial pathway (Figure 5), which uses C15 farnesyl diphosphate (FPP) as the prenyl diphosphate precursor instead of GGPP [127]. This pathway produces different acyclic compounds derived from 4,4'-diapolycopene or 4,4'-diaponeurosporene, such as $\mathrm{OH}$ diaponeurosporene glucoside ester or 4,4'-diapolycopene-4,4'-dioic acid diester (Figure 5).

The identification of the first prokaryotic genes for carotenoid biosynthesis settled a terminology (crt) that has been maintained to refer to homologous genes in many other bacteria (Table 1). The genes encoding GGPP synthase (CrtE), phytoene synthase (CrtB), phytoene and $\zeta$ carotene desaturases ( $\mathrm{Crtl}$ and $\mathrm{CrtQ}$ ), and lycopene cyclase (CrtY) are conserved between nonphotosynthetic and anoxygenic photosynthetic bacteria, and similar genes are also found in algae and plants. Hydroxylations and glycosylations are carried out by CrtZ and CrtX, respectively. Enzymatic activities of the Erwinia enzymes were demonstrated by heterologous expression of one or several crt genes in engineered strains of Escherichia coli, a non-carotenogenic host [128]. This experimental system has actually allowed biochemical assignments for many carotenoid biosynthetic genes from both prokaryotic and eukaryotic organisms. Genes for bacterial carotenoid biosynthesis and regulation are usually organized as operons. Streptomyces coelicolor has two crt operons, crtEIBV and crtYTU [129]. In Flavobacterium sp, the genomic orientation of the crt cluster is different to other bacteria, as crtE gene is transcribed oppositely to the crtZYIB operon [130]. In some species, the crt genes are extrachromosomal. This is the case of Thermus thermophiles, where the crt operons are located in a 260-kb circular plasmid named pTT27 [129].

Carotenoid biosynthesis is regulated by different environmental stimuli, being light the most studied. Light promotes the synthesis of carotenoids in Myxococcus xanthus, S. coelicolor and $T$. thermophilus, among others [129]. This photoregulatory mechanism has been studied in detail in $M$. xanthus, where light is detected by the blue-light receptor CarF through the formation of singlet oxygen $\left({ }^{1} \mathrm{O}_{2}\right)$ in the membrane [131]. Blue light excites protoporphyirin IX (PPIX) to ${ }^{3} \mathrm{PPIX}$ and this converts $\mathrm{O}_{2}$ in ${ }^{1} \mathrm{O}_{2}$. The singlet oxygen is detected by CarF, which in turn interacts with CarR to inactivate it [132]. CarR is an anti- $\sigma$ factor that sequesters $\sigma$ factor CarQ in the dark. In the presence of blue light, CarQ is free to bind to the core of RNA polymerase and to activate $\mathrm{P}_{\mathrm{QRS}}$ and $\mathrm{P}_{1}$ promoters of the regulatory carQRS operon and the crtlb gene. Both promoters also need the regulatory complex CarD/CarG for their transcription. The rest of the genes involved in carotenoid biosynthesis are in two linked operons: carB (encoding carotenogenic genes $c r t E-l a-B-D-C)$ and carA ( $c r t Y c-Y d$-carA-carH). CarA and CarH are DNAbinding repressors that are counteracted by CarS, that is induced by light from the carQRS operon [131].

In S. coelicolor, T. thermophilus and Bacillus megaterium, the MerR family transcriptional regulator LitR is very well conserved and uses adenosyl B12 as a light sensing ligand. In the dark, it behaves as a repressor of a sigma factor (LitS) but in the light LitR activates the transcription of litS and the carotenoid biosynthesis genes [129]. This regulation by light offers some clues on possible functions of carotenoids in non-photosynthetic prokaryotes. Carotenoids most likely act as antioxidants in non-photosynthetic bacteria, protecting against photooxidative protoporphyrin and heme molecules [133]. Carotenoid extracts of Deinococcus radiodurans, a radio-resistant species, protect against DNA-damaging agents $[134,135]$. In the human pathogen $S$. aureus, staphyloxanthin contributes to the resistance to reactive oxygen species (ROS) and to the attack of neutrophils, being the role of this carotenoid to resist the host phagocytic defenses $[136,137]$.

\subsubsection{Photosynthetic bacteria}

Anoxygenic photosynthetic bacteria producing carotenoids include purple bacteria, green sulfur bacteria, and heliobacteria. Genes involved in carotenoid biosynthesis in these bacteria are listed in Table 1. The structures of the produced carotenoids differ from those of other 
organisms, including non-photosynthetic bacteria. Most $\mathrm{C} 40$ carotenoids from these bacteria are acyclic and contain sulfate, methoxy, carbonyl, carboxyl, glycoside, and hydroxyl groups [127]. Five main carotenogenesis pathways have been proposed for anaerobic photosynthetic bacteria (Figure 5): (1) the spirilloxanthin pathway, that is divided in normal spirilloxanthin, unusual spirilloxanthin, spheroidene, and carotenal pathways. As an example, the purple bacteria Rhodospirillum rubrum, Rhodobacter capsulatus and Rhodobacter sphaeroides produce spirilloxanthin and spheroidene [138]; (2) the isorenieratene pathway, that also includes that producing chlorobactene. Purple and green sulfur bacteria such as Chlorobium tepidum and Chlorobium phaeobacteroides produce $\mathrm{C} 40$ carotenoids with aromatic rings such as isorenieratene and chlorobactene, the latter also present in actinobacteria; (3) the okenone pathway, that includes the okenone and the R.g-keto carotenoid pathways; (4) the $\gamma$ - and $\beta$ carotene pathway; and (5) the diapocarotene pathway, which in heliobacteria such as Heliobacillus mobilis, Heliophilum fasciatum, and Heliobacterium chlorum produces diapolycopene. There are additional pathways leading to carotenoid glucosides and acylated carotenoid glucosides (Figure 5). In the case of aerobic photosynthetic bacteria, most of them use the spirilloxanthin pathway [139].

Similar to plants, the main function of carotenoids in both anoxygenic and oxygenic photosynthetic bacteria is to protect from oxidative damage provoked by excess light in the presence of excited bacteriochlorophyll and oxygen. Carotenoids also have a light-harvesting function, as they absorb light and transfer it to the photosynthetic reaction center via bacteriochlophyll $[133,140]$. In cyanobacteria, the oldest aerobic photosynthetic organisms and the origin of plant chloroplasts, carotenoids are also associated to photosynthesis and photoprotection, including the formation of diverse carotenoid-protein complexes [141, 142]. A well-known example is the Orange Carotenoid Protein, which contains a ketocarotenoid as photoresponsive chromophore. Absorption of blue-green light by the chromophore causes a structural change of the protein that triggers a photoprotective mechanism, decreasing the excess absorbed energy arriving at the photosynthetic reaction centers by increasing thermal dissipation at phycobilisomes [143].

Early studies identified $\beta$-carotene as one of the major carotenoids in cyanobacteria [144], accompanied by oxidized derivatives such as zeaxanthin, canthaxanthin, $\beta$-cryptoxanthin, and echinenone [145]. Genes homologous to those responsible for carotenoid biosynthesis in nonphotosynthetic bacteria are usually found in cyanobacteria, including those for the early steps ( $c r t E, c r t B, c r t l, c r t Q$ and $c r t L)$ and for downstream branches involving $\beta$-carotene hydroxylase $(c r t R)$ and ketolase (crtO/crtW) genes (Table 1). Some cyanobacteria contain plant-type desaturase genes, as crtP instead of crtl for phytoene desaturation. Strikingly, cyanobacteria lack carotenoids that are abundant in eukaryotic photosynthetic species, such as lutein, violaxanthin or neoxanthin, and synthesize carotenoids not found in other taxa. These unique carotenoids include the zeaxanthin derivatives caloxanthin and nostoxanthin (with two hydroxyl groups in the

$\beta$ ring resulting from the activity of the $c r t G$ gene in Synecococcus $s p$.) and glycoside derivatives such as the myxol glycosides and oscillol diglycosides [146]. Only a gene involved in glycosylation, $w c a G$, has been identified so far [146]. Carotenoid genes are induced by light and their regulation is mediated by the transcription factor NtcA, which binds to the crt promoters in the presence of reductants and oxoglutarate [147].

\subsubsection{Microalgae}

Microalgae are a heterogeneous group of photosynthetic unicellular organisms living in water ecosystems. In contrast to fungi, microalgae exhibit a complex carotenoid metabolism $[148,149]$. Similar to higher plants, they accumulate carotenoids involved in photosynthetic functions, e.g. photoprotectants and accessory light-absorbing pigments able to transfer energy to chlorophylls. Thus, microalgae frequently contain $\beta$-carotene, violaxanthin, antheraxanthin, zeaxanthin, neoxanthin, and lutein [150]. Some carotenoids, however, are specific of certain 
microalgae groups. The neoxanthin-related xanthophylls diatoxanthin, dinoxanthin, diadinoxanthin, and fucoxanthin are only found in diatoms such as those of the genus Phaeodactylum, while $\alpha$-carotene, $\varepsilon$-carotene, $\delta$-carotene or astaxanthin are only found in green algae (Figure 7) [148, 151]. Furthermore, stressing conditions such as nitrogen deprivation, strong solar irradiation, drought or hypersalinity cause some microalgae to accumulate large amounts of carotenoids that provide an intense reddish pigmentation to the stressed cells [152]. In this case, however, the carotenoids are not accumulated in the thylakoid membranes but in oily droplets or lipid vesicles located either in chloroplasts (in thylakoids) or in the cytoplasm (as oleosomes). To facilitate this, the biosynthesis of carotenoids in response to stress is metabolically coupled to a massive production of lipids $[152,153]$. Well-known examples are the accumulation of $\beta$ carotene by Dunaliella salina, astaxanthin by Haematococcus pluvialis, and lutein by Muriellopsis sp. and Scenedesmus almeriensis $[154,155]$. The large production of $\beta$-carotene in $D$. salina under high solar irradiation, which reaches up to $10 \%$ dry weight [156], occurs in vegetative cells [157]. In $H$. pluvialis, however, green vegetative cells growing under favorable conditions produce mainly lutein and $\beta$-carotene whereas exposure to stressing conditions results in the cessation of cell division and their transformation into cyst cells (called aplanospores) that accumulate astaxanthin up to $4 \%$ of the total dry mass [158]. To facilitate storage, astaxanthin is mostly accumulated in the form of fatty acid esters.

\subsubsection{Fung}

Carotenoid biosynthesis is a frequent trait in fungi, where the carotenoid pathways are simpler in comparison with photosynthetic organisms [159, 160]. In contrast to other carotenogenic organisms, fungal phytoene synthase and lycopene/carotene cyclase activities are encoded by a single gene, resulting in a bifunctional enzyme with amino and carboxyl domains similar to CrtY and CrtB, respectively. Fungi also harbor a single desaturase that achieves all the desaturation steps downstream of phytoene (either four or five, depending of the species). The production of $\beta$-carotene is predominant in the mucormycotina group, with Phycomyces blakesleeanus, Mucor circinelloides and Blakeslea trispora as the best-known examples. Only two genes (one encoding the phytoene synthase/cyclase and the other the desaturase), usually divergently transcribed from a shared regulatory region, are needed for $\beta$-carotene synthesis in these fungi. Accumulation of $\beta$-carotene has also been described in fungi from other taxonomic groups, such as basidiomycotina (e.g. Ustilago maydis) [161], and ascomycotina (e.g., Cercospora nicotianae) [162].

Other fungi produce xanthophylls (Figure 6). The most thoroughly investigated systems are those for the synthesis of neurosporaxanthin by the ascomycotina Neurospora crassa and Fusarium fujikuroi, and the production of astaxanthin and torularhodin by the basidiomycotina yeasts Xanthophyllomyces dendrorhous and Rhodotorula sp, respectively [160]. In the first case, five desaturations, a single cyclization and an oxidative cleavage reaction are needed to generate neurosporaxanthin. Astaxanthin biosynthesis requires the introduction of keto and hydroxyl groups in the terminal rings of $\beta$-carotene (Figure 6). All the genes participating in neurosporaxanthin production in $N$. crassa and Fusarium sp, and astaxanthin production in $X$. dendrorhous have been identified [160]. The genes up to torulene biosynthesis were similar to those from other biosynthetic pathways, but the last two steps of neurosporaxanthin production were found to be carried out by two unique enzymes, a torulene-cleaving dioxygenase (a novel member of the carotenoid cleavage dioxygenase or CCD family, see Section 2.3 below) that produces $\beta$-apo-4'-carotenal $[163,164]$ and an aldehyde dehydrogenase that generates the terminal carboxy group $[165,166]$. Less information is available on the genetics and biochemistry of torularhodin biosynthesis, which is similar to that of neurosporaxanthin but lacks a cleavage step (Figure 6). In the case of Fusarium, a side branch of neurosporaxanthin biosynthesis leads to $\beta$-carotene, which can be then cleaved by another CCD enzyme to produce retinal, presumably used as chromophore by the Fusarium opsins [167]. The amenability of fungi to genetic 
manipulation and industrial cultivation makes some of them ideal choices for biotechnological carotenoid production, as that of $\beta$-carotene or lycopene by $B$. trispora, or astaxanthin by $X$. dendrorhous [168]. The availability of genetic engineering tools for $X$. dendrorhous has allowed its use for the production of alternative xanthophylls such as zeaxanthin [169] or new ketocarotenoids [170].

In contrast to photosynthetic species, carotenoids appear to play only secondary roles in fungi. Loss of carotenoids in albino mutants of carotenogenic fungi does not have an impact on growth under laboratory conditions, whereas well-known fungal models such as the yeast Saccharomyces cerevisiae or the mold Aspergillus nidulans completely lack carotenoids. However, different lines of evidence suggest that carotenoids might exert protective roles against oxidative stress [171]. They also serve as precursors for bioactive molecules such as retinal, as indicated above. However, the incapacity to produce retinal has no significant consequences in F. fujikuroi, and mutants unable to produce CarO or OpsA rhodopsins have no apparent phenotypic alterations $[172,173]$. In some mucormycotina, $\beta$-carotene is also used as precursor in the synthesis of sexual hormones, known as trisporic acids, as well as other compounds [171]. The incapacity to produce trisporic acids in $\beta$-carotene defective mutants of $P$. blakesleeanus results in an impairment to carry out the sexual cycle [174].

\subsubsection{Animals}

Animals were long believed to be incapable of synthesizing carotenoids de novo despite requiring them for communicative coloration and to serve as precursors for the production of retinoids (including vitamin A, biologically active molecules such as retinoic acid, and vision pigments). Carotenoids are actually taken from the diet or through symbiosis in most animals [175]. However, the previously assumed dogma that animals do not synthesize carotenoids has been challenged by the recent discovery of genes encoding carotenoid biosynthetic enzymes of fungal origin (bifunctional phytoene synthase/carotene cyclases and desaturases) in the genomes of arthropods such as hemipteran (aphids, adelgids, phylloxerids) and dipteran (gall midges) insects and mites [176-181]. The presence of these enzymes allows the endogenous production of carotenoids such as $\alpha$-carotene, $\gamma$-carotene, $\beta$-carotene, torulene, dehydro- $\gamma, \psi$-carotene (a carotenoid similar to torulene) and ketocarotenoids in the animal hosts. The corresponding biosynthetic genes were likely incorporated to these phylogenetically distant arthropod genomes in different horizontal gene transfer events. Their presence appears to be of ecological significance because it determines color polymorphism and hence influences susceptibility to natural enemies [176]. Next-generation sequencing technologies should facilitate the discovery of more examples of transference of carotenoid-related genes from microbial to animal genomes.

Animals that do not produce carotenoids de novo can instead accumulate them in particular tissues. Mammals have been traditionally divided into "white-fat" (pig, sheep, goat, carnivores and rodents) or "yellow-fat" (cattle, horses) animals according to their ability to accumulate carotenoids in their adipose tissue. Those in the first group hardly absorb carotenoids whereas the animals of the second group mainly absorb carotenes [182]. Ruminants accumulate $\beta$-carotene in the milk that is then transferred to dairy products [183]. Interestingly, both carotenes and xanthophylls (e.g. lutein) can be found in the human milk [184], but the possible beneficial roles of lutein in our early life are only beginning to be investigated [185]. Other animals such as birds or amphibians absorb mainly xanthophylls [182]. For example, lutein and zeaxanthin are commonly found in egg yolks from hens [186]. Birds also accumulate carotenoids in their plumage as a social interaction display [187]. The distinctive colors of many edible fish (salmon, trout), mollusks (mussel, clam), and crustaceans (shrimp, lobster) are also due to carotenoids (in some cases found as carotenoproteins) [188]. Carotenoids with diverse chemical structure (lutein, zeaxanthin, $\beta$-cryptoxanthin, canthaxanthin, astaxanthin, citranaxanthin capsanthin, $\beta$-apo-8'-carotenal, $\beta$-apo-8'-carotenoic acid ethyl ester) are widely used in animal feeds for pigmentation of dairy products, egg yolk, and several aquaculture fish $[29,188,189]$. 


\subsection{Carotenoid cleavage products}

Virtually all living organisms are able to catabolize carotenoids not only by non-enzymatic and unspecific reactions such as lipoxygenase cooxidation or photooxidation [190] but also by enzymatic oxidative breakdown $[191,192]$. It is important to note that, despite the term "apocarotenoid" is often used to refer to all carotenoid cleavage products, only a fraction of these degradation products are actual apocarotenoids. For consistency, we will use the acronym CCPs for carotenoid cleavage products in general and only use the term apocarotenoids for carotenoid products that lost fragments at one or both ends of the molecule. In plants, CCPs can undergo further modifications (such as oxydations, reductions, cyclizations, methylations, or glycosylations) to render biologically active molecules. In many cases, cleavage of the carotenoid molecules results in volatile products or new water-soluble pigments. CCPs can also act as defense compounds against herbivores and pathogens, e.g. repellents or chemoattractants, as signals for predation and seed dispersal, as allelochemicals providing competitive advantages, as mediators of symbiotic associations, and as phytohormones, e.g. ABA and strigolactones [40, 190, 193-199]. In addition, unknown carotenoid-derived molecules appear to regulate different developmental processes in plants [40, 198]. For example, the BYPASS signal is an uncharacterized graft-transmissible metabolite that functions as a negative regulator of both root and shoot growth [200, 201]. Root and leaf development are also controlled by unknown compounds derived from carotenoids [202, 203]. Plant CCPs also have an important impact in human health. The health promoting properties of apocarotenoids from saffron (Crocus sativus) and bixa (Bixa orellana) have been known for a long time [204, 205]. Several reports have shown that $A B A$ is present and functionally active in humans [206], whereas strigolactones may have anti-cancer activity [207, 208]. Screening of aroma compounds revealed a cancer chemopreventive potential for volative CCPs such as $\beta$-ionone and members of the damascone group [209].

The enzymes catalyzing the oxidative cleavage of carotenoids to form CCPs are known as carotenoid cleavage dioxygenases (CCDs). These enzymes, which also recognize and cleave CCPs, are non-heme iron-containing enzymes with a common basic architecture consisting of a rigid seven-bladed $\beta$-propeller which forms a long tunnel on the central axis of the protein where the reaction center is located [210]. CCDs normally catalyze the cleavage of non-aromatic double bonds in the polyene chain by oxygen $\left(\mathrm{O}_{2}\right)$ to yield aldehyde or ketone compounds $[211,212]$. They are typically encoded by multigene families in bacteria, fungi, plants and animals [213]. The first CCD was identified in the ABA-deficient maize mutant $V p 14$ [214] and named as 9-cisepoxycarotenoid dioxygenase (NCED) [215]. Since then, homology-based searches have led to the isolation and characterization of many other enzymes involved in CCP biosynthesis $[40,213]$. Two main subfamilies of CCDs are found in plants: NCEDs (exclusively involved in ABA biosynthesis and localized in plastids) and CCDs (not involved in ABA production). The CCD enzymes (CCD1, CCD2, CCD4, CCD7 and CCD8) differ in their subcellular location, regulation, substrate specificity and cleavage activity. As a consequence, despite starting from a limited number of carotenoid substrates they give rise to a vast range of CCPs with diverse functions (including hormones, signals, and volatile/flavor compounds).

The sites recognized and cleaved by particular CCD enzymes are shown in Figure 8. CCD7 and CCD8 act sequentially for the production of the strigolactone precursor carlactone [199, $216]$. The initial substrate is 9-cis- $\beta$-carotene, synthesized from $\beta$-carotene by the carotenoid isomerase DWARF27 [216-218]. The Arabidopsis thaliana CCD1 enzyme was initially found to act on a variety of carotenoids at their 9-10 and $9^{\prime}-10^{\prime}$ double bonds [219], although further studies showed a relaxed double bond specificity [220]. In maize, CCD1 transcript abundance negatively correlates with carotenoid content in the grain endosperm [221-223]. By contrast, manipulation of CCD1 activity alters CCP levels [224] but not the carotenoid content of the other plant tissues $[220,225,226]$. These features, together with the cytosolic localization of the enzyme, led to suggest that CCD1 enzymes might not cleave intact carotenoids but act as scavengers of mixtures 
of CCPs of different chain lengths and generate new bioactive CCPs with yet unknown functions. Closely related to CCD1, the plastid-localized CCD2 enzymes have only been found in Crocus species $[227,228]$. CCD2 catalyzes the cleavage of zeaxanthin to produce crocetin and 4-hydroxy2,6,6-trimethyl-1-cyclohexene-1-carboxaldehyde. These two CCPs likely act as signals to attract pollinators and seeds dispersers [229] but they also display important pharmacological properties [204]. The CCD4 subfamily, the largest CCD subfamily in plants [213], shows a diversity of cleavage reactions and expression patterns [229-234], including the same enzymatic activity displayed by CCD2 in Crocus species [235]. Thus, CCD4 activity has been associated with the production of flavor volatiles and colored CCPs but also with carotenoid recycling during leaf senescence and seed desiccation [236], stolon development [237], and chloroplast biogenesis [202].

\section{Crop biotechnology}

Over the last three decades, many genes involved in carotenoid biosynthesis, storage and degradation have been cloned and some of them used to alter the carotenoid contents of crops. Carotenoid engineering strategies involve direct interventions to boost the accumulation of target carotenoids by (1) increasing flux through the pathway via overexpression of rate-limiting enzymes to relieve metabolic bottlenecks; (2) suppressing competing pathways downstream of branch points to avoid intermediates being diverted; (3) creating carotenoid sequestering structures and improving plastid sink capacity to store carotenoids; or (4) suppressing catabolic steps so that target carotenoids are not degraded or converted into undesired CCPs. Carotenoid engineering can also be used to produce carotenoids that are not naturally made in crops because the corresponding metabolic steps are missing. These include commercially important ketocarotenoids such as astaxanthin (Figures 5 and 6) and canthaxanthin (Figure 2). In this section we will briefly review major progresses in the metabolic engineering of carotenoids in different crops. The development of cultivars with elevated carotenoid content using classical breeding has been extensively reviewed elsewhere $[238,239]$ and will not be covered here.

\subsection{Leaves and roots}

\subsubsection{Tobacco (Nicotiana tabacum) and related species}

Since the demonstration that plant leaves could be used to produce non-native ketocarotenoid pigments such as astaxanthin [240-242], many efforts have been focused on diverting the endogenous carotenoid pathway of several plants towards the biosynthesis of ketocarotenoids in their leaves. Perhaps the most outstanding examples include the generation of transplastomic lines and nuclear transformants of $N$. tabacum and the nicotine-free tobacco tree species $N$. glauca [243-245]. Transplastomic tobacco ( $N$. tabacum) plants harboring codonoptimized genes from the marine bacterium Brevundimonas encoding $\beta$-carotene ketolase (crtW) and $\beta$-carotene hydroxylase ( $c r t Z$ ) produced up to $5 \mathrm{mg} / \mathrm{g}$ DW of astaxanthin, reaching more than $0.5 \%$ dry weight [243]. More than $70 \%$ of total carotenoids were transformed into ketocarotenoids in leaves, turning their green color into reddish-brown [243]. The same two genes have also been overexpressed in transgenic potato [246] and $N$. glauca [245] via Agrobacterium-mediated transformation. However, ketocarotenoid levels present in these lines were not as high as those found with transplastomic tobacco ( $N$. tabacum) lines [243]. Using a similar strategy, a synthetic operon consisting of the Brevundimonas crtW and crtZ genes together with a plant LCYB-encoding gene was inserted into the tobacco ( $N$. tabacum) chloroplast genome [244]. Astaxanthin accumulation in young leaves reached $10 \mathrm{mg} / \mathrm{g}$ DW (i.e. $1 \%$ of the dry weight of the plant), levels that are 2 -fold higher than those maximally achieved in the previous attempt [243]. Most interestingly, the transformed chloroplast genome was then allowed to move from $N$. tabacum into $N$. glauca by grafting, a procedure that facilitates horizontal genome transfer between plants and thus provides a straightforward method for extension of the transplastomic technology to new species. The pigment profiles in leaves of transplastomic $N$. 
glauca trees expressing the synthetic pathway were very similar to those found in $N$. tabacum [244]. In N. glauca flowers, which are normally yellow due to the accumulation of carotenoids such as lutein $[241,242]$, expression of the astaxanthin operon resulted in deep-orange petals, indicating conversion of the floral carotenoid pool. Transplastomic N. glauca flowers, however, still accumulated significant amounts of non-astaxanthin carotenoids, most likely because of the much lower activity of plastid gene expression in non-green tissues [244].

\subsubsection{Lettuce (Lactuca sativa)}

Lettuce is a vegetable whose leaves synthesize a rare $\varepsilon, \varepsilon$-carotenoid, lactucaxanthin. With the goal of producing novel ketocarotenoids in the leaves, transplastomic lettuce plants were generated that expressed three codon-optimized genes from marine bacteria: $c r t W$ and $c r t Z$ from Brevundimonas $s p$. and IDI from Paracoccus $s p$. [247]. The transplastomic plants mainly produced astaxanthin fatty acid esters (67.4\% of total carotenoids), free astaxanthin $(10.0 \%)$ and other ketocarotenoids $(17.5 \%)$. The artificial ketocarotenoids corresponded to $94.5 \%$ of total carotenoids [247].

\subsubsection{Carrot (Daucus carota)}

Carrot roots provide an important source of dietary $\beta$-carotene and $\alpha$-carotene. Promoting the metabolic flux to carotenoids by overexpressing genes encoding DXS [248] or PSY [249] successfully increased the production of carotenoids in transgenic roots. Both total carotenoid and $\beta$-carotene levels also increased in transgenic carrots constitutively overexpressing the endogenous $\angle C Y B 1$ gene [250]. In this case, transgenic lines showed higher levels of the endogenous PSY2 gene (but not the PSY1 gene), which probably contributed to raising the total carotenoid level in roots [250]. Constitutive overexpression of the carrot LCYB1 gene in tobacco ( $N$. tabaccum) resulted in increments in $\beta$-carotene and total carotenoids, increased expression of endogenous DXS2, PSY1 and PSY2 genes, and, surprisingly, a concomitant increase in plant height and biomass [251]. Changes in the expression of endogenous carotenoid-related genes were also observed in transgenic carrot plants overexpressing genes encoding Arabidopsis DXS [248] or $\mathrm{H}$. pluvialis $\beta$-carotene ketolase [252]. In the latter, up to $70 \%$ of total carotenoids were converted to novel ketocarotenoids, reaching up to $2400 \mu \mathrm{g} / \mathrm{g} \mathrm{DW}$ in the root. Astaxanthin, adonirubin, and canthaxanthin were most prevalent, followed by echinenone, adonixanthin and $\beta$-cryptoxanthin [252].

\subsection{Fruits and tubers}

\subsubsection{Tomato (Solanum lycopersicon)}

Tomato products are the principal dietary sources of lycopene and a major source of $\beta$ carotene. As PSY activity is crucial to control the carotenoid pathway during tomato fruit ripening, it was early selected as a prominent target for the genetic engineering of the carotenoid content of tomato fruit. The first constitutive expression of a PSY-encoding gene in tomato resulted in carotenoid-rich seed coats, cotyledons and hypocotyls [253]. However, transgenic plants showed a dwarf phenotype because GGPP was diverted to the carotenoid pathway and hence became less available for the synthesis of the growth-promoting hormones gibberellins [253] (Figure 4). This work illustrated how problems arise when a balanced metabolism is disturbed. By using fruit-specific promoters, many of the problems associated with PSY overproduction in vegetative parts of the plant were circumvented. For example, fruit-specific expression of a bacterial PSY-encoding gene $(\operatorname{crtB})$ resulted in up to 4-fold higher fruit carotenoids [61]. Upstream MEP pathway precursors are also rate limiting in tomato [254], so increasing the precursor supply can direct additional flux into the pathway thus increasing total carotenoid levels. This strategy was used in tomato, where the overexpression of the Escherichia coli gene encoding DXS resulted in a 1.6-fold increase in total fruit carotenoids [255]. 
Manipulation of the desaturation activity in tomato was achieved with a bacterial crtl gene encoding phytoene desaturase/isomerase [256]. While expression of this gene did not elevate total carotenoid levels in transgenic tomatoes, the $\beta$-carotene content increased about 3 -fold to reach $45 \%$ of the total carotenoid content. Unlike PSY, which was down-regulated, the activity of endogenous carotenoid biosynthetic enzyme (PDS, ZDS, LCYB) was concurrently increased via upregulation of the expression of the corresponding genes. Similarly, expression of $\mathrm{crtl}$ in the tomato mutants tangerine (defective in CRTISO) and old gold crimson $\left(\mathrm{og}^{c}\right.$, defective in CYCB, the ripening-specific LCYB isoform) reduced the total carotenoid content in tomato fruits due to the downregulation of PSY activity [257]. Transgene expression resulted in the induction of CYCB in the tangerine mutant and $\angle C Y B$ in the $o g^{c}$ mutant [257], similar to a previous report [256]. Thus, transgenic tomato lines expressing crtl exhibited negative feedback mechanisms acting predominantly at the level of PSY and feed-forward mechanisms inducing cyclization [257]. Overexpression of a tomato $L C Y B$ gene resulted in high amounts of $\beta$-carotene (up to $205 \mu \mathrm{g} / \mathrm{g}$ $\mathrm{FW}$ ) in the transgenic ripe fruits as a result of the almost complete cyclization of lycopene [258]. However, elevating $\beta$-carotene levels without reducing lycopene levels is more interesting for plant breeders. Two recent works have achieved this goal, resulting in tomato ripe fruits that were orange instead of red when ripe. In one of them, expression of a $N$. tabacum gene encoding LCYB driven by the Arabidopsis UBQ3 promoter resulted in enhanced levels of $\beta$-carotene in tomato fruit (up to 3 -fold, $783 \mu \mathrm{g} / \mathrm{g} \mathrm{DW}$ ) with negligible changes of other carotenoids, including lycopene [259]. The second work investigated the activity of the fruit Clp protease during ripening [66]. Down-regulation of this protease disrupted normal chromoplasts differentiation and caused an increased accumulation of DXS and PSY enzymes but also of the Or chaperone, eventually resulting in ripe fruits with 2 -fold higher levels of $\beta$-carotene but normal levels of lycopene [66].

Tomato fruit is an excellent source to overproduce astaxanthin because sufficient precursors (lycopene and $\beta$-carotene) are available and the storage capacity for lipophilic carotenoids is high [260]. This was further improved by constitutive co-expression of Chlamydomonas reinhardtii $\beta$-carotene ketolase and Haematococcus pluvialis $\beta$-carotene hydroxylase, a unique pair of enzymes identified to co-operate in converting $\beta$-carotene to astaxanthin. Expression of the two enzymes in tomato up-regulated most intrinsic carotenogenic genes, and efficiently directed carbon flux into carotenoids, leading to massive accumulation of mostly free astaxanthin in leaves $(3.12 \mathrm{mg} / \mathrm{g} \mathrm{DW})$ but esterified astaxanthin in fruits $(16.1 \mathrm{mg} / \mathrm{g}$ DW) and a 16-fold increase of total carotenoid capacity therein without affecting plant growth and development [261]. This study opened up the possibility of employing crop plants as green factories for economical production of astaxanthin. More recently, ketocarotenoids were produced at high levels $(3.0 \mathrm{mg} / \mathrm{g} \mathrm{DW}$ ) in tomato fruit expressing the Brevundimonas genes CrtZ and $\mathrm{Cr} W \mathrm{~W}$ followed by $\beta$-carotene enhancement through the introgression of a CYCB-encoding allele from a Solanum galapagense background [262]. The predominant carotenoids were fatty acid esters of phoenicoxanthin, and scalability studies showed potential to deliver $23 \mathrm{~kg}$ of ketocarotenoid per hectare. Most interestingly, aquaculture trials demonstrated that the plantbased feeds developed from these lines were more efficient than the synthetic feed to color trout flesh, showing up to 2 -fold higher retention of the main ketocarotenoids in the fish fillets [262].

\subsubsection{Orange (Citrus sinensis)}

Orange is a major crop and an important source of health-promoting bioactive compounds. Orange fruits accumulate a high amount of xanthophylls, especially $\beta, \beta$-xanthophylls. Through blocking the expression of an endogenous $\mathrm{BCH}$-encoding gene involved in the conversion of $\beta$ carotene into xanthophylls, significant increase of $\beta$-carotene levels (up to 36 -fold) were achieved in the pulp of sweet orange fruit [263]. 


\subsubsection{Potato (Solanum tuberosum)}

Tubers are underground storage organs that can derive from stems (e.g. potato and yam) or roots (e.g. sweet potato and cassava). Potato tubers overexpressing a bacterial crtB gene accumulated total carotenoid levels up to $78 \mu \mathrm{g} / \mathrm{g}$ DW and $\beta$-carotene up to $6.5 \mu \mathrm{g} / \mathrm{g}$ DW [264]. Overexpression of a bacterial DXS-encoding gene resulted in total carotenoid levels of $6.8 \mu \mathrm{g} / \mathrm{g}$ DW, a 2-fold increase compared to untransformed controls [265], whereas co-expression of bacterial $c r t B, c r t l$, and $c r t Y$ resulted in a significant enhancement of total carotenoid levels up to $114 \mu \mathrm{g} / \mathrm{g}$ DW and $\beta$-carotene levels up to $47 \mu \mathrm{g} / \mathrm{g}$ DW [266]. Silencing of the endogenous LCYE gene by expressing an antisense RNA construct led to 14 -fold more $\beta$-carotene $(2.6 \mu \mathrm{g} / \mathrm{g} \mathrm{DW}$ ) compared to untransformed tubers, whereas total tuber carotenoids increased up to 2.5 -fold (8.7 $\mu \mathrm{g} / \mathrm{g}$ DW) [267]. Another common target of specific enzyme inhibition is $\mathrm{BCH}$, as this would prevent the conversion of $\beta$-carotene into zeaxanthin (Figure 4) and therefore force the accumulation of $\beta$-carotene as an end-product. Silencing of the corresponding potato gene increased $\beta$-carotene levels to $16.55 \mu \mathrm{g} / \mathrm{g}$ DW (a 331-fold improvement) and lutein to $6.85 \mu \mathrm{g} / \mathrm{g}$ DW (a 2.5-fold improvement), while zeaxanthin levels were reduced from 29 to $6 \mu \mathrm{g} / \mathrm{g} \mathrm{DW}$, a five-fold reduction [268]. In another successful example, the zeaxanthin content of potato tubers was enhanced by silencing the endogenous gene encoding ZEP [269]. This increased the total carotenoid levels by 5.7 -fold $(60.8 \mu \mathrm{g} / \mathrm{g} \mathrm{DW}), \beta$-carotene levels by 3.4 -fold $(2.4 \mu \mathrm{g} / \mathrm{g} \mathrm{DW})$, and zeaxanthin levels by 133 -fold $(40 \mu \mathrm{g} / \mathrm{g} \mathrm{DW})$. Proof of concept for the production of astaxanthin was achieved in potato albeit at low levels [270, 271].

Carotenoid levels can also be modulated by creating a metabolic sink, i.e. promoting the formation of carotenoid-sequestering structures. Expression of the cauliflower Or gene [109] in potato confirmed that the protein induces chromoplast differentiation and carotenoid sequestration, resulting in a 6-fold increase in total carotenoids [110]. Furthermore, carotenoids in the generated transgenic lines remained stable for up to 6 months in cold storage leading to further accumulation, i.e. an 18-fold increase at 5 months and a 13-fold increase at 6 months $[110,272]$. Co-expression of $\mathrm{Or}$ with bacterial crtZ and crtW genes improved astaxanthin accumulation in potato tubers up to $77 \mu \mathrm{g} / \mathrm{g}$ DW, 5.5-fold higher than previous attempts [271, 273].

\subsubsection{Sweet potato (Ipomoea batatas)}

Sweet potato is an important tuberous root crop that produces low molecular weight antioxidants such as carotenoids and anthocyanins. Transgenic sweet potato plants constitutively overexpressing Or-Ins (endogenous Or insertion type) in an anthocyanin-rich purple-flesh cultivar exhibited up to 7-fold increased carotenoid levels in their tubers compared to untransformed controls [274]. The levels of zeaxanthin were 12 times higher in the transgenic plants, whereas $\beta$ carotene increased 1.75 times.

\subsection{Oil seeds}

\subsubsection{Canola (Brassica napus)}

Genetic engineering of canola seeds to boost their carotenoid content was a major achievement [275]. Overexpression of bacterial crtB using the seed-specific napin promoter increased total carotenoid levels to more than $1 \mathrm{mg} / \mathrm{g} \mathrm{DW}$, a 50 -fold increase. While $\beta$-carotene accumulation reached $401 \mu \mathrm{g} / \mathrm{g}$ DW, the level of lutein remained unaltered [275]. The combined expression of bacterial $c r t B$ and $c r t /$ further increased total carotenoid levels to $1.4 \mathrm{mg} / \mathrm{g}$ DW with $\beta$-carotene levels reaching $857 \mu \mathrm{g} / \mathrm{g}$ DW [276]. In a more ambitious effort, seven bacterial carotenogenic genes were simultaneously introduced into canola [277]. Two of them encoded enzymes to increase GGPP supply (idi and crtE), three encoded enzymes to convert GGPP into $\beta$ carotene ( $c r t B, c r t l$, and $c r t Y$ ), and two more enabled ketocarotenoid biosynthesis (crtW and $c r t Z$ ). Transgenic seeds expressing all seven transgenes accumulated up to $214 \mu \mathrm{g} / \mathrm{g} \mathrm{FW} \beta$ carotene, a 1070-fold increase over wild type, and they also produced ketocarotenoids rarely 
seen in plants, such as echinenone, canthaxanthin, astaxanthin, and adonixanthin [275]. On a different approach, downregulation of the endogenous $L C Y E$ gene in canola seed increased the levels of $\beta$-carotene, zeaxanthin, violaxanthin and, unexpectedly, lutein, achieving up to 41.7-fold higher concentrations of total carotenoids compared to untransformed seeds [278].

\subsubsection{Soybean (Glycine max)}

Expression of a recombinant sequence encoding pepper (Capsicum annuum) PSY and bacterial Crtl activities linked by a self-processed 2A peptide in Korean soybean (Glycine max L. cv. Kwangan) led to a 62-fold increase in total seed carotenoids (up to $146 \mathrm{mg} / \mathrm{g} \mathrm{DW}$ ), with 112 $\mathrm{mg} / \mathrm{g}$ DW $(77 \%)$ of $\beta$-carotene [279]. Similarly to canola, transgenic soybean plants overexpressing a seed-specific crtB gene accumulated high levels $(845 \mu \mathrm{g} / \mathrm{g} \mathrm{DW})$ of $\beta$-carotene [280]. When the $c r t B$ gene was introduced in combination with $c r t W$ to produce ketocarotenoids, the generated transgenic soybean seeds produced canthaxanthin at levels up to $52 \mu \mathrm{g} / \mathrm{g} \mathrm{DW}$ [281]. Unlike untransformed seeds, which only produce lutein, transgenic seeds also accumulated other carotenoid compounds such as phytoene, lycopene, $\alpha$-carotene, lutein, $\beta$-carotene, and $\beta$ cryptoxanthin. Production of astaxanthin in transgenic soybean seeds has been shown to be possible [281] but accumulation levels were low (7 $\mu \mathrm{g} / \mathrm{g}$ DW in the best line).

\subsection{Cereals}

\subsubsection{Rice (Oryza sativa)}

The endosperm of cereal seeds is a major food staple, but it is deficient in carotenoids. The $\beta$-carotene-rich Golden Rice was engineered by overexpressing plant PSY and bacterial crtl genes $[282,283]$. However, carotenoids were found to degrade when Golden Rice was stored at ambient temperatures [284]. Thus, besides increasing the carotenoid content, another challenge is the proper storage and maintenance of nutritional qualities of the carotenoid-enriched rice. One of the genes responsible for degradation of carotenoids during storage is the lipoxygenaseencoding gene $r$ 9-LOX1 which is also responsible for rice seed quality deterioration [285]. Downregulation of LOX1 activity in the $\beta$-carotene-enriched Golden Rice decreased oxidation of $\beta$ carotene [285]. These results demonstrated that this is a key enzyme for catalyzing oxidation of $\beta$-carotene and that it has a significant role in $\beta$-carotene degradation in rice. Recent efforts have also expressed Or in rice endosperm [112].

Proof of concept for the successful production of astaxanthin in Golden Rice was recently achieved by co-expressing $s C r B K T$, a synthetic $C$. reinhardtii gene encoding $\beta$-carotene ketolase [286]. The resulting grains accumulated predominantly the diketocarotenoids canthaxanthin and adonirubin as well as low levels of astaxanthin. The predominance of canthaxanthin and adonirubin indicated the presence of a hydroxylation bottleneck in the ketocarotenoid pathway. This final rate-limiting step must therefore be overcome to maximize the accumulation of astaxanthin, the end product of the pathway, in rice endosperm [286].

\subsubsection{Maize (Zea mays)}

Transgenic maize endosperm expressing the bacterial $c r t B$ and $c r t$ genes under the control of an enhanced $\gamma$-zein promoter resulted in increase of the total carotenoid content up to 33.6 $\mu \mathrm{g} / \mathrm{g}$ DW [287]. A significant advance was achieved with the development of a combinatorial genetic transformation system designed to dissect and modify the carotenoid biosynthetic pathway in maize, using the white endosperm elite South African inbred line M37W [288]. After randomly introducing into maize five carotenoid-related genes under the control of endospermspecific promoters, analysis of the resulting combinatorial transgenic maize plant population (equivalent to a "mutant series") allowed the identification of rate-limiting steps in the endosperm carotenoid pathway and to recover maize plants with extraordinary levels of $\beta$ carotene and other nutritionally important carotenoids [288]. The total endosperm carotenoid 
content in an elite event (Ph3) co-expressing maize PSY1 and crtl reached $156 \mu \mathrm{g} / \mathrm{g} \mathrm{DW}$, including $57 \mu \mathrm{g} / \mathrm{g}$ DW $\beta$-carotene and $26 \mu \mathrm{g} / \mathrm{g}$ DW lycopene. Simultaneous expression of a LCYB-encoding gene together with PSY1 and $\mathrm{crtl}$ in a different line $(\mathrm{Ph} 4)$ dramatically reduced the levels of lycopene $(11.50 \mu \mathrm{g} / \mathrm{g}$ DW, $7.73 \%)$ but increased the levels of zeaxanthin (34.53 $\mu \mathrm{g} / \mathrm{g} \mathrm{DW}, 23.21 \%$ ) compared to the Ph3 line [288], confirming a rate-limiting role for LCYB in the synthesis of cyclic carotenes. Phytoene, which is absent in M37W endosperm, accumulated in Ph3, Ph4, and in a line (Ph1) expressing only PSY1 but not in the Ph2 line, which does not express PSY1, suggesting that the conversion of phytoene to lycopene is also a limiting step for carotenoid biosynthesis. Line Ph4 was used to introgress the transgenic PSY1-crtl-LCYB mini-pathway into a yellow maize inbred line with a high $\beta: \varepsilon$ carotenoid ratio (A632), resulting in unprecedented amounts of zeaxanthin $(56 \mu \mathrm{g} / \mathrm{g} \mathrm{DW})$ in the hybrids [289]. When the same mini-pathway was introgressed into a different yellow maize inbred with a low $\beta: \varepsilon$ ratio (EP42), resulting hybrids had a very different carotenoid profile, with higher levels of lutein $(23 \mu \mathrm{g} / \mathrm{g} \mathrm{DW})$ compared with the parental lines [289]. Silencing of the endogenous $B C H 1$ and $B C H 2$ genes in the endosperm of a range of maize genetic backgrounds led to up to 4 -fold higher $\beta$-carotene content in all hybrids in which $B C H 2$ was silenced, regardless of whether or not $B C H 1$ was silenced too [290]. These and other results indicated that $\mathrm{BCH} 2$ is the main responsible enzyme for the conversion of $\beta$-carotene to zeaxanthin in maize endosperm [290].

The overexpression of Arabidopsis Or in the white-endosperm M37W line raised the total carotenoid content of the endosperm to $25 \mu \mathrm{g} / \mathrm{g}$ DW, a 32-fold increase, without changing the relative proportion of lutein and zeaxanthin [113]. Crossing the best-performing Oroverexpressing line with four other transgenic parental lines accumulating different levels of carotenoids and/or ketocarotenoids yielded hybrids with the same qualitative carotenoid profiles as their parents. The total carotenoid levels in the hybrids increased when the carotenoid pool in the parent was restricted but there was no significant change when carotenoids were already abundant (above $70 \mu \mathrm{g} / \mathrm{g} \mathrm{DW}$ ) in the parent [113].

In early proof of concept experiments, limited astaxanthin accumulation in transgenic maize plants was attributed to a bottleneck in the conversion of adonixanthin (4-ketozeaxanthin) to astaxanthin [288]. More recently, transformation of $\mathrm{M} 37 \mathrm{~W}$ maize with constructs to overexpress PSY1, crtZ and sCrBKT and knock-down LCYE was successful to direct the pathway towards the production of astaxanthinin [291]. This astaxanthin-accumulating transgenic line was further crossed with a high-oil maize line (NSL76) in order to increase the storage capacity for lipophilic astaxanthin. The resulting hybrid line accumulated $60 \%$ of total seed carotenoids as astaxanthin, reaching levels of $16 \mu \mathrm{g} / \mathrm{g}$ DW [291].

\subsubsection{Other cereals}

Sorghum (Sorghum bicolor) is deficient in $\beta$-carotene. The co-expression of genes encoding plant DXS and PSY enzymes with crt/ in sorghum endosperm resulted in up to 10 -fold increase of $\beta$-carotene, but with a half-life of less than 4 weeks [292]. Further co-expression of barley homogentisate geranylgeranyltransferase, however, significantly improved $\beta$-carotene accumulation and stability, extending its half-life from 4 to 10 weeks in sorghum grains [292].

In order to enrich the carotenoid content in wheat (Triticum aestivum) grains, crt/ was coexpressed with PSY1 or crtB in wheat endosperm, resulting in a total carotenoid content of 5 $\mu \mathrm{g} / \mathrm{g}$ DW, a ca. 10-fold enrichment [293, 294]. Overexpression of $c r t B$ together with endospermspecific silencing of an endogenous $B C H$ gene (TaHYD) was also successful to increase $\beta$-carotene content to $2.45 \mu \mathrm{g} / \mathrm{g}$ DW in wheat endosperm [295].

\section{Nutrition and health}

A relevant property of carotenoids for human nutrition is their use as vitamin A precursors. Provitamin $A$ activity, however, is restricted to carotenoids with at least one unsubstituted $\beta$ ring, such as $\beta$-carotene (the most potent dietary precursor of vitamin $A$ as it shows two unsubstituted 
$\beta$ rings), $\alpha$-carotene, $\beta$-cryptoxanthin, and other carotenoids scarcely found in our diets (Figure 4). Additionally, epidemiologic studies associate a carotenoid-rich diet (usually resulting from high consumption of fruits and vegetables) with health benefits. These beneficial properties of dietary carotenoids have stimulated an interest on carotenoid food sources, bioavailability and mode of action, as well as on their use in functional food and nutraceutical products. It is noteworthy, however, that only a fraction of the carotenoids ingested in our diet have been studied in depth, which means that there is much room to gain insight into the properties, actions and practical applications of dietary carotenoids.

\subsection{Health benefits}

Interest in carotenoids from a nutritional and physiological point of view has been focused mainly on the provitamin A activity of carotenoids containing $\beta$ rings, as vitamin $A$ deficiency is one of the major micronutrient deficiencies worldwide. In mammals, provitamin A carotenoids are converted into retinol and other related retinoids such as 11-cis-retinal and retinoic acid, which play important roles in the visual cycle and in gene regulation, respectively [296]. Additionally, carotenoids display other biological activities that may confer beneficial effects but whose relevance for human health is, in general, still uncertain [297-300]. Healthrelated benefits associated with a high carotenoid intake include enhanced immune system functions and lower risk of developing degenerative chronic diseases, such as age-related macular degeneration, type 2 diabetes, obesity, certain types of cancer (breast, cervical, ovarian, colorectal), and cardiovascular diseases, among others [301-308]. Carotenoids are also attracting interest as promoters of cognitive functions [309-311] as well as skin photoprotectants and providers of cosmetic benefits, particularly in the context of the rapidly expanding nutricosmetics area [312]. Because carotenoids may undergo significant degradation and/or metabolism in the human body, it is far from certain whether the native compounds or rather their metabolites are responsible for the attributed health effects.

Epidemiological data linking high carotenoid intake with improved health have inspired several intervention trials in humans which have emphasized that, while typical dietary intakes of carotenoids via fruits and vegetables appear to exert rather beneficial effects on health, supplemental intake with doses above $20 \mathrm{mg} / \mathrm{d}$ of purified or synthetic $\beta$-carotene may result in adverse effects, including increased cancer risk and total mortality [313]. The hypothesis that $\beta$ carotene may decrease cancer risk was tested in the Alpha-Tocopherol Beta-Carotene (ATBC) trial, which included 29,000 participants who received a combined daily supplement of $20 \mathrm{mg} / \mathrm{d}$ of $\beta$-carotene together with other micronutrients for 5-8 years [314]. By the same time, this hypothesis was also tested in the Carotene and Retinol Efficacy (CARET) trial [315], which included $\sim 18,000$ participants who received a combined daily supplement of $30 \mathrm{mg} / \mathrm{d}$ of $\beta$ carotene for 4 years on average. The ATBC and the CARET studies, where smokers and asbestos exposed subjects were supplemented with purified or synthetic $\beta$-carotene, surprisingly found increased, rather than decreased, lung cancer risk [315, 316]. The CARET study also reported an increased mortality due to coronary heart disease after $\beta$-carotene supplementation [315]. In both studies, $\beta$-carotene was given as supplements, resulting in much higher (up to 10 times higher on average) blood concentrations compared to the USA 95\% percentile concentration [317]. The outcome of ATBC and CARET studies contrasts with a study with 22,000 non-smoking male physicians where the subjects were supplemented with $50 \mathrm{mg} \beta$-carotene every alternating day [318]. In this study, $\beta$-carotene supplementation did not result in any observed detrimental effects despite increasing $\beta$-carotene circulating levels [318]. Similarly, the Chinese Linxian intervention trial with 30,000 men and women, primarily non-smokers, reported preventive effects for stomach cancer and total mortality of a combined vitamin and mineral supplement including $15 \mathrm{mg} / \mathrm{d} \beta$-carotene [319]. All these studies added to the controversy regarding carotenoid health benefits and safety, emphasizing that dosing, form of administration (food matrix or supplement), treatment duration, combination with other nutrients, and subject individual factors (e.g., healthy vs. sick, nutritional status) are likely important modifiers [296, 
320]. In any case, the main take-home message is that dietary supplements of synthetic or purified carotenoids are not as effective in promoting health benefits as the intake of carotenoids in food products, where they interact with other cellular components.

The interaction of carotenoids and retinoids with the control of lipid and energy metabolism in mammals is receiving an increasing attention due to its potential implications for chronic disorders including obesity, diabetes, nonalcoholic fatty liver disease and atherosclerosis $[304,321,322]$. A recent prospective study in 1042 nondiabetic Chinese adults found an inverse association between serum RA levels at baseline and the incidence of metabolic syndrome during a 4-year follow-up [323]. Evidence from animals and cell studies has accumulated that retinoids such as RA impact on developmental and biochemical processes influencing mammalian adiposity. Modulated processes include adipocyte differentiation, lipogenesis, adaptive thermogenesis, lipolysis, and fatty acid oxidation in tissues such as liver, white and brown adipose tissues, and skeletal muscle [321]. Retinoids effects on these processes converge in an overall anti-adiposity action. In line with these findings, expression of uncoupling protein 1 (whose activity constitutes the molecular basis of adaptive thermogenesis) was promoted by carotenoids in cultured brown adipocytes, with an effectiveness that was related to their potency as retinoid precursors [324]. Furthermore, dietary $\beta$-carotene supplementation reduces adiposity in mice in a BCO1-dependent manner [325]. Other carotenoids with anti-adiposity activity in preclinical models include $\beta$-cryptoxanthin, fucoxanthin and astaxanthin [304]. Importantly, antiadiposity action has been documented in pilot human intervention trials testing supplements enriched in these carotenoids [304].

Among the non-provitamin A carotenoids, lutein and zeaxanthin deserve special attention as they are preferentially accumulated in the retina, together with meso-zeaxanthin, and they improve eye health. These three xanthophylls are collectively known as macular pigment, functioning as blue-light filters and as antioxidants that protect the retina from oxidative damage induced by light [326-330]. There is evidence of a protective role of lutein and zeaxanthin against age-related macular degeneration, a major cause of blindness in the elderly [326, 328, 331, 332]. Moreover, they also accumulate in brain tissue and there is increasing evidence that they provide cognitive benefits [309-311]

\subsection{Dietary sources}

\subsubsection{Carotenoids in the diet}

Carotenoids present in food and feed products are usually C40, although some examples with shorter carbon number can also be found (including apocarotenoids and other CCPs). Most of the dietary carotenoids are obtained from a limited number of fruits and vegetables, but the contribution of animal-derived food should not be overlooked [308]. In fact, egg yolk, dairy products (milk, butter, etc.) and seafood contain high levels of carotenoids such as lutein, $\beta$ carotene, zeaxanthin, astaxanthin, or canthaxanthin (see Section 2.2.5 above). Carotenoids can also be taken in the diet as food supplements or additives. In any case, our normal diet only covers a fraction of all carotenoid diversity. It has been estimated that our daily food intake only contains about 50 carotenoids that can be absorbed and used in our bodies [308]. In the human blood plasma, however, this number is reduced to around 20, from which the major ones are $\beta$ carotene, $\alpha$-carotene, $\beta$-cryptoxanthin, lycopene, lutein and zeaxanthin [333]. The largely ignored colorless carotenoids phytoene and phytofluene are also major circulating carotenoids [31]. Furthermore, a significant number of different geometric isomers for all these carotenoids have been reported [334].

The presence and distribution of the most common carotenoid pigments found in generally consumed foods, mostly fruit and vegetables, are shown in Table 2. Most fruits and vegetables contain $\beta$-carotene, including carrots, red bell peppers, oranges, sweet potatoes, broccoli, and all green vegetables $[308,335]$. The best sources of $\alpha$-carotene are carrots, palm oil fruit and pumpkins, where it is always accompanied by $\beta$-carotene [308]. $\beta$-cryptoxanthin is widely found 
at low concentration in fruits but it is a major carotenoid in ripe red and orange peppers, papayas, oranges, mandarins, mangos and persimmons [336-339]. Lutein is the major pigment in green vegetables such as spinach, Brussels sprouts, broccoli, and pea, whereas zeaxanthin is found in high concentrations in red pepper, goji berries, corn and egg-yolk [336, 340-342]. The main sources of lycopene are tomato fruits and derived products (pasta and sauces), together with watermelon and pink grapefruit $[308,343]$. The benefits of carotenoids for human health have stimulated the search for new natural sources, including colorful exotic (tropical) and wild fruits and vegetables which may additionally supply other phytochemicals with nutritional properties and health benefits [344-348]. In parallel, selection and breeding of carotenoid-rich cultivars of traditional staple food crops such as potato or wheat will serve to enrich our diet in carotenoids and possibly other health-promoting phytonutrients [349-351].

The green parts of plants (including leaves, stems, and green seeds and fruits) contain typical chloroplast-associated carotenoids. Besides lutein, $\beta$-carotene, violaxanthin, and neoxanthin, smaller amounts of $\alpha$-carotene, $\gamma$-carotene, $\beta$-cryptoxanthin, zeaxanthin, antheraxanthin and lutein 5,6-epoxide can also accumulate in these tissues. In some special cases, such as lettuce, an unusual dihydroxylated xanthophyll bearing two $\varepsilon$-rings (lactucaxanthin) is found. In contrast, non-green tissues of ripe fruits, seeds, and tubers have a highly diversified carotenoid profile depending on the species, the developmental stage, or the environmental conditions. Whereas xanthophylls are found free (non-esterified) in green leaves, they are esterified with fatty acids in many ripe fruits and some leaves and tubers [352-354]. In fact, xanthophylls esterified with fatty acids are the main carotenoid form in most fruits [352]. However, the presence of carotenoid esters has been often overlooked by many authors, mainly due to the use of saponification as a routine step in carotenoid analysis [355]. Consequently, information regarding carotenoid ester contents in fruits, vegetables and other plant materials (flowers, seeds and tubers) is scarce [24]. The acylation of xanthophylls with fatty acids seems to be an important part of the process used by plants to over-accumulate these pigments in carotenogenic fruits and senescing vegetables [354], enhancing and stabilizing their external color. This represents a vital role in attracting animals (including humans) to act as pollinators and seed dispersion vehicles [97]. The esterification of xanthophylls also appears to improve their stability against thermooxidative processes associated with food processing and to increase their bioavailability through a better solubilisation and extraction (bioaccessibility) during digestion in presence of dietary fat [356, 357].

Besides genetic determination, the actual composition of carotenoids in plant products also depends on stage of maturity, variety or cultivar, harvesting season, production practices, post-harvest handling, processing and storage. Furthermore, differences among cultural and dietary habits, together with cooking, processing and preservation methods may profoundly affect the carotenoid content of foods. Heat treatments typically increase the chemical extractability of carotenoids (e.g. by softening membranes or cell walls), resulting in apparently higher levels of carotenoids in processed food compared to raw products [342, 358]. Another source of error is that the changes in the weight of the processed or cooked food are not taken into account, and so the reported retentions of carotenoids are not realistic. In this sense, several formulae for their calculation are recommended [358]. Processing, cooking and/or storage can also cause geometrical isomerisations $[359,360]$ and 5,6-epoxide to 5,8-furanoid rearrangements $[17,361]$ that are accompanied by changes in the light absorption spectra, hence explaining the noticeable food color changes that are often associated to these processes [358].

\subsubsection{Classification of food sources}

As indicated above, the carotenoid content in foods depends on a great diversity of factors of different nature. Hence, the levels of carotenoids in a particular food can vary to a great extent. As a general guide, the carotenoid content of different food matrices has been compiled in several studies and databases such as the USDA Food Composition Database (https://ndb.nal.usda.gov/ndb) and others [335, 362-365]. The qualitative and quantitative 
information gathered by such food composition databases provides a good hint on the type and amount of food supplying the adequate amount of a specific carotenoid. According to the criteria proposed by Britton and Khachik, the levels of a specific carotenoid in food products can be classified as low $(0-0.1 \mathrm{mg} / 100 \mathrm{~g})$, moderate $(0.1-0.5 \mathrm{mg} / 100 \mathrm{~g})$, high $(0.5-2 \mathrm{mg} / 100 \mathrm{~g})$ or very high (> $2 \mathrm{mg} / 100 \mathrm{~g}$ ) [308]. Food sources with high or very high carotenoid levels include green vegetables, apricot, carrot, mango, palm oil, buriti and sweetpotato for $\beta$-carotene, persimmon, pitanga, papaya, pumpkin and tangerines for $\beta$-cryptoxanthin, green vegetables, pumpkin, sastra, and egg yolk for lutein, Chinese wolfberry, sastra, corozo, sapote, quince, orange and red peppers for zeaxanthin, or tomato, watermelon, red grapefruit and papaya for lycopene $[308,348]$.

Five distinctive carotenoid patterns have been proposed in relation to the color of the plant tissue [308, 366]: (1) large amounts of the acyclic carotene lycopene, as in tomatoes (red color); (2) large amounts of $\beta$-carotene and/or its hydroxyl derivatives $\beta$-cryptoxanthin and zeaxanthin (orange color); (3) similar to pattern 2 but presenting also $\alpha$-carotene and/or its hydroxyl derivatives, especially lutein (yellow-orange color); (4) large amounts of carotenoid epoxides (yellow color); and (5) carotenoids that appear to be unique to or characteristic of that species, e.g. capsanthin and capsorubin in red peppers (yellow, orange, or red color). Again, the carotenoid composition and, subsequently, the color vary in different cultivars. Some popular examples besides peppers are tomatoes (those containing lycopene as the major pigment are red but there are cultivars with orange fruits accumulating $\beta$-carotene or yellow fruits containing lutein) and carrots (orange when accumulating $\alpha$-carotene and $\beta$-carotene but red or yellow when accumulating lycopene or lutein, respectively) [308]. Colorless phytoene and phytofluene have been largely ignored in the context of food science, but there is a growing interest for them in the fields of nutrition, health and even nutricosmetics [31]. High contents of these colorless carotenoids are found in tomato, carrot, apricot, red grapefruit, and red peppers [31, 342, 363].

\subsubsection{Dietary intake recommendations}

It is important to note that high carotenoid contents not always classifies a given food as a good contibutor to dietary carotenoid intake. Besides bioavailability issues (see Section 4.3 below), the intake frequency is key to evaluate the main dietary contributors to the intake of the carotenoid [362]. Thus a food can have a very high content of a given carotenoid but can be scarcely consumed in the diet (e.g. exotic fruits in Western diets), whilst others can have a modest content but be taken very often (e.g. corn or bananas in certain countries). The total carotenoid intake in European countries (median values) ranges from $\sim 9.5$ to $16 \mathrm{mg} / \mathrm{d}$ ( $\sim 3$ to 6 $\mathrm{mg} / \mathrm{d}$ for $\beta$-carotene), being the major contributing food sources vegetables, followed by fruits and soups/bouillon $[362,367]$. The carotenoid dietary intake from a selection of studies is shown in Table 3. The concentration of carotenoids in serum is considered to reflect short-term dietary intake and it is widely accepted as a good biomarker of fruit and vegetable intake [368, 369].

No specific recommendations regarding the intake of carotenoids have been issued to date. Recommendation for provitamin A carotenoid intake for subjects not consuming other sources of this vitamin (e.g. animal sources) to fulfill its recommended dietary allowance (RDA) can be calculated as $\sim 10.8 \mathrm{mg} / \mathrm{d}$ of $\beta$-carotene or twice as much $(\sim 21.6 \mathrm{mg} / \mathrm{d})$ of other provitamin A carotenoids with only one unsubstituted $\beta$ ring (Figure 4). This is expected to provide the RDA for healthy adult males and women: 900 and $700 \mu \mathrm{g}$ retinol (vitamin A) activity equivalents per day, respectively. Although no RDA exists for lutein, there is increasing consensus that this carotenoid meets the criteria for non-essential bioactives regarding the promotion of optimal health and/or prevention of chronic diseases to be considered for intake recommendations [370]. Intakes of up to $10 \mathrm{mg} / \mathrm{d}$ of lutein and zeaxanthin have been advocated for some population segments [371]. In any case, recommendations for dietary carotenoid intake are impeded (1) by the fact that carotenoid bioavailability depends on a number of dietary as well as host-related factors that lead to inter-individual variability in absorption and metabolism [369], 
and (2) by the scarcity of available information on the metabolic conversions of carotenoids in mammals.

\subsection{Bioavailability}

Bioavailability is defined as the fraction of a nutrient (e.g. carotenoid) that is absorbed and available for utilization in normal physiological functions or for storage $[372,373]$. Carotenoid bioavailability is influenced by a combination of dietary and physiological factors and varies depending on the type of carotenoid, the food matrix in which the carotenoid is incorporated, and host-related factors [297, 372, 374]. Bioavailability encompasses other terms such as bioaccessibility, bioconversion, and bioefficacy. Bioaccessibility is the amount of the carotenoid released from the food matrix during digestion and made available for absorption. Bioaccessibility takes into account factors such as carotenoid species, food matrix, chemical state, amount ingested, and absorption modifiers, which can be studied by means of in vitro methodologies (including in vitro digestion, cellular and in silico models). These technologies are simple, low cost and reproducible, and they help to predict (to some extent) the bioavailability of food components [297, 374-376]. However, the information provided by bioaccessibility studies is incomplete as it does not include data on host-related factors, which hence need to be studied in vivo.

Host related factors condition bioconversion and bioefficacy. For provitamin A carotenoids, bioconversion is traditionally defined as the proportion of absorbed carotenoid converted to retinol. Thus, a bioconversion rate of $100 \%$ means that all of the absorbed $\beta$-carotene is converted to retinal and then reduced to retinol (Figure 9). Bioefficacy is the efficiency with which ingested dietary carotenoids are absorbed and bioconverted to active retinol. A $100 \%$ bioefficacy means that all $\beta$-carotene taken in the diet has been absorbed and converted to retinol [377]. Host factors related to intra- and inter-individual differences in carotenoid absorption, distribution, metabolism and excretion have been thoroughly reviewed $[297,375]$. The observed variability can be attributed to age, weight, disease state, physical activity, alcohol use, smoking habits, drug intake, or genetic aspects, but also to microbiota, factors influencing absorption such as parasites, or concentration of enzymes and other components involved in digestion [369].

\subsubsection{Estimating bioaccessibility in vitro}

In vitro methods to determine bioaccessibility attempt to mimic physiological conditions in vivo, simulating the digestion processes. They typically include the oral, gastric and small intestine phases, and, occasionally, large bowel fermentation. In vitro digestion models coupled with Caco-2 cells are also used in this assessment [374, 378, 379]. Models of simulated digestion process that mimic the peristaltic movements and body's temperature are also available [380]. However, the majority of the models and methods reported in the literature are static. While they take into account the presence of digestive enzymes, $\mathrm{pH}$, digestion time and salt concentrations, among other factors, they vary widely in the use of those parameters, making it quite difficult to compare results [376, 381]. A standardized digestion method, with conditions and composition of digestive fluids supported by a broad consensus in terms of physiological relevance, was recently published [381]. This consensus method was adapted to assess the bioaccessibility of provitamin A carotenoids in fruits, showing a similar or higher $\beta$-cryptoxanthin bioaccessibility than that of $\beta$-carotene in the fruits analyzed [382].

\subsubsection{Estimating bioavailability in vivo}

Bioavailability usually refers to in vivo studies in animals, the gold standard being the studies in humans. Among the latter, those most frequently used are short-term interventions (that is, single-dose, pharmacokinetic studies) and long-term interventions (involving repeated doses). In human intervention studies, different aspects regarding the subjects, methods, 
biomarkers, and statistical analyses are crucial and need to be properly documented for a reliable and precise information on host-related factors and to allow the comparison among studies. The research question has to be clearly identified and the subjects well characterized (e.g. inclusion/exclusion criteria, representative sample, nutritional status). The methods should include clearly defined outcomes and adequate, valid and reliable markers. Markers for carotenoid bioavailability can be broadly divided into direct or biochemical markers (that usually refer to biochemical indicators of intake and/or status) and, indirect or physiological indicators (that should reflect cumulative exposure, biological effect or modification of risk factor) [383]. Finally, the study design and type of outcome indicators should be subjected to appropriate statistical analysis. Information should be included on all the variables known to have a possible influence on carotenoid metabolism (e.g. as inclusion criteria or as variables that could be used in the statistical analysis of the results).

Another way to determine bioavailability is by using data from food intake and serum carotenoid levels. The blood carotenoid concentration in each individual (an indicator of the absorption capacity and corporal retention of the carotenoid) is divided by its corresponding intake, allowing to assess the apparent bioavailability. In the case of provitamin A carotenoids, current assumptions about retinol activity equivalent (RAE) provided for different dietary carotenoids are mainly based on data on $\beta$-carotene bioavailability and on the theoretical $50 \%$ yield of carotenoids with a single $\beta$ ring such as $\alpha$-carotene and $\beta$-cryptoxanthin [384]. But there is a wide variation in bioconversion factors (on a weight basis) for dietary $\beta$-carotene to retinol assessed in human studies, which is in the range from 3.6:1 to $28: 1$, with Golden Rice and leafy vegetables, respectively [320]. On the comparison of results from these studies, special attention has to be paid to factors such as the food matrix (e.g. fruits, vegetables, complete meal, isotopelabeled food), amount ingested (higher intakes lead to lower conversion rates), characteristic of the subjects (e.g. with normal or marginal vitamin A status), and methods to assess the conversion (e.g. area under curve responses, changes of serum retinol concentration, changes of total body stores of vitamin A). The bioconversion of $\beta$-carotene into retinol is highly influenced by the food matrix, and the rate seems to be higher in fruits than in green vegetables. Interestingly, the apparent bioavailability of $\beta$-cryptoxanthin from typical western diets (mainly present in fruits as ester forms) appears to be greater than that of $\alpha$-carotene or even $\beta$-carotene $[339,385]$.

\subsection{Absorption}

The absorption and metabolism of carotenoids (particularly $\beta$-carotene) in mammals has been addressed in detail in several reviews [386-391] and it is briefly summarized here. Early after ingestion of food, mastication, gastric action and digestive enzymes release carotenoids from the food matrix. Since carotenoids are nonpolar lipid compounds that cannot dissolve in aqueous solutions, they are incorporated in the stomach into mixed micelles consisting of lipids and bile components to be delivered to the apical surface of the enterocytes, the absorptive epithelial cells of the small intestine. The amount of carotenoid incorporated into micelles depends on the polarity of the carotenoid and on micellar lipid composition and saturation. Recently, it has been demonstrated that the geometry of the molecules can be even more important than polarity. Thus, the colorless carotenes phytoene and phytofluene, whose shapes are less rigid for having much fewer conjugated double bonds and being in a cis configuration, are much more efficiently released from different juices than major xanthophylls occurring in them [392].

Non-provitamin A carotenoids (lycopene, lutein, zeaxanthin) can be absorbed intact or undergo oxidative cleavage before absorption from the intestinal lumen. Lutein and zeaxanthin esters (common in fruits and vegetables) are also hydrolyzed by pancreatic lipases in the lumen of the small intestine before their uptake [393]. Then, carotenoids are absorbed in free (unesterified) form by the enterocyte (mainly in the duodenum) via passive diffusion or through facilitated transport via scavenger receptor class B member 1 (SR-BI) and possibly other lipid 
transporters, such as CD36. Once taken by the enterocytes, carotenoids are incorporated into large lipoprotein particles (nascent chylomicrons) for secretion [394], or can undergo enzymatic cleavage (Figure 9). Retinol derived from dietary $\beta$-carotene undergoes esterification to longchain fatty acids, and is also packaged as retinyl esters in nascent chylomicrons [387]. The efficiency of intestinal $\beta$-carotene cleavage varies greatly across species. Adult rats and mice are considered efficient cleavers that convert most absorbed $\beta$-carotene into retinol. However, even following mild acute or chronic oral $\beta$-carotene supplementation, some $\beta$-carotene is absorbed intact in adult rodents, as revealed by accumulation in tissues $[325,395]$. In young (suckling) rats, absorption of intact $\beta$-carotene occurs even at lower dietary doses, probably due to their immature intestine [396]. In humans and other species such as horses and ferrets about $17 \%$ $45 \%$ of the ingested $\beta$-carotene escapes intestinal cleavage, is incorporated into chylomicrons and is found associated with circulating lipoproteins. Interestingly, intestinal $\beta$-carotene absorption and cleavage are dependent on the animal's vitamin A status through a feedback mechanism [397].

Chylomicrons with their packaged carotenoids and retinyl esters are released by exocytosis to the lymphatic system for delivery to the bloodstream and eventually directed to the liver. Before reaching this organ, partial degradation by the activity of the endotheliumassociated lipoprotein lipase (LPL) causes liberation of part of the lipid content of the particles, including carotenoids. Most carotenoids reach the liver in chylomicron remnants. There, they are stored or secreted back into the bloodstream associated with very low density lipoproteins (VLDL). VLDL are converted into intermediate-density lipoproteins (IDL) and low-density lipoproteins (LDL) after being processed by LPL in the extrahepatic capillaries and exchange components with other lipoproteins. In the fasting state, most plasma carotenoids are associated with LDL and, to a lower extent, with high density lipoproteins (HDL). HDL particles are produced in the liver and intestine and are secreted into the blood and lymph, respectively. Carotenoids associated with HDL may therefore derive from the liver, directly from the gut, or from exchange between lipoproteins [398]. Apolar carotenoids such as lycopene and $\beta$-carotene are found mostly associated with LDL, whereas lutein and zeaxanthin are more evenly distributed between $\mathrm{LDL}$ and HDL fractions. A reason for this distribution might be that lycopene and $\beta$-carotene are mainly found in the core of the lipoprotein, whereas the more polar xanthophylls are mainly located on the surface. Therefore, transfer between lipoproteins might be easier for lutein and zeaxanthin, resulting in a more equal balance between LDL and HDL. As illustrated in Figure 10, carotenoids and retinyl esters contained in circulating lipoproteins are taken up by tissues through the action of lipoprotein-specific receptors, such as SR-BI (the receptor for HDL), LDL receptor (LDLr), LDLr-related protein-1, or LPL [389]. Membrane transporters may also be implicated, such as CD36, which mediates the uptake of lycopene by adipocytes [399]. Liver, followed by adipose tissue, kidney, skin and lung are important sites of accumulation of $\beta$ carotene and other carotenoids [389]. In addition, there is a very specific deposition of lutein and zeaxanthin within the eye, suggesting a biological process governing their capture, deposition, and stabilization in the macula. This regulation or control is believed to be elicited by binding proteins [400].

Liver is also a major site for vitamin A storage, in the form of retinyl esters in stellate cells. Mobilization of vitamin A from hepatic stores depends on the 21-kDa retinol-binding protein (RBP, also known as RBP4), which is produced in hepatocytes and secreted into the circulation in a retinol-dependent manner. Circulating retinol-RBP complexes may be taken up by extrahepatic tissues or recycled back to liver multiple times. Uptake by cells relies on specific receptors of the retinol-RBP complexes (Figure 10). Extrahepatic organs such as the eye use the plasma membrane protein STRA6 (stimulated retinoic acid 6) as a high affinity RBP receptor for retinol uptake $[401,402]$. STRA6 is also involved in mediating cellular responses to retinol-RBP complexes related to the activation of the JAK-STAT pathway [402, 403]. A second receptor de retinol-RBP is RBP receptor-2 (RBPR2), which is expressed primarily in liver and intestine and induced in adipose tissue of obese mice [404]. RBPR2 is structurally related to STRA6 and highly 
conserved in vertebrates, including humans. Inside the cells, carotenoids and derived products are metabolized as described in the next Section.

\subsection{Metabolism in mammals}

Mammals possess at least two $\beta$-carotene cleavage enzymes: $\beta$-carotene-15,15'oxygenase (BCO1) and $\beta$-carotene- $9^{\prime}, 10^{\prime}$-oxygenase $(\mathrm{BCO} 2)$, which are expressed in the enterocytes and other tissues [390]. Studies in knockout mouse models indicate that BCO1 is the key enzyme for the production of retinoids with vitamin A activity from dietary $\beta$-carotene $[405$, 406]. BCO1 cleaves $\beta$-carotene centrally into two molecules of retinal (also named retinaldehyde), which can be further oxidized irreversibly to retinoic acid (RA), or reduced reversibly to retinol (Figure 9). Lecithin:retinol acyltransferase (LRAT) and possibly other acyltransferases can esterify retinol to fatty acids for storage of retinyl esters (RE) in lipid droplets (Figure 10). Retinol can also be reversibly metabolized to retinal via alcohol dehydrogenases (ADHs) and retinol dehydrogenases (RDHs) (Figures 9 and 10). BCO2 cleaves $\beta$-carotene asymmetrically to generate diverse $\beta$-apocarotenals and $\beta$-apocarotenones (Figure 9 ). BCO1 can convert some BCO2 cleavage products, in particular $\beta$-apo-10'-carotenal, into retinal [405]. BCO2 products may also have biological activity on their own [387]. The two $\beta$-carotene cleavage enzymes further differ in their substrate specificity and cellular location. While BCO1 is specific for provitamin A carotenoids with at least one non-substituted $\beta$ ring (such as $\beta$-carotene, $\alpha$ carotene and $\beta$-cryptoxanthin), $\mathrm{BCO} 2$ has much broader substrate specificity and metabolizes, for instance, the acyclic carotene lycopene and oxygenated carotenoids (i.e. xanthophylls, such as lutein and zeaxanthin) besides cyclic carotenes [390]. BCO1 is a cytosolic protein, whereas BCO2 is localized to mitochondria (Figure 10), where it contributes to avoid harmful consequences of local carotenoid accumulation on mitochondrial function [407-409].

Intracellular metabolism of carotenoids and derived products takes place with these metabolites bound to specific binding proteins (Figure 10), which may contribute to direct them to specific metabolizing enzymes and targets. Besides carotenoid binding proteins (CBPs), they include cellular retinol-binding proteins (CRBPs) as well as cellular RA binding proteins (CRABPs) and fatty acid binding protein 5 (FABP5), which deliver RA to specific nuclear receptors within the nucleus or to cytochrome P450 (CYP) enzymes for degradation [410]. Some of the components of the RA biosynthetic and catabolic machinery are regulated by RA itself, via feedback regulation loops. Although intracellular carotenoid metabolism in mammalian tissues is still poorly understood, it should be emphasized that $\mathrm{BCO} 1$ and $\mathrm{BCO} 2$ are expressed in many tissues, besides the intestinal mucosa. This widespread expression, together with the wide distribution of carotenoids within the body, has suggested that local tissue-specific conversion of carotenoids may contribute to the in situ generation of retinoids and other CCPs that may impact tissue metabolism and signaling pathways [390].

\subsection{Mode of action}

Besides specific cases such as that of macular pigments, which filter (or absorb) blue light and thus physically protect the underlying photoreceptor cell layer from light damage [330], common biological mechanisms of action may underlie the proposed health benefits of carotenoids. These mechanisms include (A) direct antioxidant and prooxidant activity, (B) activation of the nuclear factor erythroid 2-related factor 2 (Nrf2) signaling pathway, (C) suppression of the nuclear factor $\kappa B(N F-\kappa B)$ signaling pathway, and (D) interaction with other transcription factors, notably members of the nuclear receptor (NR) superfamily of ligandmodulated transcription factors, among others [307, 411-415].

\subsubsection{Antioxidants and prooxidants}

Oxidative stress due to the accumulation of excess ROS has been related to malignant transformation of cells. Hence, antioxidants are usually viewed as preventive agents against 
cancer development [416]. Carotenoids have direct antioxidant activity, i.e. radical quenching properties [411, 413,414]. But while carotenoids are very efficient quenchers of singlet oxygen in simple solutions, their interactions in vivo are more complex as carotenoid aggregation or spanning of cell membranes can reduce their quenching efficiency. Carotenoids can also quench other free radicals to form carotene radicals with different properties from the original carotenes [417]. In the eye, lutein and zeaxanthin act as light filters but also as antioxidants to protect against the formation of ROS [327, 328].

Carotenoids can also be prooxidant in cells under certain conditions, e.g. when highly reactive carotenoid breakdown products (including aldehydes such as retinal and epoxides) formed in the course of antioxidant action accumulate, or when carotenoid detoxifying mechanisms are compromised [418-420]. Studies in BCO2-deficient mice indicate that carotenoids in excess, if not properly detoxified through BCO2 activity, accumulate in mitochondria and induce oxidative stress leading to mitochondria dysfunction and subsequent activation of the intrinsic apoptotic pathway $[407,409]$. In addition, carotenoids may have antioxidant or prooxidant properties depending on the type of radical involved in damaging effects, as seen for $\beta$-carotene in lung epithelial cells with chemical induced genotoxicity [421]. Importantly, prooxidant activity can be beneficial under certain circumstances. Increased ROS production can protect from cancer development by increasing the oxidative stress/damage response leading to apoptosis of the damaged cells [416]. In fact, the increase in ROS production induced by carotenoids such as $\beta$-carotene and lycopene in human colon adenocarcinoma and leukemia cells is highly coincident with their ability to induce apoptosis and cell cycle arrest [418, 422]. It might be added that reduction of ROS by antioxidants can lead to the survival of preinitiated tumor cells [423]. Likewise, the increased administration of antioxidants during cancer progression and treatment may increase the cancer cell survival rate by enhanced resistance against oxidative stress and decreased apoptosis [424]. Thus, both antioxidant and prooxidant activities of carotenoids might be of interest in cancer, and the effects of carotenoid administration could differ depending on the stage of malignant transformation.

Antioxidant as well as antiinflammatory activities of carotenoids or their derivatives might also be of relevance in regard to obesity, as obesity entails the development of oxidative stress and inflammation in adipose tissue [425, 426]. Many components of the classical inflammatory response, such as the induction of proinflammatory cytokines and macrophageattractant signals (chemokines), are activated in the obese adipose tissue. Adipose tissue oxidative stress and inflammation are thought to play a major role in the onset of insulin resistance [427, 428]. These cascades could be interrupted by carotenoids and carotenoid derivatives. In fact, there is evidence that RA and carotenoids such as lycopene and fucoxanthin and their metabolites inhibit proinflammatory cytokine and chemokine expression in cultured adipocytes and adipose tissue under inflammatory conditions [304, 429, 430]. Likewise, mechanisms of action of lutein and zeaxanthin in relation to cognitive function, though not fully elucidated, may relate to their antioxidant and anti-inflammatory capacities among others [311, 414].

\subsubsection{Activators of the Nrf2 pathway}

Besides ROS scavenging, there is increasing awareness that carotenoids, or their polar degradation products or metabolites, may indirectly elicit antioxidant activity in cells through the activation of the Nrf2-dependent antioxidant response (Figure 10). In normal conditions, Nrf2 is associated with Kelch-like ECH-associated protein 1 (Keap 1), a cytoplasmic repressor of Nrf2 that serves as a molecular sensor of intracellular redox status. Redox stress disrupts the Keap1-Nrf2 interaction, allowing Nrf2 to translocate to the nucleus to ensure proper and timely expression of genes that are involved in cellular antioxidant defense or detoxification. There is evidence that lycopene and other carotenoids may exert anticancer effects by up-regulating cellular detoxification enzymes [412, 431-433]. Three lycopenoids (apo-10'-lycopenoic acid, apo-10'- 
lycopenol and apo-10'-lycopenal) were shown to activate the Nrf2-mediated induction of stressinduced antioxidant and phase II detoxification enzymes in BEAS-2B human bronchial epithelial cells [434]. Similar results were obtained with aldehyde carotenoid derivatives [432].

\subsubsection{Suppressors of the NF- $\kappa B$ pathway}

Carotenoids and carotenoid derivatives interact with the NF-KB pathway to suppress NF$\kappa B$ activation, thereby inhibiting the downstream production of inflammatory cytokines and disturbing NF-KB-promoted cancer development $[415,435]$. NF- $\kappa B$, similar to Nrf2, is normally bound to a cytoplasmic inhibitor ( $1 \mathrm{~KB})$ from which it dissociates in response to a variety of stress stimuli (e.g. cytokines, free radicals, heavy metals, ultraviolet irradiation, oxidized LDL, bacterial or viral antigens). This allows NF-KB to translocate to the nucleus to exert its transcriptional activity on inflammation-related genes. A possible unified explanation for the interference of carotenoids and/or carotenoid derivatives with the Nrf2 and NF-KB pathways (Figure 10) is that they induce a certain degree of intracellular oxidative stress, leading to the oxidative modulation of sulfhydryl groups or phosphorylation status of Nrf2, NF-KB or their interacting proteins [415, 422]. For instance, it has been reported that apocarotenals contain electrophilic groups that can

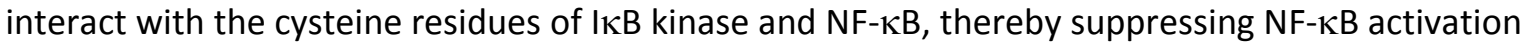
[415].

\subsubsection{Interaction with transcription factors}

The hydrophobic nature of carotenoids and their derivatives makes them good candidates for interaction with transcription factors of the NR superfamily (Figure 10), which bind ligands that easily transverse the cell membrane or lipophilic ligands generated intracellularly. Indeed, RA isomers derived from $\beta$-carotene (or preformed vitamin A) are endogenous agonist ligands of specific NRs collectively termed retinoid receptors: the canonical RA receptors (RARs: $\alpha, \beta$ and $\gamma$ ) and the retinoid X receptors (RXR: $\alpha, \beta$ and $\gamma$ ). The all-trans RA isomer (atRA) binds the RARs, whereas the 9-cis-RA isomer binds both the RARs and the RXRs. In a ligand-dependent manner, the retinoid receptors directly interact with the genome and modify transcription of specific genes, or interfere with the activity of other transcription factors such as activator protein-1 (AP-1) or CCAAT-enhancer binding proteins (C/EBPs) [321, 436]. Heterodimers of RAR with RXR control the expression of typical retinoid target genes in a manner that depends on atRA binding to the RAR moiety and subsequent recruitment of cofactor complexes to the gene promoter [436]. RXRs are obligate dimerization partners for many nuclear receptors besides the RARs, including peroxisome proliferator activated receptors (PPARs), liver $X$ receptor, farnesoid $X$ receptor, pregnane $X$ receptor, thyroid hormone receptor and vitamin $D$ receptor. Some RXR heterodimers respond to ligands of either partner and are synergistically activated when both ligands are bound, providing a mechanism for widespread effects of retinoids on gene expression [437]. Besides activating the canonical RARs, atRA (but not 9-cis-RA) might behave as an activating agonist ligand of PPAR $\beta / \delta$ but not the other PPAR isoforms, namely PPAR $\alpha$ and PPAR $\gamma$ [438], although conflicting results have been reported [439]. The intracellular RA binding proteins CRABP-II and FABP5 are thought to deliver atRA to RARs and PPAR $\beta / \delta$, respectively [440]. Effects of retinoids on cell differentiation and proliferation are largely downstream their interaction with the retinoid receptors [441]. For instance, repression of the pro-proliferative transcriptional activity of AP-1 by retinoid-bound RARs is key to the effects of retinoids against hyperproliferative diseases [441]. Retinoid interaction with lipid and energy metabolism in animals is also largely dependent on retinoid ability to activate NRs [321].

Other CCPs besides RA can interact with NR transcription factors to either promote or antagonize their action on target genes (Figure 10) [304, 432]. For example, apo-10'-lycopenoic acid, a product of eccentric cleavage of lycopene, inhibits lung cancer cell growth in vitro and suppresses lung tumorigenesis in a mouse model in vivo. Linked to this antiproliferative activity, apo-10'-lycopenoic acid transactivated the RAR $\beta$ promoter and induced the expression of RAR $\beta$, 
which led to the proposal that apo-10'-lycopenoic acid may function as a RA analogue [442]. Further, apo-10'-lycopenoic acid was demonstrated to reduce the production of proinflammatory markers in adipose tissue and adipocytes through RAR transactivation [443]. Other CCPs, such as $\beta$-apo-14'-carotenal, $\beta$-apo-14'-carotenoic acid and $\beta$-apo-13-carotenone (the three of them derived from eccentric cleavage of $\beta$-carotene), were shown to bind the RARs with high affinity and to antagonize the atRA-induced, RAR-dependent transactivation of target genes in cells of hepatic origin (HepG2) [444]. $\beta$-apo-14'-carotenal was also shown to repress agonist-induced activation of PPARs, leading to the inhibition of adipose conversion of preadipose cells, which is PPAR $\gamma$-dependent [445]. Because some of these CCPs are contained in foods and found in human plasma, it has been proposed they may act as naturally occurring retinoid antagonists [387].

Even intact carotenoid molecules appear to be able to interact physically with NRs. In vitro studies have suggested that $\beta$-cryptoxanthin can behave as a RAR agonist ligand [446] and astaxanthin as a PPAR $\gamma$ antagonist ligand [447]. RAR agonism and PPAR $\gamma$ antagonism are both expected to inhibit adipogenesis of preadipose cells. In agreement, both $\beta$-cryptoxanthin and astaxanthin have inhibitory effects on adipogenesis and anti-obesity activity in mouse models $[304,446,447]$.

\subsubsection{Other mechanisms}

There is evidence that retinoids may impact cell metabolism and cell-signaling pathways through extragenomic actions including retinoylation (acylation by RA) of proteins and activation of protein kinase cascades [321, 387, 415, 448-450]. It cannot be discarded that other carotenoid derivatives may act also in these ways.

\section{Conclusions and perspectives}

There is no doubt that our understanding of carotenoid biosynthesis and functions in microbes, plants and animals has greatly advanced in the last few years. New structural and regulatory genes have been identified, including those encoding ZISO, CCD enzymes responsible for the production of valuable pigments and volatiles, and other enzymes involved in strigolactone biosynthesis as well as transcriptional regulators of carotenoid biosynthesis in plants and bacteria. However, other biosynthetic steps (such as those involved in the isomerization and esterification of plant carotenoids or the formation of many microbial carotenoids) still remain elusive. New available multi-omics strategies should contribute to identify and characterize the genes and enzymes involved in these steps. Technologies are also available to discover new carotenoid-producing organisms from marine environments (such as archaea, bacteria, fungi, and phytoplankton) and to investigate the potential industrial applications of novel carotenoids that could be found or that are already known. Strikingly, the rules for the nomenclature of carotenoids were approved over 40 years ago and have hardly changed ever since. It appears necessary to revise and update the nomenclature rules to remedy possible problems such as those involving newly identified carotenoids with uncommon structures or those associated to acyl, sugar or other moieties. In relation to these latter carotenoids, it is important to consider that such associations do change considerably the properties of the "unmodified" carotenoid. Few studies on this respect exist despite the corresponding information might be very useful to open the range of practical applications of carotenoids. It is noteworthy that only a small fraction of the hundreds of carotenoids described so far are being studied in depth, which means that there is much knowledge to be gained into the properties, actions and practical applications of carotenoids.

Strigolactones have been consolidated as a new class of plant hormones, but information regarding tissue-specific functions, transport, and perception of the different strigolactones produced by plants remain mostly unknown. Besides strigolactones, many CCPs of unknown identity have been reported as having signalling functions in plant development and environmental interactions. Research on carotenoid-related signals is actually one of the hottest 
topics in the carotenoid field, not only in plants but also in animals and other organisms. However, progress is somewhat hindered for different reasons, including inconsistencies in their nomenclature (e.g. not all CCPs are apocarotenoids: ABA, strigolactones, or retinoids are not strictly apocarotenoids), scarcity of commercially available standards, and lack of extinction coefficients and absorption spectra. Future research should lead to the identification of the biologically active molecules and the characterization of their physicochemical properties and physiological functions. It would also be important to investigate their biosynthetic pathways and their compartmentalization, as well as the molecular mechanism implicated in their regulation. Similarly, more efforts should be made to understand the function and subcellular localization of carotenoids and their derived metabolites in non-photosynthetic microorganisms such as fungi.

The discovery of carotenoid biosynthetic genes of fungal origin in several groups of animals, on the other hand, has challenged the previous dogma that animals do not produce carotenoids de novo, providing further examples of horizontal gene transfer as a mechanism to produce "natural transgenic organisms" with acquired new functions (i.e. the capacity to synthesize carotenoids). In the field of "artificial transgenic organisms", the last years have witnessed major advances in carotenoid biotechnology in plants and bacteria. Proof of concept for the biotechnological overproduction of high added-value carotenoids such as astaxanthin has been achieved in many organisms, but our still limited knowledge of the endogenous regulatory networks influencing metabolic fluxes and interaction with related pathways is still a major drawback for obtaining commercially-relevant levels. For example, the paramount influence of light on the accumulation of plant carotenoids has been known for long time but the basic mechanisms by which light signals are transduced to eventually modulate the biosynthesis and accumulation of these important photoprotective pigments were only recently unveiled. In this context, it will be important to transfer and apply the information generated in model plants to agronomic crops. Available knowledge should also be useful to develop genetic markers for genomics-assisted breeding programs aimed to improve carotenoid content and composition in crops. Recovery of germplasm and traditional varieties (landraces) should further allow to understand carotenoid diversity and to identify genetic traits that determine carotenoid profiles, as well as the changes that occurred during domestication. Furthermore, the power of new technologies such as synthetic biology and genome editing (e.g. via CRISPR/Cas9) still awaits to be fully exploited in the field.

Recent discoveries have also highlighted that sequestration and storage are central determinants of final carotenoid levels in plants. However, very little is known about the suborganellar structures involved in the sequestration and storage of carotenoids as well as the signals and mechanisms underlying their differentiation. In particular, the storage of carotenoid esters (very abundant in some plant tissues) represents an unexplored aspect in carotenoid research. Since the physicochemical properties of carotenoid esters highly differ from those of the corresponding free carotenoids, it is likely that they are stored in different plastidial compartments. This hypothesis, however, awaits experimental validation. In any case, plastids are much more than mere reservoirs of carotenoids, as they have a central role in controlling the activity of biosynthetic enzymes (many of which appear to be associated to carotenoid-rich membranes and plastoglobules) and the carotenoid profile at both qualitative and quantitative levels. We still know very little about where the enzymes of the carotenoid pathway are localized within plastids. The proposal that these enzymes can interact to form metabolic channels (metabolons) should be further investigated, as it opens the possibility of alternative regulatory mechanisms involved in carotenoid biosynthesis that have not yet been fully explored.

Understanding the molecular mechanisms underlying plastid identity will be key to manipulate plant carotenoid contents in the future. To date, however, the Or chaperone is the only protein known to trigger the differentiation of carotenoid-accumulating plastids (chromoplasts). And even in this case, the functional roles of Or in plastids but also in the nucleus (where it has also been localized) remain far from clear. Future work should establish what is the specific molecular pathway connecting Or activity and chromoplast differentiation. Other 
chaperones (such as $\mathrm{ClpB3}$ ) and proteases (the Clp proteolytic complex) were recently shown to regulate the correct folding (and hence activity) and the stability of carotenoid biosynthetic enzymes. Besides coordinating carotenoid biosynthesis with the supply of their biosynthetic precursors, the Clp protease also appears to have a role in chromoplasts differentiation, further contributing to regulate the accumulation of carotenoids.

The type of carotenoid storage structures in plastids can strongly influence carotenoid bioavailability. The number of carotenoids eaten in a varied diet is much larger than generally shown in food composition tables, and the study of carotenoid bioavailability should be extended to include such low-abundant carotenoids as well as xanthophyll esters, as they are the most common native state for hydroxy-carotenoids in many fruits. This is particularly relevant in the current context of searching and incorporating novel natural sources of carotenoids in the diet, mainly focused on exploiting the biodiversity of tropical regions, oceans and poles, as well as the use of underutilized plant species, cultivars or landraces. Furthermore, many dietary factors, as well as host-related factors, have been found to influence carotenoid absorption and metabolism in animals. There is an urgent need for standardized protocols to assess bioaccessibility of carotenoids (as well as their esters and derived products) in food matrices and for establishing RDA or adequate intake for individual carotenoids commonly present in our diets, such as $\beta$ cryptoxanthin, lutein, zeaxanthin, and lycopene, among others. In the case of provitamin A carotenoids, assessment of their contribution to the dietary intake of vitamin A should be more clearly determined in light of the results of recent studies on bioavailability from different types of food matrices and chemical structures (carotene vs xanthophyll) and on the effect of polymorphisms in their conversion to vitamin $\mathrm{A}$.

The idea that carotenoids and their derived products have biological activities in humans other than provitamin A was also consolidated in the last years. The spectrum of health-related processes impacted by carotenoids and CCPs (including but not restricted to retinoids) in mammals is rapidly expanding. Besides the already known beneficial health effects of carotenoids for protection against photodamage, macular degeneration, and chronic diseases, new results are demonstrating a positive effect on preventing obesity and, particularly in the case of lutein, improving cognitive functions. Interaction with oxidative stress and inflammation pathways, and with NR-mediated signaling might explain many of these effects. However, the contribution of most dietary carotenoids (including those lacking color, such as phytoene and phytofluene) to human health remains virtually unknown. Whether the native compounds alone or rather their metabolites are responsible for the attributed health effects remains unclear. Furthermore, much is yet to be discovered regarding the regulated production and biological action of CCPs in animal tissues. Investigating the role and biological activities of catabolites produced from dietary carotenoids during digestion is expected to be a promising area of research for the near future. This is particularly important for carotenoids labelled as "nonabsorbed" and "non-bioavailable" such as violaxanthin and neoxanthin. Future research should also contribute to understand the biological mechanisms of action underlying the proposed health-related activities of carotenoids, eventually resulting in optimized suggestions for dietary intakes.

\section{Funding}

Authors belong to the Spanish Carotenoid Network (CaRed) funded by the Spanish MINECO (Ministry of Economy, Industry and Competitiveness) grant BIO2015-71703-REDT, the European Carotenoid Network (EuroCaroten) funded by the European Commission COST action CA15136, and the Ibero-American Carotenoid Network (IberCarot) funded by the Ibero-American Programme for Science, Technology and Development (CYTED) grant 112RT0445. Research on carotenoids at the authors' individual laboratories was supported by Spanish MINECO grants BIO2014-59092-P to MRC, BIO2015-69613-R to JA and MCL, AGL2013-43522-R to AB, BIO201677000-R to LGG, AGL2014-53195-R to DHM, AGL2015-67019-P to AP, BIO2014-54441-P to CZ, 
and AGL2015-70218 to LZ and MJR; Instituto de Salud Carlos III-FEDER grant PI16/01991 to BOA; Generalitat de Catalunya grants 2014SGR-1434 to MRC and AB and 2014SGR-1296 to CZ; and Junta de Andalucia grants P10-CTS-6638 to JA and P12-AGR-1287 to AJMM. We also acknowledge the financial support of the CERCA Programme of the Generalitat de Catalunya and the Severo Ochoa Programme for Centres of Excellence in R\&D 2016-2019 to CRAG (SEV-2015-0533).

\section{References}

[1] J. Yabuzaki, Carotenoids Database: structures, chemical fingerprints and distribution among organisms, Database (Oxford) 2017 (2017).

[2] G. Britton, Structure and properties of carotenoids in relation to function, FASEB J 9 (1995) 1551-8.

[3] G. Britton, S. Liaaen-Jensen, H. Pfander, , , . Carotenoids Birkhäuser, Basel, Switzerland, 2004.

[4] B.C.L. Weedon, G.P. Moss, Structure and nomenclature, in: G. Britton, S. Liaaen-Jensen, H. Pfander (Eds.), Carotenoids, Birkhauser, Basel, Switzerland, 1995, pp. 27-44.

[5] A.J. Melendez-Martinez, M. Paulino, C.M. Stinco, P. Mapelli-Brahm, X.D. Wang, Study of the time-course of cis/trans (Z/E) isomerization of lycopene, phytoene, and phytofluene from tomato, J Agric Food Chem 62 (2014) 12399-406.

[6] G.A. Chasse, M.L. Mak, E. Deretey, I. Farkas, L.L. Torday, J.G. Papp, D.S.R. Sarma, A. Agarwal, S. Chakravarthi, S. Agarwal, A.V. Rao, An ab initio computational study on selected lycopene isomers, Journal of Molecular Structure 571 (2001) 27-37.

[7] D.B. Rodriguez-Amaya, Changes in carotenoids during processing and storage of foods, Arch Latinoam Nutr 49 (1999) 38S-47S.

[8] A. Schieber, R. Carle, Occurrence of carotenoid cis-isomers in food: Technological, analytical, and nutritional implications, Trends Food Sci. Technol. 16 (2005) 416-422.

[9] A. Than, P.M. Bramley, B.H. Davies, A.F. Rees, Stereochemistry of phytoene, Phytochemistry 11 (1972) 3187-3192.

[10] T. Isaacson, G. Ronen, D. Zamir, J. Hirschberg, Cloning of tangerine from tomato reveals a carotenoid isomerase essential for the production of beta-carotene and xanthophylls in plants, Plant Cell 14 (2002) 333-342.

[11] H.H. Schmitz, C. Emenhiser, S.J. Schwartz, HPLC separation of geometric carotene isomers using a calcium hydroxide stationary phase, Journal of Agricultural and Food Chemistry 43 (1995) 1212-1218.

[12] C.J. Xu, P.D. Fraser, W.J. Wang, P.M. Bramley, Differences in the carotenoid content of ordinary citrus and lycopene-accumulating mutants, J Agric Food Chem 54 (2006) 5474-81.

[13] A.J. Meléndez-Martínez, I.M. Vicario, F.J. Heredia, Geometrical isomers of violaxanthin in orange juice, Food Chemistry 104 (2007) 169-175.

[14] A.J. Meléndez-Martínez, G. Britton, I.M. Vicario, F.J. Heredia, Identification of isolutein (lutein epoxide) as cis-antheraxanthin in orange juice, Journal of Agricultural and Food Chemistry 53 (2005) 9369-9373.

[15] V. Bohm, N.L. Puspitasari-Nienaber, M.G. Ferruzzi, S.J. Schwartz, Trolox equivalent antioxidant capacity of different geometrical isomers of alpha-carotene, beta-carotene, lycopene, and zeaxanthin, J Agric Food Chem 50 (2002) 221-6.

[16] W. Stahl, W. Schwarz, A.R. Sundquist, H. Sies, cis-trans isomers of lycopene and betacarotene in human serum and tissues, Arch Biochem Biophys 294 (1992) 173-7. 
[17] A.J. Meléndez-Martínez, G. Britton, I.M. Vicario, F.J. Heredia, The complex carotenoid pattern of orange juices from concentrate, Food Chemistry 109 (2008) 546-553.

[18] R.A. Bone, J.T. Landrum, G.W. Hime, A. Cains, J. Zamor, Stereochemistry of the human macular carotenoids, Invest Ophthalmol Vis Sci 34 (1993) 2033-40.

[19] H.M. Rasmussen, T. Muzhingi, E.M.R. Eggert, E.J. Johnson, Lutein, zeaxanthin, mesozeaxanthin content in egg yolk and their absence in fish and seafood, Journal of Food Composition and Analysis 27 (2012) 139-144.

[20] E.J. Johnson, M. Neuringer, R.M. Russell, W. Schalch, D.M. Snodderly, Nutritional manipulation of primate retinas, III: Effects of lutein or zeaxanthin supplementation on adipose tissue and retina of xanthophyll-free monkeys, Invest Ophthalmol Vis Sci 46 (2005) 692-702.

[21] M.I. Mínguez-Mosquera, D. Hornero-Méndez, Changes in Carotenoid Esterification during the Fruit Ripening of Capsicum annuum Cv. Bola, Journal of Agricultural and Food Chemistry 42 (1994) 640-644.

[22] R. Delgado-Pelayo, D. Hornero-Méndez, Identification and quantitative analysis of carotenoids and their esters from sarsaparilla (Smilax aspera L.) berries, Journal of Agricultural and Food Chemistry 60 (2012) 8225-8232.

[23] E. Murillo, D. Giuffrida, D. Menchaca, P. Dugo, G. Torre, A.J. Meléndez-Martinez, L. Mondello, Native carotenoids composition of some tropical fruits, Food Chemistry 140 (2013) 825-836.

[24] A.Z. Mercadante, D.B. Rodrigues, F.C. Petry, L.R.B. Mariutti, Carotenoid esters in foods - A review and practical directions on analysis and occurrence, Food Res Int 99 (2017) 830-850.

[25] D.E. Breithaupt, U. Wirt, A. Bamedi, Differentiation between lutein monoester regioisomers and detection of lutein diesters from marigold flowers (Tagetes erecta L.) and Several fruits by liquid chromatography-mass spectrometry, Journal of Agricultural and Food Chemistry 50 (2002) 66-70.

[26] D.E. Breitbaupt, A. Bamedi, Carotenoids and carotenoid esters in potatoes (Solanum tuberosum L.): New insights into an ancient vegetable, Journal of Agricultural and Food Chemistry 50 (2002) 7175-7181.

[27] S. Pfister, P. Meyer, A. Steck, H. Pfander, Isolation and structure elucidation of carotenoidglycosyl esters in gardenia fruits (Gardenia jasminoides Ellis) and saffron (Crocus sativus Linne), Journal of Agricultural and Food Chemistry 44 (1996) 2612-2615.

[28] P. Bhosale, P.S. Bernstein, Vertebrate and invertebrate carotenoid-binding proteins, 2007, pp. 121-127.

[29] F. Shahidi, Metusalach, J.A. Brown, Carotenoid pigments in seafoods and aquaculture, Critical Reviews in Food Science and Nutrition 38 (1998) 1-67.

[30] D. Hoischen, L.U. Colmenares, J. Liu, C.J. Simmons, G. Britton, R.S.H. Liu, Fluorinated analogs of the carotenoprotein, $\alpha$-crustacyanin, Bioorganic Chemistry 26 (1998) 365-374.

[31] A.J. Melendez-Martinez, P. Mapelli-Brahm, A. Benitez-Gonzalez, C.M. Stinco, A comprehensive review on the colorless carotenoids phytoene and phytofluene, Arch Biochem Biophys 572 (2015) 188-200.

[32] A.J. Meléndez-Martínez, M. Paulino, C.M. Stinco, P. Mapelli-Brahm, X.D. Wang, Study of the time-course of cis/trans (Z/E) isomerization of lycopene, phytoene, and phytofluene from tomato, Journal of Agricultural and Food Chemistry 62 (2014) 12399-12406. 
[33] A. Martínez, C.M. Stinco, A.J. Meléndez-Martinez, Free radical scavenging properties of phytofluene and phytoene isomers as compared to Lycopene: a combined experimental and theoretical study, Journal of Physical Chemistry B 118 (2014) 9819-9825.

[34] A. Pokhilko, J. Bou-Torrent, P. Pulido, M. Rodriguez-Concepcion, O. Ebenhoh, Mathematical modelling of the diurnal regulation of the MEP pathway in Arabidopsis, New Phytol 206 (2015) 1075-85.

[35] J. Penuelas, S. Munne-Bosch, Isoprenoids: an evolutionary pool for photoprotection, Trends Plant Sci 10 (2005) 166-9.

[36] L. Leonelli, M.D. Brooks, K.K. Niyogi, Engineering the lutein epoxide cycle into Arabidopsis thaliana, Proc Natl Acad Sci U S A 114 (2017) E7002-E7008.

[37] J.I. Garcia-Plazaola, R. Esteban, B. Fernandez-Marin, I. Kranner, A. Porcar-Castell, Thermal energy dissipation and xanthophyll cycles beyond the Arabidopsis model, Photosynth Res 113 (2012) 89-103.

[38] R. Esteban, J.M. Becerril, J.I. Garcia-Plazaola, Lutein epoxide cycle, more than just a forest tale, Plant Signal Behav 4 (2009) 342-4.

[39] H. Hashimoto, C. Uragami, R.J. Cogdell, Carotenoids and Photosynthesis, Subcell Biochem 79 (2016) 111-39.

[40] X. Hou, J. Rivers, P. Leon, R.P. McQuinn, B.J. Pogson, Synthesis and Function of Apocarotenoid Signals in Plants, Trends Plant Sci 21 (2016) 792-803.

[41] B. Llorente, J.F. Martinez-Garcia, C. Stange, M. Rodriguez-Concepcion, Illuminating colors: regulation of carotenoid biosynthesis and accumulation by light, Curr Opin Plant Biol 37 (2017) 49-55.

[42] G. Britton, Functions of Intact Carotenoids, in: G. Britton, S. Liaaen-Jensen, H. Pfander (Eds.), Carotenoids, Vol 4: Natural Functions, Birkhäuser Verlag, Basel, Switzerland, 2008, pp. 189212.

[43] M.A. Ruiz-Sola, M. Rodriguez-Concepcion, Carotenoid biosynthesis in Arabidopsis: a colorful pathway, Arabidopsis Book 10 (2012) e0158.

[44] N. Nisar, L. Li, S. Lu, N.C. Khin, B.J. Pogson, Carotenoid metabolism in plants, Mol Plant 8 (2015) 68-82.

[45] A.R. Moise, S. Al-Babili, E.T. Wurtzel, Mechanistic aspects of carotenoid biosynthesis, Chem Rev 114 (2014) 164-93.

[46] M. Rodriguez-Concepcion, A. Boronat, Breaking new ground in the regulation of the early steps of plant isoprenoid biosynthesis, Curr Opin Plant Biol 25 (2015) 17-22.

[47] L.P. Wright, J.M. Rohwer, A. Ghirardo, A. Hammerbacher, M. Ortiz-Alcaide, B. Raguschke, J.P. Schnitzler, J. Gershenzon, M.A. Phillips, Deoxyxylulose 5-Phosphate Synthase Controls Flux through the Methylerythritol 4-Phosphate Pathway in Arabidopsis, Plant Physiol 165 (2014) 1488-1504.

[48] A. Hemmerlin, Post-translational events and modifications regulating plant enzymes involved in isoprenoid precursor biosynthesis, Plant Sci 203-204 (2013) 41-54.

[49] U. Flores-Pérez, S. Sauret-Güeto, E. Gas, P. Jarvis, M. Rodríguez-Concepción, A Mutant Impaired in the Production of Plastome-Encoded Proteins Uncovers a Mechanism for the Homeostasis of Isoprenoid Biosynthetic Enzymes in Arabidopsis Plastids, Plant Cell 20 (2008) 1303-1315. 
[50] P. Pulido, E. Llamas, B. Llorente, S. Ventura, L.P. Wright, M. Rodriguez-Concepcion, Specific Hsp100 Chaperones Determine the Fate of the First Enzyme of the Plastidial Isoprenoid Pathway for Either Refolding or Degradation by the Stromal Clp Protease in Arabidopsis, PLoS Genet 12 (2016) e1005824.

[51] P. Pulido, G. Toledo-Ortiz, M.A. Phillips, L.P. Wright, M. Rodriguez-Concepcion, Arabidopsis Jprotein $\mathrm{J} 20$ delivers the first enzyme of the plastidial isoprenoid pathway to protein quality control, Plant Cell 25 (2013) 4183-94.

[52] E. Llamas, P. Pulido, M. Rodriguez-Concepcion, Interference with plastome gene expression and Clp protease activity in Arabidopsis triggers a chloroplast unfolded protein response to restore protein homeostasis, PLoS Genet 13 (2017) e1007022.

[53] A. Ghirardo, L.P. Wright, Z. Bi, M. Rosenkranz, P. Pulido, M. Rodriguez-Concepcion, U. Niinemets, N. Bruggemann, J. Gershenzon, J.P. Schnitzler, Metabolic flux analysis of plastidic isoprenoid biosynthesis in poplar leaves emitting and nonemitting isoprene, Plant Physiol 165 (2014) 37-51.

[54] S. Sauret-Gueto, P. Botella-Pavia, U. Flores-Perez, J.F. Martinez-Garcia, C. San Roman, P. Leon, A. Boronat, M. Rodriguez-Concepcion, Plastid cues posttranscriptionally regulate the accumulation of key enzymes of the methylerythritol phosphate pathway in Arabidopsis, Plant Physiol 141 (2006) 75-84.

[55] M.A. Ruiz-Sola, D. Coman, G. Beck, M.V. Barja, M. Colinas, A. Graf, R. Welsch, P. Rutimann, P. Buhlmann, L. Bigler, W. Gruissem, M. Rodriguez-Concepcion, E. Vranova, Arabidopsis GERANYLGERANYL DIPHOSPHATE SYNTHASE 11 is a hub isozyme required for the production of most photosynthesis-related isoprenoids, New Phytol 209 (2016) 252-64.

[56] M.A. Ruiz-Sola, M.V. Barja, D. Manzano, B. Llorente, B. Schipper, J. Beekwilder, M. Rodriguez-Concepcion, A Single Arabidopsis Gene Encodes Two Differentially Targeted Geranylgeranyl Diphosphate Synthase Isoforms, Plant Physiol 172 (2016) 1393-1402.

[57] F. Zhou, C.Y. Wang, M. Gutensohn, L. Jiang, P. Zhang, D. Zhang, N. Dudareva, S. Lu, A recruiting protein of geranylgeranyl diphosphate synthase controls metabolic flux toward chlorophyll biosynthesis in rice, Proc Natl Acad Sci U S A 114 (2017) 6866-6871.

[58] J. Ji, G. Wang, J. Wang, P. Wang, Functional analysis of multiple carotenogenic genes from Lycium barbarum and Gentiana lutea L. for their effects on beta-carotene production in transgenic tobacco, Biotechnol Lett 31 (2009) 305-12.

[59] W. Chen, S. He, D. Liu, G.B. Patil, H. Zhai, F. Wang, T.J. Stephenson, Y. Wang, B. Wang, B. Valliyodan, H.T. Nguyen, Q. Liu, A Sweetpotato Geranylgeranyl Pyrophosphate Synthase Gene, IbGGPS, Increases Carotenoid Content and Enhances Osmotic Stress Tolerance in Arabidopsis thaliana, PLoS One 10 (2015) e0137623.

[60] S.K. Tata, J. Jung, Y.H. Kim, J.Y. Choi, J.Y. Jung, I.J. Lee, J.S. Shin, S.B. Ryu, Heterologous expression of chloroplast-localized geranylgeranyl pyrophosphate synthase confers fast plant growth, early flowering and increased seed yield, Plant Biotechnol J 14 (2016) 29-39.

[61] P.D. Fraser, S. Romer, C.A. Shipton, P.B. Mills, J.W. Kiano, N. Misawa, R.G. Drake, W. Schuch, P.M. Bramley, Evaluation of transgenic tomato plants expressing an additional phytoene synthase in a fruit-specific manner, Proc Natl Acad Sci U S A 99 (2002) 1092-7.

[62] L. Liu, Z. Shao, M. Zhang, Q. Wang, Regulation of carotenoid metabolism in tomato, Mol Plant 8 (2015) 28-39.

[63] M. Shumskaya, E.T. Wurtzel, The carotenoid biosynthetic pathway: thinking in all dimensions, Plant Sci 208 (2013) 58-63. 
[64] M. Shumskaya, L.M. Bradbury, R.R. Monaco, E.T. Wurtzel, Plastid localization of the key carotenoid enzyme phytoene synthase is altered by isozyme, allelic variation, and activity, Plant Cell 24 (2012) 3725-41.

[65] R. Welsch, X. Zhou, H. Yuan, D. Alvarez, T. Sun, D. Schlossarek, Y. Yang, G. Shen, H. Zhang, M. Rodriguez-Concepcion, T.W. Thannhauser, L. Li, Clp Protease and OR Directly Control the Proteostasis of Phytoene Synthase, the Crucial Enzyme for Carotenoid Biosynthesis in Arabidopsis, Mol Plant (2017).

[66] L. D’Andrea, M. Simon-Moya, B. Llorente, E. Llamas, M. Marro, P. Loza-Alvarez, L. Li, M. Rodriguez-Concepcion, Interference with Clp protease impairs carotenoid accumulation during tomato fruit ripening, Journal of Experimental Botany in press (2018).

[67] X. Zhou, R. Welsch, Y. Yang, D. Alvarez, M. Riediger, H. Yuan, T. Fish, J. Liu, T.W. Thannhauser, L. Li, Arabidopsis OR proteins are the major posttranscriptional regulators of phytoene synthase in controlling carotenoid biosynthesis, Proc Natl Acad Sci U S A 112 (2015) 3558-63.

[68] S. Park, H.S. Kim, Y.J. Jung, S.H. Kim, C.Y. Ji, Z. Wang, J.C. Jeong, H.S. Lee, S.Y. Lee, S.S. Kwak, Orange protein has a role in phytoene synthase stabilization in sweetpotato, Sci Rep 6 (2016) 33563.

[69] Z. Luo, J. Zhang, J. Li, C. Yang, T. Wang, B. Ouyang, H. Li, J. Giovannoni, Z. Ye, A STAY-GREEN protein SISGR1 regulates lycopene and beta-carotene accumulation by interacting directly with SIPSY1 during ripening processes in tomato, New Phytol 198 (2013) 442-52.

[70] J. Arango, M. Jourdan, E. Geoffriau, P. Beyer, R. Welsch, Carotene Hydroxylase Activity Determines the Levels of Both alpha-Carotene and Total Carotenoids in Orange Carrots, Plant Cell 26 (2014) 2223-2233.

[71] D.E. Kachanovsky, S. Filler, T. Isaacson, J. Hirschberg, Epistasis in tomato color mutations involves regulation of phytoene synthase 1 expression by cis-carotenoids, Proc Natl Acad Sci U S A 109 (2012) 19021-6.

[72] Y. Chen, F. Li, E.T. Wurtzel, Isolation and characterization of the Z-ISO gene encoding a missing component of carotenoid biosynthesis in plants, Plant Physiol 153 (2010) 66-79.

[73] R. Li, C. Kang, X. Song, L. Yu, D. Liu, S. He, H. Zhai, Q. Liu, A zeta-carotene desaturase gene, IbZDS, increases beta-carotene and lutein contents and enhances salt tolerance in transgenic sweetpotato, Plant Sci 262 (2017) 39-51.

[74] R.P. McQuinn, B. Wong, J.J. Giovannoni, AtPDS overexpression in tomato: exposing unique patterns of carotenoid self-regulation and an alternative strategy for the enhancement of fruit carotenoid content, Plant Biotechnol J (2017).

[75] M. Renato, I. Pateraki, A. Boronat, J. Azcon-Bieto, Tomato fruit chromoplasts behave as respiratory bioenergetic organelles during ripening, Plant Physiol 166 (2014) 920-33.

[76] W.J. Nawrocki, N.J. Tourasse, A. Taly, F. Rappaport, F.A. Wollman, The plastid terminal oxidase: its elusive function points to multiple contributions to plastid physiology, Annu Rev Plant Biol 66 (2015) 49-74.

[77] M. Renato, A. Boronat, J. Azcon-Bieto, Respiratory processes in non-photosynthetic plastids, Front Plant Sci 6 (2015) 496.

[78] J. Beltran, B. Kloss, J.P. Hosler, J. Geng, A. Liu, A. Modi, J.H. Dawson, M. Sono, M. Shumskaya, C. Ampomah-Dwamena, J.D. Love, E.T. Wurtzel, Control of carotenoid biosynthesis through a heme-based cis-trans isomerase, Nat Chem Biol 11 (2015) 598-605. 
[79] C.I. Cazzonelli, B.J. Pogson, Source to sink: regulation of carotenoid biosynthesis in plants, Trends Plant Sci 15 (2010) 266-74.

[80] J. Kim, J.J. Smith, L. Tian, D. Dellapenna, The evolution and function of carotenoid hydroxylases in Arabidopsis, Plant Cell Physiol 50 (2009) 463-79.

[81] E. Mellado-Ortega, D. Hornero-Mendez, Isolation and identification of lutein esters, including their regioisomers, in tritordeum (xTritordeum Ascherson et Graebner) grains: Evidence for a preferential xanthophyll acyltransferase activity, Food Chem 135 (2012) 1344-52.

[82] R. Fernandez-Orozco, L. Gallardo-Guerrero, D. Hornero-Mendez, Carotenoid profiling in tubers of different potato (Solanum sp) cultivars: accumulation of carotenoids mediated by xanthophyll esterification, Food Chem 141 (2013) 2864-72.

[83] K.J. van Wijk, F. Kessler, Plastoglobuli: Plastid Microcompartments with Integrated Functions in Metabolism, Plastid Developmental Transitions, and Environmental Adaptation, Annu Rev Plant Biol 68 (2017) 253-289.

[84] T. Ariizumi, S. Kishimoto, R. Kakami, T. Maoka, H. Hirakawa, Y. Suzuki, Y. Ozeki, K. Shirasawa, S. Bernillon, Y. Okabe, A. Moing, E. Asamizu, C. Rothan, A. Ohmiya, H. Ezura, Identification of the carotenoid modifying gene PALE YELLOW PETAL 1 as an essential factor in xanthophyll esterification and yellow flower pigmentation in tomato (Solanum lycopersicum), Plant J 79 (2014) 453-65.

[85] F.X. Cunningham, E. Gantt, Genes and enzymes of carotenoid biosynthesis in plants, Ann Rev Plant Physiol Plant Mol Biol 49 (1998) 557-583.

[86] A.B. Lopez, Y. Yang, T.W. Thannhauser, L. Li, Phytoene desaturase is present in a large protein complex in the plastid membrane, Physiol Plant 133 (2008) 190-8.

[87] A.J. Ytterberg, J.B. Peltier, K.J. van Wijk, Protein profiling of plastoglobules in chloroplasts and chromoplasts. A surprising site for differential accumulation of metabolic enzymes, Plant Physiol 140 (2006) 984-97.

[88] C. Barsan, M. Zouine, E. Maza, W. Bian, I. Egea, M. Rossignol, D. Bouyssie, C. Pichereaux, E. Purgatto, M. Bouzayen, A. Latche, J.C. Pech, Proteomic analysis of chloroplast-to-chromoplast transition in tomato reveals metabolic shifts coupled with disrupted thylakoid biogenesis machinery and elevated energy-production components, Plant Physiol 160 (2012) 708-25.

[89] E.I. Hallin, M. Hasan, K. Guo, H.E. Akerlund, Molecular studies on structural changes and oligomerisation of violaxanthin de-epoxidase associated with the $\mathrm{pH}$-dependent activation, Photosynth Res 129 (2016) 29-41.

[90] J. Joyard, M. Ferro, C. Masselon, D. Seigneurin-Berny, D. Salvi, J. Garin, N. Rolland, Chloroplast proteomics and the compartmentation of plastidial isoprenoid biosynthetic pathways, Mol Plant 2 (2009) 1154-80.

[91] R. Welsch, P. Beyer, P. Hugueney, H. Kleinig, J. von Lintig, Regulation and activation of phytoene synthase, a key enzyme in carotenoid biosynthesis, during photomorphogenesis, Planta 211 (2000) 846-54.

[92] N. Galpaz, G. Ronen, Z. Khalfa, D. Zamir, J. Hirschberg, A chromoplast-specific carotenoid biosynthesis pathway is revealed by cloning of the tomato white-flower locus, Plant Cell 18 (2006) 1947-60.

[93] P.D. Fraser, W. Schuch, P.M. Bramley, Phytoene synthase from tomato (Lycopersicon esculentum) chloroplasts--partial purification and biochemical properties, Planta 211 (2000) 361-9. 
[94] M. Nogueira, L. Mora, E.M. Enfissi, P.M. Bramley, P.D. Fraser, Subchromoplast sequestration of carotenoids affects regulatory mechanisms in tomato lines expressing different carotenoid gene combinations, Plant Cell 25 (2013) 4560-79.

[95] L. Li, H. Yuan, Y. Zeng, Q. Xu, Plastids and Carotenoid Accumulation, Subcell Biochem 79 (2016) 273-93.

[96] M. Liebers, B. Grubler, F. Chevalier, S. Lerbs-Mache, L. Merendino, R. Blanvillain, T. Pfannschmidt, Regulatory Shifts in Plastid Transcription Play a Key Role in Morphological Conversions of Plastids during Plant Development, Front Plant Sci 8 (2017) 23.

[97] C.A. Howitt, B.J. Pogson, Carotenoid accumulation and function in seeds and non-green tissues., Plant Cell Environ. 29 (2006) 435-445.

[98] K. Solymosi, B. Schoefs, Etioplast and etio-chloroplast formation under natural conditions: the dark side of chlorophyll biosynthesis in angiosperms, Photosynth Res 105 (2010) 143-66.

[99] A. Rodriguez-Villalon, E. Gas, M. Rodriguez-Concepcion, Phytoene synthase activity controls the biosynthesis of carotenoids and the supply of their metabolic precursors in dark-grown Arabidopsis seedlings, Plant J 60 (2009) 424-435.

[100] H. Yuan, K. Owsiany, T.E. Sheeja, X. Zhou, C. Rodriguez, Y. Li, R. Welsch, N. Chayut, Y. Yang, T.W. Thannhauser, M.V. Parthasarathy, Q. Xu, X. Deng, Z. Fei, A. Schaffer, N. Katzir, J. Burger, Y. Tadmor, L. Li, A Single Amino Acid Substitution in an ORANGE Protein Promotes Carotenoid Overaccumulation in Arabidopsis, Plant Physiol 169 (2015) 421-31.

[101] I. Egea, C. Barsan, W. Bian, E. Purgatto, A. Latche, C. Chervin, M. Bouzayen, J.C. Pech, Chromoplast differentiation: current status and perspectives, Plant Cell Physiol 51 (2010) 1601-11.

[102] T. Sun, H. Yuan, H. Cao, M. Yazdani, Y. Tadmor, L. Li, Carotenoid Metabolism in Plants: The Role of Plastids, Mol Plant (2017).

[103] R.M. Schweiggert, R. Carle, Carotenoid deposition in plant and animal foods and its impact on bioavailability, Crit Rev Food Sci Nutr 57 (2017) 1807-1830.

[104] J. Lado, L. Zacarias, A. Gurrea, A. Page, A. Stead, M.J. Rodrigo, Exploring the diversity in Citrus fruit colouration to decipher the relationship between plastid ultrastructure and carotenoid composition, Planta 242 (2015) 645-61.

[105] J. Hempel, E. Amrehn, S. Quesada, P. Esquivel, V.M. Jimenez, A. Heller, R. Carle, R.M. Schweiggert, Lipid-dissolved gamma-carotene, beta-carotene, and lycopene in globular chromoplasts of peach palm (Bactris gasipaes Kunth) fruits, Planta 240 (2014) 1037-50.

[106] P. Sitte, H. Falk, B. Liedvogel, Chromoplasts, in: F.G.C. Czygan (Ed.), Pigments in Plants, G Fischer Verl, Stuttgart, New York, 1980, pp. 117-148.

[107] J. Gross, Pigments in fruits, in: B.S. Schweigert (Ed.), Food science and technology: a series of monographs, London Academic Press1987.

[108] L. Li, D.J. Paolillo, M.V. Parthasarathy, E.M. Dimuzio, D.F. Garvin, A novel gene mutation that confers abnormal patterns of beta-carotene accumulation in cauliflower (Brassica oleracea var. botrytis), Plant J 26 (2001) 59-67.

[109] S. Lu, J. Van Eck, X. Zhou, A.B. Lopez, D.M. O'Halloran, K.M. Cosman, B.J. Conlin, D.J. Paolillo, D.F. Garvin, J. Vrebalov, L.V. Kochian, H. Kupper, E.D. Earle, J. Cao, L. Li, The cauliflower Or gene encodes a DnaJ cysteine-rich domain-containing protein that mediates high levels of beta-carotene accumulation, Plant Cell 18 (2006) 3594-605. 
[110] A.B. Lopez, J. Van Eck, B.J. Conlin, D.J. Paolillo, J. O'Neill, L. Li, Effect of the cauliflower Or transgene on carotenoid accumulation and chromoplast formation in transgenic potato tubers, J Exp Bot 59 (2008) 213-23.

[111] C. Bai, S.M. Rivera, V. Medina, R. Alves, E. Vilaprinyo, A. Sorribas, R. Canela, T. Capell, G. Sandmann, P. Christou, C. Zhu, An in vitro system for the rapid functional characterization of genes involved in carotenoid biosynthesis and accumulation, Plant J 77 (2014) 464-75.

[112] C. Bai, T. Capell, J. Berman, V. Medina, G. Sandmann, P. Christou, C. Zhu, Bottlenecks in carotenoid biosynthesis and accumulation in rice endosperm are influenced by the precursorproduct balance, Plant Biotechnol J 14 (2016) 195-205.

[113] J. Berman, U. Zorrilla-Lopez, V. Medina, G. Farre, G. Sandmann, T. Capell, P. Christou, C. Zhu, The Arabidopsis ORANGE (AtOR) gene promotes carotenoid accumulation in transgenic corn hybrids derived from parental lines with limited carotenoid pools, Plant Cell Rep 36 (2017) 933-945.

[114] N. Chayut, H. Yuan, S. Ohali, A. Meir, U. Sa'ar, G. Tzuri, Y. Zheng, M. Mazourek, S. Gepstein, X. Zhou, V. Portnoy, E. Lewinsohn, A.A. Schaffer, N. Katzir, Z. Fei, R. Welsch, L. Li, J. Burger, Y. Tadmor, Distinct Mechanisms of the ORANGE Protein in Controlling Carotenoid Flux, Plant Physiol 173 (2017) 376-389.

[115] H.V. Kilambi, R. Kumar, R. Sharma, Y. Sreelakshmi, Chromoplast-specific carotenoidassociated protein appears to be important for enhanced accumulation of carotenoids in hp1 tomato fruits, Plant Physiol 161 (2013) 2085-101.

[116] J. Kilcrease, L. Rodriguez-Uribe, R.D. Richins, J.M. Arcos, J. Victorino, M.A. O'Connell, Correlations of carotenoid content and transcript abundances for fibrillin and carotenogenic enzymes in Capsicum annum fruit pericarp, Plant Sci 232 (2015) 57-66.

[117] L.N. Nupur, A. Vats, S.K. Dhanda, G.P. Raghava, A.K. Pinnaka, A. Kumar, ProCarDB: a database of bacterial carotenoids, BMC Microbiol 16 (2016) 96.

[118] C.W. Chen, S.H. Hsu, M.T. Lin, Y.H. Hsu, Mass production of C50 carotenoids by Haloferax mediterranei in using extruded rice bran and starch under optimal conductivity of brined medium, Bioprocess Biosyst Eng 38 (2015) 2361-7.

[119] M. Rodrigo-Banos, I. Garbayo, C. Vilchez, M.J. Bonete, R.M. Martinez-Espinosa, Carotenoids from Haloarchaea and Their Potential in Biotechnology, Mar Drugs 13 (2015) 5508-32.

[120] M. Kelly, S. Norgard, S. Liaaen-Jensen, Bacterial carotenoids. 31. C50-carotenoids 5. Carotenoids of Halobacterium salinarium, especially bacterioruberin, Acta Chem Scand 24 (1970) 2169-82.

[121] F. Mandelli, V.S. Miranda, E. Rodrigues, A.Z. Mercadante, Identification of carotenoids with high antioxidant capacity produced by extremophile microorganisms, World J Microbiol Biotechnol 28 (2012) 1781-90.

[122] R. Calegari-Santos, R.A. Diogo, J.D. Fontana, T.M. Bonfim, Carotenoid Production by Halophilic Archaea Under Different Culture Conditions, Curr Microbiol 72 (2016) 641-51.

[123] A. Boronat, M. Rodriguez-Concepcion, Terpenoid biosynthesis in prokaryotes, Adv Biochem Eng Biotechnol 148 (2015) 3-18.

[124] B.S. Hundle, P. Beyer, H. Kleinig, G. Englert, J.E. Hearst, Carotenoids of Erwinia herbicola and an Escherichia coli HB101 strain carrying the Erwinia herbicola carotenoid gene cluster, Photochem Photobiol 54 (1991) 89-93.

[125] G.A. Armstrong, Eubacteria show their true colors: genetics of carotenoid pigment biosynthesis from microbes to plants, J Bacteriol 176 (1994) 4795-802. 
[126] G.A. Armstrong, J.E. Hearst, Carotenoids 2: Genetics and molecular biology of carotenoid pigment biosynthesis, FASEB J 10 (1996) 228-37.

[127] R.M. Schweiggert, R. Carle, Carotenoid production by bacteria, microalgae, and fungi, in: A. Kaczor, M. Baranska (Eds.), Carotenoids: Nutrition, Analysis and Technology, John Wiley \& Sons, Chichester, UK, 2016.

[128] N. Misawa, M. Nakagawa, K. Kobayashi, S. Yamano, Y. Izawa, K. Nakamura, K. Harashima, Elucidation of the Erwinia uredovora carotenoid biosynthetic pathway by functional analysis of gene products expressed in Escherichia coli, J Bacteriol 172 (1990) 6704-12.

[129] $\mathrm{H}$. Takano, The regulatory mechanism underlying light-inducible production of carotenoids in nonphototrophic bacteria, Biosci Biotechnol Biochem 80 (2016) 1264-73.

[130] L. Pasamontes, D. Hug, M. Tessier, H.P. Hohmann, J. Schierle, A.P. van Loon, Isolation and characterization of the carotenoid biosynthesis genes of Flavobacterium sp. strain R1534, Gene 185 (1997) 35-41.

[131] M. Elias-Arnanz, S. Padmanabhan, F.J. Murillo, Light-dependent gene regulation in nonphototrophic bacteria, Curr Opin Microbiol 14 (2011) 128-35.

[132] M. Galbis-Martinez, S. Padmanabhan, F.J. Murillo, M. Elias-Arnanz, CarF mediates signaling by singlet oxygen, generated via photoexcited protoporphyrin IX, in Myxococcus xanthus lightinduced carotenogenesis, J Bacteriol 194 (2012) 1427-36.

[133] G.A. Armstrong, Genetics of eubacterial carotenoid biosynthesis: a colorful tale, Annu Rev Microbiol 51 (1997) 629-59.

[134] B. Tian, Z. Xu, Z. Sun, J. Lin, Y. Hua, Evaluation of the antioxidant effects of carotenoids from Deinococcus radiodurans through targeted mutagenesis, chemiluminescence, and DNA damage analyses, Biochim Biophys Acta 1770 (2007) 902-11.

[135] L. Zhang, Q. Yang, X. Luo, C. Fang, Q. Zhang, Y. Tang, Knockout of crtB or crtl gene blocks the carotenoid biosynthetic pathway in Deinococcus radiodurans R1 and influences its resistance to oxidative DNA-damaging agents due to change of free radicals scavenging ability, Arch Microbiol 188 (2007) 411-9.

[136] P. Gao, J. Davies, R.Y.T. Kao, Dehydrosqualene Desaturase as a Novel Target for AntiVirulence Therapy against Staphylococcus aureus, MBio 8 (2017).

[137] G.Y. Liu, A. Essex, J.T. Buchanan, V. Datta, H.M. Hoffman, J.F. Bastian, J. Fierer, V. Nizet, Staphylococcus aureus golden pigment impairs neutrophil killing and promotes virulence through its antioxidant activity, J Exp Med 202 (2005) 209-15.

[138] S. Takaichi, Carotenoids and carotenogenesis in anoxygenic photosynthetic bacteria, in: H.A. Frank, A.J. Young, G. Britton, R.J. Cogdell (Eds.), The Photochemistry of Carotenoids: Advances in Photosynthesis, Kluwer Academic, Dordrecht, 1999, pp. 39-69.

[139] S. Takaichi, Distribution and biosynthesis of carotenoids. The Purple Phototrophic Bacteria, in: C.N. Hunter, F. Daldal, M.C. Thurnauer, J.T. Beaty (Eds.), Adv Photosynthesis Respiration2009, pp. 97-117.

[140] N.J. Fraser, H. Hashimoto, R.J. Cogdell, Carotenoids and bacterial photosynthesis: The story so far, Photosynth Res 70 (2001) 249-56.

[141] T.N. Toth, V. Chukhutsina, I. Domonkos, J. Knoppova, J. Komenda, M. Kis, Z. Lenart, G. Garab, L. Kovacs, Z. Gombos, H. van Amerongen, Carotenoids are essential for the assembly of cyanobacterial photosynthetic complexes, Biochim Biophys Acta 1847 (2015) 1153-65. 
[142] T. Zakar, H. Laczko-Dobos, T.N. Toth, Z. Gombos, Carotenoids Assist in Cyanobacterial Photosystem II Assembly and Function, Front Plant Sci 7 (2016) 295.

[143] D. Kirilovsky, C.A. Kerfeld, Cyanobacterial photoprotection by the orange carotenoid protein, Nat Plants 2 (2016) 16180.

[144] T.W. Goodwin, The nature and distribution of carotenoids in some blue-green algae, J Gen Microbiol 17 (1957) 467-73.

[145] S. Takaichi, Carotenoids in algae: distributions, biosyntheses and functions, Mar Drugs 9 (2011) 1101-18.

[146] S. Takaichi, M. Mochimaru, Carotenoids and carotenogenesis in cyanobacteria: unique ketocarotenoids and carotenoid glycosides, Cell Mol Life Sci 64 (2007) 2607-19.

[147] G. Sandmann, J. Mautz, J. Breitenbach, Control of light-dependent keto carotenoid biosynthesis in Nostoc 7120 by the transcription factor NtcA, Z Naturforsch C 71 (2016) 303311.

[148] K.J. Mulders, P.P. Lamers, D.E. Martens, R.H. Wijffels, Phototrophic pigment production with microalgae: biological constraints and opportunities, J Phycol 50 (2014) 229-42.

[149] M.F. Raposo, A.M. de Morais, R.M. de Morais, Carotenoids from Marine Microalgae: A Valuable Natural Source for the Prevention of Chronic Diseases, Mar Drugs 13 (2015) 5128-55.

[150] V. Henriquez, C. Escobar, J. Galarza, J. Gimpel, Carotenoids in Microalgae, Subcell Biochem 79 (2016) 219-37.

[151] K. Wichuk, S. Brynjolfsson, W. Fu, Biotechnological production of value-added carotenoids from microalgae: Emerging technology and prospects, Bioengineered 5 (2014) 204-8.

[152] A. Solovchenko, K. Neverov, Carotenogenic response in photosynthetic organisms: a colorful story, Photosynth Res 133 (2017) 31-47.

[153] A.E. Solovchenko, Physiological role of neutral lipid accumulation in eukaryotic microalgae under stresses, Russ. J. Plant Physiol. 59 (2012) 167-176.

[154] J.A. Del Campo, M. Garcia-Gonzalez, M.G. Guerrero, Outdoor cultivation of microalgae for carotenoid production: current state and perspectives, Appl Microbiol Biotechnol 74 (2007) 1163-74.

[155] A.C. Guedes, H.M. Amaro, F.X. Malcata, Microalgae as sources of carotenoids, Mar Drugs 9 (2011) 625-644.

[156] P.P. Lamers, M. Janssen, R.C. De Vos, R.J. Bino, R.H. Wijffels, Exploring and exploiting carotenoid accumulation in Dunaliella salina for cell-factory applications, Trends Biotechnol 26 (2008) 631-8.

[157] A. Oren, A hundred years of Dunaliella research: 1905-2005, Saline Systems 1 (2005) 2.

[158] M.M. Shah, Y. Liang, J.J. Cheng, M. Daroch, Astaxanthin-Producing Green Microalga Haematococcus pluvialis: From Single Cell to High Value Commercial Products, Front Plant Sci 7 (2016) 531.

[159] J. Avalos, V. Díaz-Sánchez, J. García-Martínez, M. Castrillo, M. Ruger-Herreros, M.C. Limón, Carotenoids, in: J.F. Martín, C. García-Estrada, S. Zeilinger (Eds.), Biosynthesis and Molecular Genetics of Fungal Secondary Metabolites, Springer2014, pp. 149-185.

[160] J. Avalos, S. Nordzieke, O. Parra, J. Pardo-Medina, M.C. Limón, Carotenoid production by filamentous fungi and yeasts, in: A.A. Sibirny (Ed.), Biotechnology of Yeasts and Filamentous Fungi2017. 
[161] A.F. Estrada, T. Brefort, C. Mengel, V. Diaz-Sanchez, A. Alder, S. Al-Babili, J. Avalos, Ustilago maydis accumulates beta-carotene at levels determined by a retinal-forming carotenoid oxygenase, Fungal Genet Biol 46 (2009) 803-13.

[162] M.E. Daub, G.A. Payne, The role of carotenoids in resistance of fungi to cercosporin, Phytopathology 79 (1989) 180-185.

[163] A. Prado-Cabrero, A.F. Estrada, S. Al-Babili, J. Avalos, Identification and biochemical characterization of a novel carotenoid oxygenase: elucidation of the cleavage step in the Fusarium carotenoid pathway, Mol Microbiol 64 (2007) 448-60.

[164] L. Saelices, L. Youssar, I. Holdermann, S. Al-Babili, J. Avalos, Identification of the gene responsible for torulene cleavage in the Neurospora carotenoid pathway, Mol Genet Genomics 278 (2007) 527-37.

[165] A.F. Estrada, L. Youssar, D. Scherzinger, S. Al-Babili, J. Avalos, The ylo-1 gene encodes an aldehyde dehydrogenase responsible for the last reaction in the Neurospora carotenoid pathway, Mol Microbiol 69 (2008) 1207-20.

[166] V. Diaz-Sanchez, A.F. Estrada, D. Trautmann, S. Al-Babili, J. Avalos, The gene carD encodes the aldehyde dehydrogenase responsible for neurosporaxanthin biosynthesis in Fusarium fujikuroi, FEBS J 278 (2011) 3164-76.

[167] A. Prado-Cabrero, D. Scherzinger, J. Avalos, S. Al-Babili, Retinal biosynthesis in fungi: characterization of the carotenoid oxygenase CarX from Fusarium fujikuroi, Eukaryot Cell 6 (2007) 650-7.

[168] J. Avalos, E. Cerdá-Olmedo, Fungal carotenoid production, in: D.K. Arora (Ed.), Handbook of Fungal Biotechnology, Marcel Dekker, Inc., New York, 2004, pp. 367-378.

[169] H. Pollmann, J. Breitenbach, G. Sandmann, Engineering of the carotenoid pathway in Xanthophyllomyces dendrorhous leading to the synthesis of zeaxanthin, Appl Microbiol Biotechnol 101 (2017) 103-111.

[170] N. Ledetzky, A. Osawa, K. Iki, H. Pollmann, S. Gassel, J. Breitenbach, K. Shindo, G. Sandmann, Multiple transformation with the crtYB gene of the limiting enzyme increased carotenoid synthesis and generated novel derivatives in Xanthophyllomyces dendrorhous, Arch Biochem Biophys 545 (2014) 141-7.

[171] J. Avalos, M.C. Limon, Biological roles of fungal carotenoids, Curr Genet 61 (2015) 309-24.

[172] M.M. Prado, A. Prado-Cabrero, R. Fernandez-Martin, J. Avalos, A gene of the opsin family in the carotenoid gene cluster of Fusarium fujikuroi, Curr Genet 46 (2004) 47-58.

[173] A.F. Estrada, J. Avalos, Regulation and targeted mutation of opsA, coding for the NOP-1 opsin orthologue in Fusarium fujikuroi, J Mol Biol 387 (2009) 59-73.

[174] R.P. Sutter, Mutations affecting sexual development in Phycomyces blakesleeanus, Proc Natl Acad Sci U S A 72 (1975) 127-30.

[175] A. Vershinin, Biological functions of carotenoids--diversity and evolution, Biofactors 10 (1999) 99-104.

[176] N.A. Moran, T. Jarvik, Lateral transfer of genes from fungi underlies carotenoid production in aphids, Science 328 (2010) 624-7.

[177] B. Altincicek, J.L. Kovacs, N.M. Gerardo, Horizontally transferred fungal carotenoid genes in the two-spotted spider mite Tetranychus urticae, Biol Lett 8 (2012) 253-7. 
[178] C. Cobbs, J. Heath, J.O. Stireman, 3rd, P. Abbot, Carotenoids in unexpected places: gall midges, lateral gene transfer, and carotenoid biosynthesis in animals, Mol Phylogenet Evol 68 (2013) 221-8.

[179] E. Novakova, N.A. Moran, Diversification of genes for carotenoid biosynthesis in aphids following an ancient transfer from a fungus, Mol Biol Evol 29 (2012) 313-23.

[180] C. Zhao, P.D. Nabity, Phylloxerids share ancestral carotenoid biosynthesis genes of fungal origin with aphids and adelgids, PLoS One 12 (2017) e0185484.

[181] M. Grbic, T. Van Leeuwen, R.M. Clark, S. Rombauts, P. Rouze, V. Grbic, E.J. Osborne, W. Dermauw, P.C. Ngoc, F. Ortego, P. Hernandez-Crespo, I. Diaz, M. Martinez, M. Navajas, E. Sucena, S. Magalhaes, L. Nagy, R.M. Pace, S. Djuranovic, G. Smagghe, M. Iga, O. Christiaens, J.A. Veenstra, J. Ewer, R.M. Villalobos, J.L. Hutter, S.D. Hudson, M. Velez, S.V. Yi, J. Zeng, A. Pires-daSilva, F. Roch, M. Cazaux, M. Navarro, V. Zhurov, G. Acevedo, A. Bjelica, J.A. Fawcett, E. Bonnet, C. Martens, G. Baele, L. Wissler, A. Sanchez-Rodriguez, L. Tirry, C. Blais, K. Demeestere, S.R. Henz, T.R. Gregory, J. Mathieu, L. Verdon, L. Farinelli, J. Schmutz, E. Lindquist, R. Feyereisen, Y. Van de Peer, The genome of Tetranychus urticae reveals herbivorous pest adaptations, Nature 479 (2011) 487-92.

[182] F.J. Schweigert, Metabolism of Carotenoids in Mammals, in: G. Britton, S. Liaaen-Jensen, H. Pfander (Eds.), Carotenoids Vol. 3: Biosynthesis and Metabolism, Birkhäuser Verlag, Basel, Switzerland, 1998, pp. 249-284.

[183] P. Nozière, B. Graulet, A. Lucas, B. Martin, P. Grolier, M. Doreau, Carotenoids for ruminants: From forages to dairy products, 2006, pp. 418-450.

[184] F. Khachik, C.J. Spangler, J.C. Smith, L.M. Canfield, A. Steck, H. Pfander, Identification, Quantification, and Relative Concentrations of Carotenoids and Their Metabolites in Human Milk and Serum, Analytical Chemistry 69 (1997) 1873-1881.

[185] E.J. Johnson, Role of lutein and zeaxanthin in visual and cognitive function throughout the lifespan, Nutrition Reviews 72 (2014) 605-612.

[186] A. Perry, H. Rasmussen, E.J. Johnson, Xanthophyll (lutein, zeaxanthin) content in fruits, vegetables and corn and egg products, Journal of Food Composition and Analysis 22 (2009) 915.

[187] A.M. LaFountain, R.O. Prum, H.A. Frank, Diversity, physiology, and evolution of avian plumage carotenoids and the role of carotenoid-protein interactions in plumage color appearance, Arch Biochem Biophys 572 (2015) 201-12.

[188] T. Maoka, Carotenoids in marine animals, 2011, pp. 278-293.

[189] D.E. Breithaupt, Modern application of xanthophylls in animal feeding - a review, 2007, pp. 501-506.

[190] M. Havaux, Carotenoid oxidation products as stress signals in plants, Plant J 79 (2014) 597606.

[191] X. Sui, P.D. Kiser, J. Lintig, K. Palczewski, Structural basis of carotenoid cleavage: from bacteria to mammals, Arch Biochem Biophys 539 (2013) 203-13.

[192] M.D. White, E. Flashman, Catalytic strategies of the non-heme iron dependent oxygenases and their roles in plant biology, Curr Opin Chem Biol 31 (2016) 126-35.

[193] L.A. Caceres, S. Lakshminarayan, K.K. Yeung, B.D. McGarvey, A. Hannoufa, M.W. Sumarah, X. Benitez, I.M. Scott, Repellent and Attractive Effects of alpha-, beta-, and Dihydro-betaIonone to Generalist and Specialist Herbivores, J Chem Ecol 42 (2016) 107-17. 
[194] A. Rodriguez, B. Alquezar, L. Pena, Fruit aromas in mature fleshy fruits as signals of readiness for predation and seed dispersal, New Phytol 197 (2013) 36-48.

[195] F.A. Macias, A. Lopez, R.M. Varela, A. Torres, J.M. Molinillo, Bioactive apocarotenoids annuionones F and G: structural revision of annuionones A, B and E, Phytochemistry 65 (2004) 3057-63.

[196] K. Akiyama, K. Matsuzaki, H. Hayashi, Plant sesquiterpenes induce hyphal branching in arbuscular mycorrhizal fungi, Nature 435 (2005) 824-7.

[197] M.H. Walter, D.S. Floss, D. Strack, Apocarotenoids: hormones, mycorrhizal metabolites and aroma volatiles, Planta 232 (2010) 1-17.

[198] R.P. McQuinn, J.J. Giovannoni, B.J. Pogson, More than meets the eye: from carotenoid biosynthesis, to new insights into apocarotenoid signaling, Curr Opin Plant Biol 27 (2015) 1729.

[199] S. Al-Babili, H.J. Bouwmeester, Strigolactones, a novel carotenoid-derived plant hormone, Annu Rev Plant Biol 66 (2015) 161-86.

[200] E. Adhikari, D.K. Lee, P. Giavalisco, L.E. Sieburth, Long-distance signaling in bypass1 mutants: bioassay development reveals the bps signal to be a metabolite, Mol Plant 6 (2013) 164-73.

[201] J.M. Van Norman, L.E. Sieburth, Dissecting the biosynthetic pathway for the bypass1 rootderived signal, Plant J 49 (2007) 619-28.

[202] A.O. Avendano-Vazquez, E. Cordoba, E. Llamas, C. San Roman, N. Nisar, S. De la Torre, M. Ramos-Vega, M.D. Gutierrez-Nava, C.I. Cazzonelli, B.J. Pogson, P. Leon, An Uncharacterized Apocarotenoid-Derived Signal Generated in zeta-Carotene Desaturase Mutants Regulates Leaf Development and the Expression of Chloroplast and Nuclear Genes in Arabidopsis, Plant Cell 26 (2014) 2524-2537.

[203] J.M. Van Norman, J. Zhang, C.I. Cazzonelli, B.J. Pogson, P.J. Harrison, T.D. Bugg, K.X. Chan, A.J. Thompson, P.N. Benfey, Periodic root branching in Arabidopsis requires synthesis of an uncharacterized carotenoid derivative, Proc Natl Acad Sci U S A 111 (2014) E1300-9.

[204] E. Christodoulou, N.P. Kadoglou, N. Kostomitsopoulos, G. Valsami, Saffron: a natural product with potential pharmaceutical applications, J Pharm Pharmacol 67 (2015) 1634-49.

[205] R. Rivera-Madrid, M. Aguilar-Espinosa, Y. Cardenas-Conejo, L.E. Garza-Caligaris, Carotenoid Derivates in Achiote (Bixa orellana) Seeds: Synthesis and Health Promoting Properties, Front Plant Sci 7 (2016) 1406.

[206] H.H. Li, R.L. Hao, S.S. Wu, P.C. Guo, C.J. Chen, L.P. Pan, H. Ni, Occurrence, function and potential medicinal applications of the phytohormone abscisic acid in animals and humans, Biochem Pharmacol 82 (2011) 701-12.

[207] M. Fridlender, Y. Kapulnik, H. Koltai, Plant derived substances with anti-cancer activity: from folklore to practice, Front Plant Sci 6 (2015) 799.

[208] M.P. Croglio, J.M. Haake, C.P. Ryan, V.S. Wang, J. Lapier, J.P. Schlarbaum, Y. Dayani, E. Artuso, C. Prandi, H. Koltai, K. Agama, Y. Pommier, Y. Chen, L. Tricoli, J.R. LaRocque, C. Albanese, R.I. Yarden, Analogs of the novel phytohormone, strigolactone, trigger apoptosis and synergize with PARP inhibitors by inducing DNA damage and inhibiting DNA repair, Oncotarget 7 (2016) 13984-4001.

[209] M. Ansari, S. Emami, beta-lonone and its analogs as promising anticancer agents, Eur J Med Chem 123 (2016) 141-154. 
[210] D.P. Kloer, G.E. Schulz, Structural and biological aspects of carotenoid cleavage, Cell Mol Life Sci 63 (2006) 2291-303.

[211] C. dela Sena, K.M. Riedl, S. Narayanasamy, R.W. Curley, Jr., S.J. Schwartz, E.H. Harrison, The human enzyme that converts dietary provitamin $A$ carotenoids to vitamin $A$ is a dioxygenase, $J$ Biol Chem 289 (2014) 13661-6.

[212] H. Schmidt, R. Kurtzer, W. Eisenreich, W. Schwab, The carotenase AtCCD1 from Arabidopsis thaliana is a dioxygenase, J Biol Chem 281 (2006) 9845-51.

[213] O. Ahrazem, L. Gomez-Gomez, M.J. Rodrigo, J. Avalos, M.C. Limon, Carotenoid Cleavage Oxygenases from Microbes and Photosynthetic Organisms: Features and Functions, Int J Mol Sci 17 (2016).

[214] S.H. Schwartz, B.C. Tan, D.A. Gage, J.A. Zeevaart, D.R. McCarty, Specific oxidative cleavage of carotenoids by VP14 of maize, Science 276 (1997) 1872-4.

[215] E. Nambara, A. Marion-Poll, Abscisic acid biosynthesis and catabolism, Annu Rev Plant Biol 56 (2005) 165-85.

[216] A. Alder, M. Jamil, M. Marzorati, M. Bruno, M. Vermathen, P. Bigler, S. Ghisla, H. Bouwmeester, P. Beyer, S. Al-Babili, The path from beta-carotene to carlactone, a strigolactone-like plant hormone, Science 335 (2012) 1348-51.

[217] H. Lin, R. Wang, Q. Qian, M. Yan, X. Meng, Z. Fu, C. Yan, B. Jiang, Z. Su, J. Li, Y. Wang, DWARF27, an iron-containing protein required for the biosynthesis of strigolactones, regulates rice tiller bud outgrowth, Plant Cell 21 (2009) 1512-25.

[218] M.T. Waters, P.B. Brewer, J.D. Bussell, S.M. Smith, C.A. Beveridge, The Arabidopsis ortholog of rice DWARF27 acts upstream of MAX1 in the control of plant development by strigolactones, Plant Physiol 159 (2012) 1073-85.

[219] S.H. Schwartz, X. Qin, J.A. Zeevaart, Characterization of a novel carotenoid cleavage dioxygenase from plants, J Biol Chem 276 (2001) 25208-11.

[220] A.J. Simkin, S.H. Schwartz, M. Auldridge, M.G. Taylor, H.J. Klee, The tomato carotenoid cleavage dioxygenase 1 genes contribute to the formation of the flavor volatiles beta-ionone, pseudoionone, and geranylacetone, Plant J 40 (2004) 882-92.

[221] R. Vallabhaneni, L.M. Bradbury, E.T. Wurtzel, The carotenoid dioxygenase gene family in maize, sorghum, and rice, Arch Biochem Biophys 504 (2010) 104-11.

[222] R. da Silva Messias, V. Galli, E.S.S.D. Dos Anjos, C.V. Rombaldi, Carotenoid biosynthetic and catabolic pathways: gene expression and carotenoid content in grains of maize landraces, Nutrients 6 (2014) 546-63.

[223] B.C. Tan, J.C. Guan, S. Ding, S. Wu, J.W. Saunders, K.E. Koch, D.R. McCarty, Structure and Origin of the White Cap Locus and Its Role in Evolution of Grain Color in Maize, Genetics 206 (2017) 135-150.

[224] D.S. Floss, M.H. Walter, Role of carotenoid cleavage dioxygenase 1 (CCD1) in apocarotenoid biogenesis revisited, Plant Signal Behav 4 (2009) 172-5.

[225] A. Ilg, P. Beyer, S. Al-Babili, Characterization of the rice carotenoid cleavage dioxygenase 1 reveals a novel route for geranial biosynthesis, FEBS J 276 (2009) 736-47.

[226] A. Ilg, M. Bruno, P. Beyer, S. Al-Babili, Tomato carotenoid cleavage dioxygenases 1A and 1B: Relaxed double bond specificity leads to a plenitude of dialdehydes, mono-apocarotenoids and isoprenoid volatiles, FEBS Open Bio 4 (2014) 584-93. 
[227] S. Frusciante, G. Diretto, M. Bruno, P. Ferrante, M. Pietrella, A. Prado-Cabrero, A. RubioMoraga, P. Beyer, L. Gomez-Gomez, S. Al-Babili, G. Giuliano, Novel carotenoid cleavage dioxygenase catalyzes the first dedicated step in saffron crocin biosynthesis, Proc Natl Acad Sci U S A 111 (2014) 12246-51.

[228] O. Ahrazem, A. Rubio-Moraga, J. Berman, T. Capell, P. Christou, C. Zhu, L. Gomez-Gomez, The carotenoid cleavage dioxygenase CCD2 catalysing the synthesis of crocetin in spring crocuses and saffron is a plastidial enzyme, New Phytol 209 (2016) 650-63.

[229] A. Rubio, J.L. Rambla, M. Santaella, M.D. Gomez, D. Orzaez, A. Granell, L. Gomez-Gomez, Cytosolic and plastoglobule-targeted carotenoid dioxygenases from Crocus sativus are both involved in beta-ionone release, J Biol Chem 283 (2008) 24816-25.

[230] F.C. Huang, P. Molnar, W. Schwab, Cloning and functional characterization of carotenoid cleavage dioxygenase 4 genes, J Exp Bot 60 (2009) 3011-22.

[231] M. Bruno, P. Beyer, S. Al-Babili, The potato carotenoid cleavage dioxygenase 4 catalyzes a single cleavage of beta-ionone ring-containing carotenes and non-epoxidated xanthophylls, Arch Biochem Biophys 572 (2015) 126-33.

[232] M.J. Rodrigo, B. Alquezar, E. Alos, V. Medina, L. Carmona, M. Bruno, S. Al-Babili, L. Zacarias, A novel carotenoid cleavage activity involved in the biosynthesis of Citrus fruit-specific apocarotenoid pigments, J Exp Bot 64 (2013) 4461-78.

[233] M. Bruno, J. Koschmieder, F. Wuest, P. Schaub, M. Fehling-Kaschek, J. Timmer, P. Beyer, S. Al-Babili, Enzymatic study on AtCCD4 and AtCCD7 and their potential to form acyclic regulatory metabolites, J Exp Bot 67 (2016) 5993-6005.

[234] G. Ma, L. Zhang, A. Matsuta, K. Matsutani, K. Yamawaki, M. Yahata, A. Wahyudi, R. Motohashi, M. Kato, Enzymatic formation of beta-citraurin from beta-cryptoxanthin and Zeaxanthin by carotenoid cleavage dioxygenase 4 in the flavedo of citrus fruit, Plant Physiol 163 (2013) 682-95.

[235] O. Ahrazem, G. Diretto, J. Argandona, A. Rubio-Moraga, J.M. Julve, D. Orzaez, A. Granell, L. Gomez-Gomez, Evolutionarily distinct carotenoid cleavage dioxygenases are responsible for crocetin production in Buddleja davidii, J Exp Bot 68 (2017) 4663-4677.

[236] S. Gonzalez-Jorge, S.H. Ha, M. Magallanes-Lundback, L.U. Gilliland, A. Zhou, A.E. Lipka, Y.N. Nguyen, R. Angelovici, H. Lin, J. Cepela, H. Little, C.R. Buell, M.A. Gore, D. Dellapenna, Carotenoid cleavage dioxygenase 4 is a negative regulator of beta-carotene content in Arabidopsis seeds, Plant Cell 25 (2013) 4812-26.

[237] R. Campbell, L.J. Ducreux, W.L. Morris, J.A. Morris, J.C. Suttle, G. Ramsay, G.J. Bryan, P.E. Hedley, M.A. Taylor, The metabolic and developmental roles of carotenoid cleavage dioxygenase4 from potato, Plant Physiol 154 (2010) 656-64.

[238] G. Farre, G. Sanahuja, S. Naqvi, C. Bai, T. Capell, C. Zhu, P. Christou, Travel advice on the road to carotenoids in plants, Plant Science 179 (2010) 28-48.

[239] G. Giuliano, Provitamin A biofortification of crop plants: a gold rush with many miners, Curr Opin Biotechnol 44 (2017) 169-180.

[240] V. Mann, M. Harker, I. Pecker, J. Hirschberg, Metabolic engineering of astaxanthin production in tobacco flowers, Nat Biotechnol 18 (2000) 888-92.

[241] C. Zhu, T. Gerjets, G. Sandmann, Nicotiana glauca engineered for the production of ketocarotenoids in flowers and leaves by expressing the cyanobacterial crtO ketolase gene, Transgenic Res 16 (2007) 813-21. 
[242] T. Gerjets, M. Sandmann, C. Zhu, G. Sandmann, Metabolic engineering of ketocarotenoid biosynthesis in leaves and flowers of tobacco species, Biotechnol J 2 (2007) 1263-9.

[243] T. Hasunuma, S. Miyazawa, S. Yoshimura, Y. Shinzaki, K. Tomizawa, K. Shindo, S.K. Choi, N. Misawa, C. Miyake, Biosynthesis of astaxanthin in tobacco leaves by transplastomic engineering, Plant J 55 (2008) 857-68.

[244] Y. Lu, S. Stegemann, S. Agrawal, D. Karcher, S. Ruf, R. Bock, Horizontal Transfer of a Synthetic Metabolic Pathway between Plant Species, Curr Biol 27 (2017) 3034-3041 e3.

[245] C.L. Mortimer, N. Misawa, L. Perez-Fons, F.P. Robertson, H. Harada, P.M. Bramley, P.D. Fraser, The Formation and Sequestration of Nonendogenous Ketocarotenoids in Transgenic Nicotiana glauca, Plant Physiol 173 (2017) 1617-1635.

[246] C.L. Mortimer, N. Misawa, L. Ducreux, R. Campbell, P.M. Bramley, M. Taylor, P.D. Fraser, Product stability and sequestration mechanisms in Solanum tuberosum engineered to biosynthesize high value ketocarotenoids, Plant Biotechnol J 14 (2016) 140-52.

[247] H. Harada, T. Maoka, A. Osawa, J. Hattan, H. Kanamoto, K. Shindo, T. Otomatsu, N. Misawa, Construction of transplastomic lettuce (Lactuca sativa) dominantly producing astaxanthin fatty acid esters and detailed chemical analysis of generated carotenoids, Transgenic Res 23 (2014) 303-15.

[248] K. Simpson, L.F. Quiroz, M. Rodriguez-Concepcion, C.R. Stange, Differential Contribution of the First Two Enzymes of the MEP Pathway to the Supply of Metabolic Precursors for Carotenoid and Chlorophyll Biosynthesis in Carrot (Daucus carota), Front Plant Sci 7 (2016) 1344.

[249] D. Maass, J. Arango, F. Wust, P. Beyer, R. Welsch, Carotenoid crystal formation in Arabidopsis and carrot roots caused by increased phytoene synthase protein levels, PLoS One 4 (2009) e6373.

[250] J.C. Moreno, L. Pizarro, P. Fuentes, M. Handford, V. Cifuentes, C. Stange, Levels of lycopene beta-cyclase 1 modulate carotenoid gene expression and accumulation in Daucus carota, PLoS One 8 (2013) e58144.

[251] J.C. Moreno, A. Cerda, K. Simpson, I. Lopez-Diaz, E. Carrera, M. Handford, C. Stange, Increased Nicotiana tabacum fitness through positive regulation of carotenoid, gibberellin and chlorophyll pathways promoted by Daucus carota lycopene beta-cyclase (Dclcyb1) expression, J Exp Bot 67 (2016) 2325-38.

[252] J. Jayaraj, R. Devlin, Z. Punja, Metabolic engineering of novel ketocarotenoid production in carrot plants, Transgenic Res 17 (2008) 489-501.

[253] R.G. Fray, A. Wallace, P.D. Fraser, D. Valero, P. Hedden, P.M. Bramley, D. Grierson, Constitutive expression of a fruit phytoene synthase gene in transgenic tomatoes causes dwarfism by redirecting metabolites from the gibberellin pathway, Plant J 8 (1995) 693-701.

[254] L.M. Lois, M. Rodríguez-Concepción, F. Gallego, N. Campos, A. Boronat, Carotenoid biosynthesis during tomato fruit development: regulatory role of 1-deoxy-D-xylulose 5phosphate synthase., Plant J 22 (2000) 503-513.

[255] E.M.A. Enfissi, P.D. Fraser, L.M. Lois, A. Boronat, W. Schuch, P.M. Bramley, Metabolic engineering of the mevalonate and non-mevalonate isopentenyl diphosphate-forming pathways for the production of health-promoting isoprenoids in tomato, Plant Biotech $\mathrm{J} 3$ (2005) 17-27. 
[256] S. Romer, P.D. Fraser, J.W. Kiano, C.A. Shipton, N. Misawa, W. Schuch, P.M. Bramley, Elevation of the provitamin A content of transgenic tomato plants, Nat Biotechnol 18 (2000) 666-9.

[257] E.M. Enfissi, M. Nogueira, P.M. Bramley, P.D. Fraser, The regulation of carotenoid formation in tomato fruit, Plant J 89 (2017) 774-788.

[258] C. D’Ambrosio, G. Giorio, I. Marino, A. Merendino, A. Petrozza, L. Salfi, A.L. Stigliani, F. Cellini, Virtually complete conversion of lycopene into $\beta$-carotene in fruits of tomato plants transformed with the tomato lycopene $\beta$-cyclase (tlcy-b) cDNA, Plant Science 166 (2004) 207214.

[259] L. Ralley, W. Schuch, P.D. Fraser, P.M. Bramley, Genetic modification of tomato with the tobacco lycopene beta-cyclase gene produces high beta-carotene and lycopene fruit, $Z$ Naturforsch C 71 (2016) 295-301.

[260] L. Ralley, E.M. Enfissi, N. Misawa, W. Schuch, P.M. Bramley, P.D. Fraser, Metabolic engineering of ketocarotenoid formation in higher plants, Plant J 39 (2004) 477-86.

[261] J.C. Huang, Y.J. Zhong, J. Liu, G. Sandmann, F. Chen, Metabolic engineering of tomato for high-yield production of astaxanthin, Metab Eng 17 (2013) 59-67.

[262] M. Nogueira, E.M.A. Enfissi, M.E. Martinez Valenzuela, G.N. Menard, R.L. Driller, P.J. Eastmond, W. Schuch, G. Sandmann, P.D. Fraser, Engineering of tomato for the sustainable production of ketocarotenoids and its evaluation in aquaculture feed, Proc Natl Acad Sci U S A 114 (2017) 10876-10881.

[263] E. Pons, B. Alquezar, A. Rodriguez, P. Martorell, S. Genoves, D. Ramon, M.J. Rodrigo, L. Zacarias, L. Pena, Metabolic engineering of beta-carotene in orange fruit increases its in vivo antioxidant properties, Plant Biotechnol J 12 (2014) 17-27.

[264] L.J.M. Ducreux, W.L. Morris, P.E. Hedley, T. Shepherd, H.V. Davies, S. Millam, M.A. Taylor, Metabolic engineering of high carotenoid potato tubers containing enhanced levels of betacarotene and lutein, J Exp Bot 56 (2005) 81-89.

[265] W.L. Morris, L.J. Ducreux, P. Hedden, S. Millam, M.A. Taylor, Overexpression of a bacterial 1-deoxy-D-xylulose 5-phosphate synthase gene in potato tubers perturbs the isoprenoid metabolic network: implications for the control of the tuber life cycle., J Exp Bot 57 (2006) 3007-3018.

[266] G. Diretto, S. Al-Babili, R. Tavazza, V. Papacchioli, P. Beyer, G. Giuliano, Metabolic engineering of potato carotenoid content through tuber-specific overexpression of a bacterial mini-pathway, PLoS One 2 (2007) e350.

[267] G. Diretto, R. Tavazza, R. Welsch, D. Pizzichini, F. Mourgues, V. Papacchioli, P. Beyer, G. Giuliano, Metabolic engineering of potato tuber carotenoids through tuber-specific silencing of lycopene epsilon cyclase, BMC Plant Biol 6 (2006) 13.

[268] G. Diretto, R. Welsch, R. Tavazza, F. Mourgues, D. Pizzichini, P. Beyer, G. Giuliano, Silencing of beta-carotene hydroxylase increases total carotenoid and beta-carotene levels in potato tubers, BMC Plant Biol 7 (2007) 11.

[269] S. Romer, J. Lubeck, F. Kauder, S. Steiger, C. Adomat, G. Sandmann, Genetic engineering of a zeaxanthin-rich potato by antisense inactivation and co-suppression of carotenoid epoxidation, Metab Eng 4 (2002) 263-72.

[270] T. Gerjets, G. Sandmann, Ketocarotenoid formation in transgenic potato, J Exp Bot 57 (2006) 3639-45. 
[271] W.L. Morris, L.J. Ducreux, P.D. Fraser, S. Millam, M.A. Taylor, Engineering ketocarotenoid biosynthesis in potato tubers, Metab Eng 8 (2006) 253-63.

[272] L. Li, Y. Yang, Q. Xu, K. Owsiany, R. Welsch, C. Chitchumroonchokchai, S. Lu, J. Van Eck, X.X. Deng, M. Failla, T.W. Thannhauser, The Or gene enhances carotenoid accumulation and stability during post-harvest storage of potato tubers, Mol Plant 5 (2012) 339-52.

[273] R. Campbell, W.L. Morris, C.L. Mortimer, N. Misawa, L.J. Ducreux, J.A. Morris, P.E. Hedley, P.D. Fraser, M.A. Taylor, Optimising ketocarotenoid production in potato tubers: effect of genetic background, transgene combinations and environment, Plant Sci 234 (2015) 27-37.

[274] S.C. Park, S.H. Kim, S. Park, H.U. Lee, J.S. Lee, W.S. Park, M.J. Ahn, Y.H. Kim, J.C. Jeong, H.S. Lee, S.S. Kwak, Enhanced accumulation of carotenoids in sweetpotato plants overexpressing IbOr-Ins gene in purple-fleshed sweetpotato cultivar, Plant Physiol Biochem 86 (2015) 82-90.

[275] C.K. Shewmaker, J.A. Sheehy, M. Daley, S. Colburn, D.Y. Ke, Seed-specific overexpression of phytoene synthase: increase in carotenoids and other metabolic effects, Plant J 20 (1999) 401412.

[276] M.P. Ravanello, D. Ke, J. Alvarez, B. Huang, C.K. Shewmaker, Coordinate expression of multiple bacterial carotenoid genes in canola leading to altered carotenoid production, Metab Eng 5 (2003) 255-263.

[277] M. Fujisawa, E. Takita, H. Harada, N. Sakurai, H. Suzuki, K. Ohyama, D. Shibata, N. Misawa, Pathway engineering of Brassica napus seeds using multiple key enzyme genes involved in ketocarotenoid formation, J Exp Bot 60 (2009) 1319-32.

[278] B. Yu, D.J. Lydiate, L.W. Young, U.A. Schafer, A. Hannoufa, Enhancing the carotenoid content of Brassica napus seeds by downregulating lycopene epsilon cyclase, Transgenic Res 17 (2008) 573-85.

[279] M.J. Kim, J.K. Kim, H.J. Kim, J.H. Pak, J.H. Lee, D.H. Kim, H.K. Choi, H.W. Jung, J.D. Lee, Y.S. Chung, S.H. Ha, Genetic modification of the soybean to enhance the beta-carotene content through seed-specific expression, PLoS One 7 (2012) e48287.

[280] M.A. Schmidt, W.A. Parrott, D.F. Hildebrand, R.H. Berg, A. Cooksey, K. Pendarvis, Y. He, F. McCarthy, E.M. Herman, Transgenic soya bean seeds accumulating beta-carotene exhibit the collateral enhancements of oleate and protein content traits, Plant Biotechnol J 13 (2015) 590600.

[281] E.C. Pierce, P.R. LaFayette, M.A. Ortega, B.L. Joyce, D.A. Kopsell, W.A. Parrott, Ketocarotenoid Production in Soybean Seeds through Metabolic Engineering, PLoS One 10 (2015) e0138196.

[282] X. Ye, S. Al-Babili, A. Kloti, J. Zhang, P. Lucca, P. Beyer, I. Potrykus, Engineering the provitamin A (beta-carotene) biosynthetic pathway into (carotenoid-free) rice endosperm, Science 287 (2000) 303-305.

[283] J.A. Paine, C.A. Shipton, S. Chaggar, R.M. Howells, M.J. Kennedy, G. Vernon, S.Y. Wright, E. Hinchliffe, J.L. Adams, A.L. Silverstone, R. Drake, Improving the nutritional value of Golden Rice through increased pro-vitamin A content, Nat Biotechnol 23 (2005) 482-487.

[284] T.N. Pham, T.L. Dong, V.H. Tran, T.C.H. Tran, Effect of storage conditions on total carotenoid content in golden rice grains, Omonrice 14 (2006) 18-27.

[285] D. Gayen, N. Ali, S.N. Sarkar, S.K. Datta, K. Datta, Down-regulation of lipoxygenase gene reduces degradation of carotenoids of golden rice during storage, Planta 242 (2015) 353-63. 
[286] C. Bai, J. Berman, G. Farre, T. Capell, G. Sandmann, P. Christou, C. Zhu, Reconstruction of the astaxanthin biosynthesis pathway in rice endosperm reveals a metabolic bottleneck at the level of endogenous beta-carotene hydroxylase activity, Transgenic Res 26 (2017) 13-23.

[287] M. Aluru, Y. Xu, R. Guo, Z. Wang, S. Li, W. White, K. Wang, S. Rodermel, Generation of transgenic maize with enhanced provitamin A content, J Exp Bot 59 (2008) 3551-62.

[288] C. Zhu, S. Naqvi, J. Breitenbach, G. Sandmann, P. Christou, T. Capell, Combinatorial genetic transformation generates a library of metabolic phenotypes for the carotenoid pathway in maize, Proc Natl Acad Sci U S A 105 (2008) 18232-7.

[289] S. Naqvi, C. Zhu, G. Farre, G. Sandmann, T. Capell, P. Christou, Synergistic metabolism in hybrid corn indicates bottlenecks in the carotenoid pathway and leads to the accumulation of extraordinary levels of the nutritionally important carotenoid zeaxanthin, Plant Biotechnol $\mathrm{J} 9$ (2011) 384-93.

[290] J. Berman, U. Zorrilla-Lopez, G. Sandmann, T. Capell, P. Christou, C. Zhu, The Silencing of Carotenoid beta-Hydroxylases by RNA Interference in Different Maize Genetic Backgrounds Increases the beta-Carotene Content of the Endosperm, Int J Mol Sci 18 (2017).

[291] G. Farre, L. Perez-Fons, M. Decourcelle, J. Breitenbach, S. Hem, C. Zhu, T. Capell, P. Christou, P.D. Fraser, G. Sandmann, Metabolic engineering of astaxanthin biosynthesis in maize endosperm and characterization of a prototype high oil hybrid, Transgenic Res 25 (2016) 47789.

[292] P. Che, Z.Y. Zhao, K. Glassman, D. Dolde, T.X. Hu, T.J. Jones, S. Obukosia, F. Wambugu, M.C. Albertsen, Elevated vitamin $\mathrm{E}$ content improves all-trans beta-carotene accumulation and stability in biofortified sorghum, Proc Natl Acad Sci U S A 113 (2016) 11040-5.

[293] L. Cong, C. Wang, L. Chen, H. Liu, G. Yang, G. He, Expression of phytoene synthase1 and carotene desaturase crtl genes result in an increase in the total carotenoids content in transgenic elite wheat (Triticum aestivum L.), J Agric Food Chem 57 (2009) 8652-60.

[294] C. Wang, J. Zeng, Y. Li, W. Hu, L. Chen, Y. Miao, P. Deng, C. Yuan, C. Ma, X. Chen, M. Zang, Q. Wang, K. Li, J. Chang, Y. Wang, G. Yang, G. He, Enrichment of provitamin A content in wheat (Triticum aestivum L.) by introduction of the bacterial carotenoid biosynthetic genes CrtB and Crtl, J Exp Bot 65 (2014) 2545-56.

[295] J. Zeng, X. Wang, Y. Miao, C. Wang, M. Zang, X. Chen, M. Li, X. Li, Q. Wang, K. Li, J. Chang, Y. Wang, G. Yang, G. He, Metabolic Engineering of Wheat Provitamin A by Simultaneously Overexpressing CrtB and Silencing Carotenoid Hydroxylase (TaHYD), J Agric Food Chem 63 (2015) 9083-92.

[296] T. Grune, G. Lietz, A. Palou, A.C. Ross, W. Stahl, G. Tang, D. Thurnham, S.A. Yin, H.K. Biesalski, Beta-carotene is an important vitamin A source for humans, J Nutr 140 (2010) 2268S-2285S.

[297] G. Maiani, M.J. Caston, G. Catasta, E. Toti, I.G. Cambrodon, A. Bysted, F. Granado-Lorencio, B. Olmedilla-Alonso, P. Knuthsen, M. Valoti, V. Bohm, E. Mayer-Miebach, D. Behsnilian, U. Schlemmer, Carotenoids: actual knowledge on food sources, intakes, stability and bioavailability and their protective role in humans, Mol Nutr Food Res 53 Suppl 2 (2009) S194218.

[298] A.N. Howard, D.I. Thurnham, Lutein and atherosclerosis: Belfast versus Toulouse revisited, Med Hypotheses 98 (2017) 63-68.

[299] X.D. Wang, Lycopene metabolism and its biological significance, Am J Clin Nutr 96 (2012) 1214S-22S. 
[300] M. Yamaguchi, Role of carotenoid beta-cryptoxanthin in bone homeostasis, J Biomed Sci 19 (2012) 36.

[301] D.A. Cooper, Carotenoids in health and disease: recent scientific evaluations, research recommendations and the consumer, J Nutr 134 (2004) 221S-224S.

[302] E. Fernández-García, I. Carvajal-Lérida, M. Jarén-Galán, J. Garrido-Fernández, A. PérezGálvez, D. Hornero-Méndez, Carotenoids bioavailability from foods: From plant pigments to efficient biological activities, Food Research International 46 (2012) 438-450.

[303] J.T. Landrum, R.A. Bone, Lutein, zeaxanthin, and the macular pigment, Arch Biochem Biophys 385 (2001) 28-40.

[304] M.L. Bonet, J.A. Canas, J. Ribot, A. Palou, Carotenoids and their conversion products in the control of adipocyte function, adiposity and obesity, Arch Biochem Biophys 572 (2015) 112-25.

[305] M. Hamer, Y. Chida, Intake of fruit, vegetables, and antioxidants and risk of type 2 diabetes: systematic review and meta-analysis, J Hypertens 25 (2007) 2361-9.

[306] E.T. Leermakers, S.K. Darweesh, C.P. Baena, E.M. Moreira, D. Melo van Lent, M.J. Tielemans, T. Muka, A. Vitezova, R. Chowdhury, W.M. Bramer, J.C. Kiefte-de Jong, J.F. Felix, O.H. Franco, The effects of lutein on cardiometabolic health across the life course: a systematic review and meta-analysis, Am J Clin Nutr 103 (2016) 481-94.

[307] A. Milani, M. Basirnejad, S. Shahbazi, A. Bolhassani, Carotenoids: biochemistry, pharmacology and treatment, Br J Pharmacol 174 (2017) 1290-1324.

[308] G. Britton, F. Khachik, Carotenoids in Food, in: G. Britton, S. Liaaen-Jensen, H. Pfander (Eds.), Carotenoids Vol. 5: Nutrition and Health, Birkhauser, Basel, Switzerland, 2009, pp. 4566.

[309] C.A. Lindbergh, L.M. Renzi-Hammond, B.R. Hammond, D.P. Terry, C.M. Mewborn, A.N. Puente, L.S. Miller, Lutein and Zeaxanthin Influence Brain Function in Older Adults: A Randomized Controlled Trial, J Int Neuropsychol Soc 24 (2018) 77-90.

[310] B.R. Hammond, Dietary Carotenoids and the Nervous System, Foods 4 (2015) 698-701.

[311] E.J. Johnson, A possible role for lutein and zeaxanthin in cognitive function in the elderly, Am J Clin Nutr 96 (2012) 1161S-5S.

[312] A.J. Meléndez-Martínez, P. Mapelli-Brahm, C.M. Stinco, The colourless carotenoids phytoene and phytofluene: From dietary sources to their usefulness for the functional foods and nutricosmetics industries, J. Food Compos. Anal. 67 (2018) 91-103.

[313] G. Bjelakovic, D. Nikolova, L.L. Gluud, R.G. Simonetti, C. Gluud, Antioxidant supplements for prevention of mortality in healthy participants and patients with various diseases, Cochrane Database Syst Rev (2012) CD007176.

[314] TATBCCPG, The alpha-tocopherol, beta-carotene lung cancer prevention study: design, methods, participant characteristics, and compliance. The ATBC Cancer Prevention Study Group, Ann Epidemiol 4 (1994) 1-10.

[315] G.S. Omenn, G.E. Goodman, M.D. Thornquist, J. Balmes, M.R. Cullen, A. Glass, J.P. Keogh, F.L. Meyskens, B. Valanis, J.H. Williams, S. Barnhart, S. Hammar, Effects of a combination of beta carotene and vitamin A on lung cancer and cardiovascular disease, N Engl J Med 334 (1996) 1150-5.

[316] D. Albanes, O.P. Heinonen, P.R. Taylor, J. Virtamo, B.K. Edwards, M. Rautalahti, A.M. Hartman, J. Palmgren, L.S. Freedman, J. Haapakoski, M.J. Barrett, P. Pietinen, N. Malila, E. Tala, K. Liippo, E.R. Salomaa, J.A. Tangrea, L. Teppo, F.B. Askin, E. Taskinen, Y. Erozan, P. Greenwald, 
J.K. Huttunen, Alpha-Tocopherol and beta-carotene supplements and lung cancer incidence in the alpha-tocopherol, beta-carotene cancer prevention study: effects of base-line characteristics and study compliance, J Natl Cancer Inst 88 (1996) 1560-70.

[317] E.R. Monsen, Dietary reference intakes for the antioxidant nutrients: vitamin C, vitamin E, selenium, and carotenoids, J Am Diet Assoc 100 (2000) 637-40.

[318] C.H. Hennekens, J.E. Buring, J.E. Manson, M. Stampfer, B. Rosner, N.R. Cook, C. Belanger, F. LaMotte, J.M. Gaziano, P.M. Ridker, W. Willett, R. Peto, Lack of effect of long-term supplementation with beta carotene on the incidence of malignant neoplasms and cardiovascular disease, N Engl J Med 334 (1996) 1145-9.

[319] W.J. Blot, J.Y. Li, P.R. Taylor, W. Guo, S.M. Dawsey, B. Li, The Linxian trials: mortality rates by vitamin-mineral intervention group, Am J Clin Nutr 62 (1995) 1424S-1426S.

[320] G. Tang, Bioconversion of dietary provitamin A carotenoids to vitamin A in humans, Am J Clin Nutr 91 (2010) 1468S-1473S.

[321] M.L. Bonet, J. Ribot, A. Palou, Lipid metabolism in mammalian tissues and its control by retinoic acid, Biochim Biophys Acta 1821 (2012) 177-89.

[322] P.J. Brun, K.J. Yang, S.A. Lee, J.J. Yuen, W.S. Blaner, Retinoids: Potent regulators of metabolism, Biofactors 39 (2013) 151-63.

[323] Y. Liu, H. Chen, D. Mu, J. Fan, J. Song, Y. Zhong, D. Li, M. Xia, Circulating Retinoic Acid Levels and the Development of Metabolic Syndrome, J Clin Endocrinol Metab 101 (2016) 1686-92.

[324] F. Serra, M.L. Bonet, P. Puigserver, J. Oliver, A. Palou, Stimulation of uncoupling protein 1 expression in brown adipocytes by naturally occurring carotenoids, Int J Obes Relat Metab Disord 23 (1999 ) 650-655.

[325] J. Amengual, E. Gouranton, Y.G. van Helden, S. Hessel, J. Ribot, E. Kramer, B. Kiec-Wilk, U. Razny, G. Lietz, A. Wyss, A. Dembinska-Kiec, A. Palou, J. Keijer, J.F. Landrier, M.L. Bonet, J. von Lintig, Beta-carotene reduces body adiposity of mice via BCMO1, PLoS One 6 (2011) e20644.

[326] S. Carpentier, M. Knaus, M. Suh, Associations between lutein, zeaxanthin, and age-related macular degeneration: an overview, Crit Rev Food Sci Nutr 49 (2009) 313-26.

[327] S. Sabour-Pickett, J.M. Nolan, J. Loughman, S. Beatty, A review of the evidence germane to the putative protective role of the macular carotenoids for age-related macular degeneration, Mol Nutr Food Res 56 (2012) 270-86.

[328] P.S. Bernstein, B. Li, P.P. Vachali, A. Gorusupudi, R. Shyam, B.S. Henriksen, J.M. Nolan, Lutein, zeaxanthin, and meso-zeaxanthin: The basic and clinical science underlying carotenoidbased nutritional interventions against ocular disease, Prog Retin Eye Res 50 (2016) 34-66.

[329] J.M. Nolan, K. Meagher, S. Kashani, S. Beatty, What is meso-zeaxanthin, and where does it come from?, Eye (Lond) 27 (2013) 899-905.

[330] N.I. Krinsky, J.T. Landrum, R.A. Bone, Biologic mechanisms of the protective role of lutein and zeaxanthin in the eye, Annu Rev Nutr 23 (2003) 171-201.

[331] E.Y. Chew, T.E. Clemons, J.P. Sangiovanni, R.P. Danis, F.L. Ferris, 3rd, M.J. Elman, A.N. Antoszyk, A.J. Ruby, D. Orth, S.B. Bressler, G.E. Fish, G.B. Hubbard, M.L. Klein, S.R. Chandra, B.A. Blodi, A. Domalpally, T. Friberg, W.T. Wong, P.J. Rosenfeld, E. Agron, C.A. Toth, P.S. Bernstein, R.D. Sperduto, Secondary analyses of the effects of lutein/zeaxanthin on agerelated macular degeneration progression: AREDS2 report No. 3, JAMA Ophthalmol 132 (2014) 142-9. 
[332] J. Mares, Lutein and Zeaxanthin Isomers in Eye Health and Disease, Annu Rev Nutr 36 (2016) 571-602.

[333] A.V. Rao, L.G. Rao, Carotenoids and human health, Pharmacol Res 55 (2007) 207-16.

[334] A.J. Melendez-Martinez, C.M. Stinco, C. Liu, X.D. Wang, A simple HPLC method for the comprehensive analysis of cis/trans (Z/E) geometrical isomers of carotenoids for nutritional studies, Food Chem 138 (2013) 1341-50.

[335] M.G. Dias, B. Olmedilla-Alonso, D. Hornero-Méndez, A.Z. Mercadante, C. Osorio, L. VargasMurga, A.J. Meléndez-Martínez, Tabla de contenido en carotenoides de alimentos iberoamericanos, in: A.J. Meléndez-Martínez (Ed.), Carotenoides en Agroalimentación y Salud, Terracota, , pp.. Ciudad de México, México, 2017, pp. 354-429.

[336] D. Hornero-Mendez, J. Costa-Garcia, M.I. Minguez-Mosquera, Characterization of carotenoid high-producing Capsicum annuum cultivars selected for paprika production, J Agric Food Chem 50 (2002) 5711-6.

[337] M.J. Rodrigo, J.F. Marcos, L. Zacarias, Biochemical and molecular analysis of carotenoid biosynthesis in flavedo of orange (Citrus sinensis L.) during fruit development and maturation, J Agric Food Chem 52 (2004) 6724-31.

[338] D.E. Breithaupt, A. Bamedi, Carotenoid esters in vegetables and fruits: a screening with emphasis on beta-cryptoxanthin esters, J Agric Food Chem 49 (2001) 2064-70.

[339] B.J. Burri, Beta-cryptoxanthin as a source of vitamin A, J Sci Food Agric 95 (2015) 1786-94.

[340] M.G. Sajilata, R.S. Singhal, M.Y. Kamat, The carotenoid pigment zeaxanthin-A review, Comp Rev Food Sci Food Safety 7 (2008) 29-49.

[341] M.M. Calvo, Lutein: a valuable ingredient of fruit and vegetables, Crit Rev Food Sci Nutr 45 (2005) 671-96.

[342] F. Granado, B. Olmedilla, I. Blanco, E. Rojas Hidalgo, Carotenoid composition in raw and cooked Spanish vegetables., J. Agric. Food Chem. 40 (1992) 21352140.

[343] J. Shi, M. Le Maguer, Lycopene in tomatoes: chemical and physical properties affected by food processing, Crit Rev Biotechnol 20 (2000) 293-334.

[344] R. Delgado-Pelayo, L. Gallardo-Guerrero, D. Hornero-Mendez, Carotenoid composition of strawberry tree (Arbutus unedo L.) fruits, Food Chem 199 (2016) 165-75.

[345] P. Morales, I.C.F.R. Ferreira, A.M. Carvalho, V. Fernández-Ruiz, M.C. Sánchez-Mata, M. Cámara, R. Morales, J. Tardío, Wild edible fruits as a potential source of phytochemicals with capacity to inhibit lipid peroxidation, Eur. J. Lipid Sci. Technol. 115 (2013) 176-185.

[346] V.V. de Rosso, A.Z. Mercadante, Identification and quantification of carotenoids, by HPLCPDA-MS/MS, from Amazonian fruits, J Agric Food Chem 55 (2007) 5062-72.

[347] M.C. Sánchez-Mata, R.D. Cabrera Loera, P. Morales, V. Fernández-Ruiz, M. Cámara, C. Díez Marqués, M. Pardo-de-Santayana, J. Tardío, Wild vegetables of the Mediterranean area as valuable sources of bioactive compounds, Genetic Resources and Crop Evolution 59 (2012) 431-443.

[348] E. Murillo, A.J. Meléndez-Martínez, F. Portugal, Screening of vegetables and fruits from Panama for rich sources of lutein and zeaxanthin, Food Chemistry 122 (2010) 167-172.

[349] P.R. Shewry, S. Hey, Do "ancient" wheat species differ from modern bread wheat in their contents of bioactive components?, J. Cereal Sci. 65 (2015) 236-243. 
[350] S.G. Atienza, J. Ballesteros, A. Martin, D. Hornero-Mendez, Genetic variability of carotenoid concentration and degree of esterification among tritordeum (xTritordeum Ascherson et Graebner) and durum wheat accessions, J Agric Food Chem 55 (2007) 4244-51.

[351] C.R. Brown, Breeding for phytonutrient enhancement of potato, American J. Potato Res. 85 (2008) 298-307.

[352] G. Britton, Overview on carotenoid biosynthesis, in: G. Britton, S. Liaaen-Jensen, H. Pfander (Eds.), Carotenoids, vol. 3: Biosynthesis, Birkhäuser Verlag, Basel, Switzerland, 1998, pp. 13147.

[353] B. Camara, J. Brangeon, Carotenoid metabolism during chloroplast to chromoplast transformation in Capsicum annuum fruit, Planta 151 (1981) 359-64.

[354] D. Hornero-Mendez, M.I. Minguez-Mosquera, Xanthophyll esterification accompanying carotenoid overaccumulation in chromoplast of Capsicum annuum ripening fruits is a constitutive process and useful for ripeness index, J Agric Food Chem 48 (2000) 1617-22.

[355] F. Granado, B. Olmedilla, I. Blanco, E. Gil-Martinez, E. Rojas-Hidalgo, Variability in the intercomparison of food carotenoid content data: a user's point of view, Crit Rev Food Sci Nutr 37 (1997) 621-33.

[356] E. Mellado-Ortega, D. Hornero-Mendez, Lutein Esterification in Wheat Flour Increases the Carotenoid Retention and Is Induced by Storage Temperatures, Foods 6 (2017).

[357] A. Pérez-Gálvez, M.I. Minguez-Mosquera, Esterification of xanthophylls and its effect on chemical behavior and bioavailability of carotenoids in the human, Nutrition Research 25 (2005) 631-640.

[358] D.B. Rodriguez-Amaya, A Guide to Carotenoid Analysis in Foods, 2001.

[359] G. Knockaert, L. Lemmens, S. Van Buggenhout, M. Hendrickx, A. Van Loey, Changes in $\beta$ carotene bioaccessibility and concentration during processing of carrot puree, Food Chemistry 133 (2012) 60-67.

[360] A.A. Updike, S.J. Schwartz, Thermal processing of vegetables increases cis isomers of lutein and zeaxanthin, Journal of Agricultural and Food Chemistry 51 (2003) 6184-6190.

[361] A.Z. Mercadante, D.B. Rodriguez-Amaya, Effects of Ripening, Cultivar Differences, and Processing on the Carotenoid Composition of Mango, Journal of Agricultural and Food Chemistry 46 (1998) 128-130.

[362] M.E. O'Neill, Y. Carroll, B. Corridan, B. Olmedilla, F. Granado, I. Blanco, H. van den Berg, I. Hininger, A.-M. Rousell, M. Chopra, S. Southon, D.I. Thurnham, A European carotenoid database to assess carotenoid intakes and its use in a five-country comparative study, $\mathrm{Br} \mathrm{J}$ Nutr 85 (2001) 499-507.

[363] E. Biehler, A.a. Alkerwi, L. Hoffmann, E. Krause, M. Guillaume, M.L. Lair, T. Bohn, Contribution of violaxanthin, neoxanthin, phytoene and phytofluene to total carotenoid intake: Assessment in Luxembourg, Journal of Food Composition and Analysis 25 (2012) 56-65.

[364] D.B. Rodriguez-Amaya, M. Kimura, H.T. Godoy, J. Amaya-Farfan, Updated Brazilian database on food carotenoids: Factors affecting carotenoid composition., J Food Comp Anal 21 (2008) 445-463.

[365] B. Beltran, R. Estevez, C. Cuadrado, S. Jimenez, B. Olmedilla-Alonso, Base de datos de carotenoides para valoracion de la ingesta dietetica de carotenos, xantofilas y de vitamina $A$; Utilizacion en un estudio comparativo del estado nutricional en vitamina $A$ de adultos jovenes, Nutr Hosp 27 (2012) 1334-1343. 
[366] P.M. Bramley, Carotenoid Biosynthesis and Chlorophyll Degradation, in: G.B. Seymour, M. Poole, J.J. Giovannoni, G.A. Tucker (Eds.), The Molecular Biology and Biochemistry of Fruit Ripening, Wiley2013, pp. 76-112.

[367] M. Jenab, S. Salvini, C.H. van Gils, M. Brustad, S. Shakya-Shrestha, B. Buijsse, H. Verhagen, M. Touvier, C. Biessy, P. Wallstrom, K. Bouckaert, E. Lund, M. Waaseth, N. Roswall, A.M. Joensen, J. Linseisen, H. Boeing, E. Vasilopoulou, V. Dilis, S. Sieri, C. Sacerdote, P. Ferrari, J. Manjer, S. Nilsson, A.A. Welch, R. Travis, M.C. Boutron-Ruault, M. Niravong, H.B. Bueno-deMesquita, Y.T. van der Schouw, M.J. Tormo, A. Barricarte, E. Riboli, S. Bingham, N. Slimani, Dietary intakes of retinol, beta-carotene, vitamin $D$ and vitamin $E$ in the European Prospective Investigation into Cancer and Nutrition cohort, Eur J Clin Nutr 63 Suppl 4 (2009) S150-78.

[368] T. Bohn, G.J. McDougall, A. Alegria, M. Alminger, E. Arrigoni, A.M. Aura, C. Brito, A. Cilla, S.N. El, S. Karakaya, M.C. Martinez-Cuesta, C.N. Santos, Mind the gap-deficits in our knowledge of aspects impacting the bioavailability of phytochemicals and their metabolites--a position paper focusing on carotenoids and polyphenols, Mol Nutr Food Res 59 (2015) 130723.

[369] T. Bohn, C. Desmarchelier, L.O. Dragsted, C.S. Nielsen, W. Stahl, R. Ruhl, J. Keijer, P. Borel, Host-related factors explaining interindividual variability of carotenoid bioavailability and tissue concentrations in humans, Mol Nutr Food Res 61 (2017).

[370] K.M. Ranard, S. Jeon, E.S. Mohn, J.C. Griffiths, E.J. Johnson, J.W. Erdman, Jr., Dietary guidance for lutein: consideration for intake recommendations is scientifically supported, Eur J Nutr 56 (2017) 37-42.

[371] Y.M. Huang, H.L. Dou, F.F. Huang, X.R. Xu, Z.Y. Zou, X.M. Lin, Effect of supplemental lutein and zeaxanthin on serum, macular pigmentation, and visual performance in patients with early age-related macular degeneration, Biomed Res Int 2015 (2015) 564738.

[372] P.J. Aggett, Population reference intakes and micronutrient bioavailability: a European perspective, Am J Clin Nutr 91 (2010) 1433S-1437S.

[373] D. Rodriguez-Amaya, Status of carotenoid analytical methods and in vitro assays for the assessment of food quality and health effects, Current Op. Food Sci. 1 (2015) 56-63.

[374] T. Bohn, Bioavailability of Non-Provitamin A Carotenoids, Current Nutrition \& Food Science 4 (2008) 240-258.

[375] J.J. Castenmiller, C.E. West, Bioavailability and bioconversion of carotenoids, Annu Rev Nutr 18 (1998) 19-38.

[376] L. Lemmens, I. Colle, S. Van Buggenhout, P. Palmero, A. Van Loey, M. Hendrickx, Carotenoid bioaccesibility in fruit- and vegetable-based food products as affected by product (micro) structural characteristics and the presente of lipids: A review, Trends Food Sci. Technol. 38 (2014) 125-135.

[377] M. van Lieshout, C.E. West, R.B. van Breemen, Isotopic tracer techniques for studying the bioavailability and bioefficacy of dietary carotenoids, particularly beta-carotene, in humans: a review, Am J Clin Nutr 77 (2003) 12-28.

[378] J.M. Carbonell-Capella, M. Buniowska, F.J. Barba, M.J. Esteve, A. Frígola, Analytical methods for determining bioavailability and bioaccesibility of bioactive compounds from fruits and vegetables: A review, Comprehensive Reviews in Food Science and Food Safety 13 (2014) 155171.

[379] R.K. Saini, S.H. Nile, S.W. Park, Carotenoids from fruits and vegetables: Chemistry, analysis, occurrence, bioavailability and biological activities, Food Res Int 76 (2015) 735-750. 
[380] K. Venema, M. Van Nuenen, M. Smeets-Peeters, M. Minekus, R. Havenaar, TNO's in vitro large intestinal model: an excellent screening tool for functional food and pharmaceutical research, Nutrition Research 24 (2000) 558-564.

[381] M. Minekus, M. Alminger, P. Alvito, S. Ballance, T. Bohn, C. Bourlieu, F. Carriere, R. Boutrou, M. Corredig, D. Dupont, C. Dufour, L. Egger, M. Golding, S. Karakaya, B. Kirkhus, S. Le Feunteun, U. Lesmes, A. Macierzanka, A. Mackie, S. Marze, D.J. McClements, O. Menard, I. Recio, C.N. Santos, R.P. Singh, G.E. Vegarud, M.S. Wickham, W. Weitschies, A. Brodkorb, A standardised static in vitro digestion method suitable for food - an international consensus, Food Funct 5 (2014) 1113-24.

[382] R. Estevez-Santiago, B. Olmedilla-Alonso, I. Fernandez-Jalao, Bioaccessibility of provitamin A carotenoids from fruits: application of a standardised static in vitro digestion method, Food Funct 7 (2016) 1354-66.

[383] F. Granado-Lorencio, I. Blanco-Navarro, B. Perez-Sacristan, E. Hernandez-Alvarez, Biomarkers of carotenoid bioavailability, Food Res Int 99 (2017) 902-916.

[384] P. Trumbo, A.A. Yates, S. Schlicker, M. Poos, Dietary reference intakes: vitamin A, vitamin K, arsenic, boron, chromium, copper, iodine, iron, manganese, molybdenum, nickel, silicon, vanadium, and zinc, J Am Diet Assoc 101 (2001) 294-301.

[385] B.J. Burri, J.S. Chang, T.R. Neidlinger, beta-Cryptoxanthin- and alpha-carotene-rich foods have greater apparent bioavailability than beta-carotene-rich foods in Western diets, Br J Nutr 105 (2011) 212-9.

[386] E.H. Harrison, Mechanisms involved in the intestinal absorption of dietary vitamin A and provitamin A carotenoids, Biochim Biophys Acta 1821 (2012) 70-7.

[387] A. Eroglu, E.H. Harrison, Carotenoid metabolism in mammals, including man: formation, occurrence, and function of apocarotenoids, J Lipid Res 54 (2013) 1719-30.

[388] E. Reboul, Absorption of vitamin A and carotenoids by the enterocyte: focus on transport proteins, Nutrients 5 (2013) 3563-81.

[389] V. Shete, L. Quadro, Mammalian metabolism of beta-carotene: gaps in knowledge, Nutrients 5 (2013) 4849-68.

[390] J. von Lintig, Provitamin A metabolism and functions in mammalian biology, Am J Clin Nutr 96 (2012) 1234S-44S.

[391] K.J. Yeum, R.M. Russell, Carotenoid bioavailability and bioconversion, Annu Rev Nutr 22 (2002) 483-504.

[392] P. Mapelli-Brahm, J. Corte-Real, A.J. Melendez-Martinez, T. Bohn, Bioaccessibility of phytoene and phytofluene is superior to other carotenoids from selected fruit and vegetable juices, Food Chem 229 (2017) 304-311.

[393] C. Chitchumroonchokchai, M.L. Failla, Hydrolysis of zeaxanthin esters by carboxyl ester lipase during digestion facilitates micellarization and uptake of the xanthophyll by Caco-2 human intestinal cells, J Nutr 136 (2006) 588-94.

[394] V. Tyssandier, N. Cardinault, C. Caris-Veyrat, M.J. Amiot, P. Grolier, C. Bouteloup, V. AzaisBraesco, P. Borel, Vegetable-borne lutein, lycopene, and beta-carotene compete for incorporation into chylomicrons, with no adverse effect on the medium-term (3-wk) plasma status of carotenoids in humans, Am J Clin Nutr 75 (2002) 526-34.

[395] G.P. Lobo, J. Amengual, H.N. Li, M. Golczak, M.L. Bonet, K. Palczewski, J. von Lintig, Beta,beta-carotene decreases peroxisome proliferator receptor gamma activity and reduces 
lipid storage capacity of adipocytes in a beta,beta-carotene oxygenase 1-dependent manner, J Biol Chem 285 (2010) 27891-9.

[396] H. Musinovic, M.L. Bonet, N. Granados, J. Amengual, J. von Lintig, J. Ribot, A. Palou, betaCarotene during the suckling period is absorbed intact and induces retinoic acid dependent responses similar to preformed vitamin $A$ in intestine and liver, but not adipose tissue of young rats, Mol Nutr Food Res 58 (2014) 2157-65.

[397] G.P. Lobo, S. Hessel, A. Eichinger, N. Noy, A.R. Moise, A. Wyss, K. Palczewski, J. von Lintig, ISX is a retinoic acid-sensitive gatekeeper that controls intestinal beta,beta-carotene absorption and vitamin A production, FASEB J 24 (2010) 1656-66.

[398] H. van den Berg, R. Faulks, H.F. Granado, J. Hirschberg, B. Olmedilla, G. Sandmann, S. Southon, W. Stahl, The potential for the improvement of carotenoid levels in foods and the likely systemic effects, Journal of the Science of Food and Agriculture 80 (2000) 880-912.

[399] M. Moussa, E. Gouranton, B. Gleize, C.E. Yazidi, I. Niot, P. Besnard, P. Borel, J.F. Landrier, CD36 is involved in lycopene and lutein uptake by adipocytes and adipose tissue cultures, Mol Nutr Food Res 55 (2011) 578-84.

[400] B. Li, P. Vachali, P.S. Bernstein, Human ocular carotenoid-binding proteins, Photochem Photobiol Sci 9 (2010) 1418-25.

[401] R. Kawaguchi, J. Yu, J. Honda, J. Hu, J. Whitelegge, P. Ping, P. Wiita, D. Bok, H. Sun, A membrane receptor for retinol binding protein mediates cellular uptake of vitamin A, Science 315 (2007) 820-5.

[402] D.C. Berry, H. Jacobs, G. Marwarha, A. Gely-Pernot, S.M. O'Byrne, D. DeSantis, M. Klopfenstein, B. Feret, C. Dennefeld, W.S. Blaner, C.M. Croniger, M. Mark, N. Noy, N.B. Ghyselinck, The STRA6 receptor is essential for retinol-binding protein-induced insulin resistance but not for maintaining vitamin $\mathrm{A}$ homeostasis in tissues other than the eye, J Biol Chem 288 (2013) 24528-39.

[403] D.C. Berry, H. Jin, A. Majumdar, N. Noy, Signaling by vitamin A and retinol-binding protein regulates gene expression to inhibit insulin responses, Proc Natl Acad Sci U S A 108 (2011) 4340-5.

[404] P. Alapatt, F. Guo, S.M. Komanetsky, S. Wang, J. Cai, A. Sargsyan, E. Rodriguez Diaz, B.T. Bacon, P. Aryal, T.E. Graham, Liver retinol transporter and receptor for serum retinol-binding protein (RBP4), J Biol Chem 288 (2013) 1250-65.

[405] J. Amengual, M.A. Widjaja-Adhi, S. Rodriguez-Santiago, S. Hessel, M. Golczak, K. Palczewski, J. von Lintig, Two carotenoid oxygenases contribute to mammalian provitamin A metabolism, J Biol Chem 288 (2013) 34081-96.

[406] S. Hessel, A. Eichinger, A. Isken, J. Amengual, S. Hunzelmann, U. Hoeller, V. Elste, W. Hunziker, R. Goralczyk, V. Oberhauser, J. von Lintig, A. Wyss, CMO1 deficiency abolishes vitamin A production from beta-carotene and alters lipid metabolism in mice, J Biol Chem 282 (2007) 33553-61.

[407] J. Amengual, G.P. Lobo, M. Golczak, H.N. Li, T. Klimova, C.L. Hoppel, A. Wyss, K. Palczewski, J. von Lintig, A mitochondrial enzyme degrades carotenoids and protects against oxidative stress, FASEB J 25 (2011) 948-59.

[408] A. Lindqvist, S. Andersson, Biochemical properties of purified recombinant human betacarotene 15,15'-monooxygenase, J Biol Chem 277 (2002) 23942-23948.

[409] G.P. Lobo, A. Isken, S. Hoff, D. Babino, J. von Lintig, BCDO2 acts as a carotenoid scavenger and gatekeeper for the mitochondrial apoptotic pathway, Development 139 (2012) 2966-77. 
[410] J.L. Napoli, Cellular retinoid binding-proteins, CRBP, CRABP, FABP5: Effects on retinoid metabolism, function and related diseases, Pharmacol Ther 173 (2017) 19-33.

[411] N.I. Krinsky, K.J. Yeum, Carotenoid-radical interactions, Biochem Biophys Res Commun 305 (2003) 754-60.

[412] A. Ben-Dor, M. Steiner, L. Gheber, M. Danilenko, N. Dubi, K. Linnewiel, A. Zick, Y. Sharoni, J. Levy, Carotenoids activate the antioxidant response element transcription system, Mol Cancer Ther 4 (2005) 177-86.

[413] J. Fiedor, K. Burda, Potential role of carotenoids as antioxidants in human health and disease, Nutrients 6 (2014) 466-88.

[414] E.J. Johnson, Role of lutein and zeaxanthin in visual and cognitive function throughout the lifespan, Nutr Rev 72 (2014) 605-12.

[415] A. Kaulmann, T. Bohn, Carotenoids, inflammation, and oxidative stress--implications of cellular signaling pathways and relation to chronic disease prevention, Nutr Res 34 (2014) 90729.

[416] V. Sosa, T. Moline, R. Somoza, R. Paciucci, H. Kondoh, L.L. ME, Oxidative stress and cancer: an overview, Ageing Res Rev 12 (2013) 376-90.

[417] F. Bohm, R. Edge, G. Truscott, Interactions of dietary carotenoids with activated (singlet) oxygen and free radicals: potential effects for human health, Mol Nutr Food Res 56 (2012) 205-16.

[418] P. Palozza, S. Serini, F. Di Nicuolo, E. Piccioni, G. Calviello, Prooxidant effects of betacarotene in cultured cells, Mol Aspects Med 24 (2003) 353-62.

[419] W. Siems, I. Wiswedel, C. Salerno, C. Crifo, W. Augustin, L. Schild, C.D. Langhans, O. Sommerburg, Beta-carotene breakdown products may impair mitochondrial functions-potential side effects of high-dose beta-carotene supplementation, J Nutr Biochem 16 (2005) 385-97.

[420] A.J. Young, G.M. Lowe, Antioxidant and prooxidant properties of carotenoids, Arch Biochem Biophys 385 (2001) 20-7.

[421] Y.G. van Helden, J. Keijer, S.G. Heil, C. Pico, A. Palou, P. Oliver, A. Munnia, J.J. Briede, M. Peluso, N.L. Franssen-van Hal, F.J. van Schooten, R.W. Godschalk, Beta-carotene affects oxidative stress-related DNA damage in lung epithelial cells and in ferret lung, Carcinogenesis 30 (2009) 2070-6.

[422] P. Palozza, S. Serini, F. Di Nicuolo, G. Calviello, Modulation of apoptotic signalling by carotenoids in cancer cells, Arch Biochem Biophys 430 (2004) 104-9.

[423] Z.T. Schafer, A.R. Grassian, L. Song, Z. Jiang, Z. Gerhart-Hines, H.Y. Irie, S. Gao, P. Puigserver, J.S. Brugge, Antioxidant and oncogene rescue of metabolic defects caused by loss of matrix attachment, Nature 461 (2009) 109-13.

[424] J. Watson, Oxidants, antioxidants and the current incurability of metastatic cancers, Open Biol 3 (2013) 120144.

[425] M.F. Gregor, G.S. Hotamisligil, Inflammatory mechanisms in obesity, Annu Rev Immunol 29 (2011) 415-45.

[426] L. Marseglia, S. Manti, G. D'Angelo, A. Nicotera, E. Parisi, G. Di Rosa, E. Gitto, T. Arrigo, Oxidative stress in obesity: a critical component in human diseases, Int J Mol Sci 16 (2014) 378-400. 
[427] K. Makki, P. Froguel, I. Wolowczuk, Adipose tissue in obesity-related inflammation and insulin resistance: cells, cytokines, and chemokines, ISRN Inflamm 2013 (2013) 139239.

[428] B.E. Wisse, The inflammatory syndrome: the role of adipose tissue cytokines in metabolic disorders linked to obesity, J Am Soc Nephrol 15 (2004) 2792-800.

[429] E. Karkeni, L. Bonnet, J. Astier, C. Couturier, J. Dalifard, F. Tourniaire, J.F. Landrier, All-transretinoic acid represses chemokine expression in adipocytes and adipose tissue by inhibiting NF-kappaB signaling, J Nutr Biochem 42 (2017) 101-107.

[430] S. Fenni, H. Hammou, J. Astier, L. Bonnet, E. Karkeni, C. Couturier, F. Tourniaire, J.F. Landrier, Lycopene and tomato powder supplementation similarly inhibit high-fat diet induced obesity, inflammatory response, and associated metabolic disorders, Mol Nutr Food Res 61 (2017).

[431] V. Bhuvaneswari, B. Velmurugan, S. Balasenthil, C.R. Ramachandran, S. Nagini, Chemopreventive efficacy of lycopene on 7,12-dimethylbenz[a]anthracene-induced hamster buccal pouch carcinogenesis, Fitoterapia 72 (2001) 865-74.

[432] Y. Sharoni, K. Linnewiel-Hermoni, M. Khanin, H. Salman, A. Veprik, M. Danilenko, J. Levy, Carotenoids and apocarotenoids in cellular signaling related to cancer: a review, Mol Nutr Food Res 56 (2012) 259-69.

[433] X. Zhang, W.E. Zhao, L. Hu, L. Zhao, J. Huang, Carotenoids inhibit proliferation and regulate expression of peroxisome proliferators-activated receptor gamma (PPARgamma) in K562 cancer cells, Arch Biochem Biophys 512 (2011) 96-106.

[434] F. Lian, X.D. Wang, Enzymatic metabolites of lycopene induce Nrf2-mediated expression of phase II detoxifying/antioxidant enzymes in human bronchial epithelial cells, Int J Cancer 123 (2008) 1262-8.

[435] Y. Xia, S. Shen, I.M. Verma, NF-kappaB, an active player in human cancers, Cancer Immunol Res 2 (2014) 823-30.

[436] J. Bastien, C. Rochette-Egly, Nuclear retinoid receptors and the transcription of retinoidtarget genes, Gene 328 (2004) 1-16.

[437] A. Aranda, A. Pascual, Nuclear hormone receptors and gene expression, Physiol Rev 81 (2001) 1269-304.

[438] N. Shaw, M. Elholm, N. Noy, Retinoic acid is a high affinity selective ligand for the peroxisome proliferator-activated receptor beta/delta, J Biol Chem 278 (2003) 41589-92.

[439] M. Rieck, W. Meissner, S. Ries, S. Muller-Brusselbach, R. Muller, Ligand-mediated regulation of peroxisome proliferator-activated receptor (PPAR) beta/delta: a comparative analysis of PPAR-selective agonists and all-trans retinoic acid, Mol Pharmacol 74 (2008) 1269-77.

[440] T.T. Schug, D.C. Berry, N.S. Shaw, S.N. Travis, N. Noy, Opposing effects of retinoic acid on cell growth result from alternate activation of two different nuclear receptors, Cell 129 (2007) 723-33.

[441] N. Bushue, Y.J. Wan, Retinoid pathway and cancer therapeutics, Adv Drug Deliv Rev 62 (2010) 1285-98.

[442] F. Lian, D.E. Smith, H. Ernst, R.M. Russell, X.D. Wang, Apo-10'-lycopenoic acid inhibits lung cancer cell growth in vitro, and suppresses lung tumorigenesis in the $A / J$ mouse model in vivo, Carcinogenesis 28 (2007) 1567-74. 
[443] E. Gouranton, G. Aydemir, E. Reynaud, J. Marcotorchino, C. Malezet, C. Caris-Veyrat, R. Blomhoff, J.F. Landrier, R. Ruhl, Apo-10'-lycopenoic acid impacts adipose tissue biology via the retinoic acid receptors, Biochim Biophys Acta 1811 (2011) 1105-14.

[444] A. Eroglu, D.P. Hruszkewycz, C. dela Sena, S. Narayanasamy, K.M. Riedl, R.E. Kopec, S.J. Schwartz, R.W. Curley, Jr., E.H. Harrison, Naturally occurring eccentric cleavage products of provitamin A beta-carotene function as antagonists of retinoic acid receptors, J Biol Chem 287 (2012) 15886-95.

[445] O. Ziouzenkova, G. Orasanu, G. Sukhova, E. Lau, J.P. Berger, G. Tang, N.I. Krinsky, G.G. Dolnikowski, J. Plutzky, Asymmetric cleavage of beta-carotene yields a transcriptional repressor of retinoid $\mathrm{X}$ receptor and peroxisome proliferator-activated receptor responses, Mol Endocrinol 21 (2007) 77-88.

[446] Y. Shirakura, K. Takayanagi, K. Mukai, H. Tanabe, M. Inoue, beta-cryptoxanthin suppresses the adipogenesis of 3T3-L1 cells via RAR activation, J Nutr Sci Vitaminol (Tokyo) 57 (2011) 42631.

[447] M. Inoue, H. Tanabe, A. Matsumoto, M. Takagi, K. Umegaki, S. Amagaya, J. Takahashi, Astaxanthin functions differently as a selective peroxisome proliferator-activated receptor gamma modulator in adipocytes and macrophages, Biochem Pharmacol 84 (2012) 692-700.

[448] T.R. Breitman, N. Takahashi, Retinoylation of proteins in mammalian cells, Biochem Soc Trans 24 (1996) 723-7.

[449] R. Blomhoff, H.K. Blomhoff, Overview of retinoid metabolism and function, J Neurobiol 66 (2006) 606-30.

[450] Z. Al Tanoury, A. Piskunov, C. Rochette-Egly, Vitamin A and retinoid signaling: genomic and nongenomic effects, J Lipid Res 54 (2013) 1761-75. 


\section{Tables}

Table 1. Bacterial genes and enzymes involved in carotenoid biosynthesis

\begin{tabular}{|c|c|}
\hline Gene & Product (enzyme) \\
\hline crtA & Spheroidene monooxyenase \\
\hline crtB & Phytoene synthase \\
\hline crtC & Hydroxyneurosporene synthase \\
\hline crtD & Methoxyneurosporene desaturase \\
\hline crtE & GGPP synthase \\
\hline crtF & Hydroxyneurosporene-O-methyltransferase \\
\hline crtG & $\beta$-Carotene 2-hydroxylase \\
\hline crtH & Carotene isomerase \\
\hline crtl & Phytoene desaturase (Crtl type) \\
\hline crtL & Lycopene cyclase (CrtL type) \\
\hline$c r t L-b$ & Lycopene $\beta$-cyclase \\
\hline crtL-e, Icy-e & Lycopene $\varepsilon$-cyclase \\
\hline crtM & Dehydrosqualene synthase \\
\hline $\operatorname{ctrN}$ & Dehydrosqualene desaturase, Diapophytoene desaturase \\
\hline crtO & $\beta$-Carotene ketolase \\
\hline crtP & Phytoene desaturase (CtrP type) \\
\hline crtQ & $\zeta$-Carotene desaturase \\
\hline crtR & $\beta$-Carotene hydroxylase \\
\hline crtS & Stress-responsive sigmaB-like protein \\
\hline crtT & Methyltransferase-like protein \\
\hline crtV & Methyltransferase-like protein \\
\hline crtU & $\beta$-Carotene hydrogenase \\
\hline crtW & $\beta$-Carotene ketolase, $\beta$-C-4-oxygenase \\
\hline $\operatorname{crt} X$ & Zeaxanthin glucosylase \\
\hline crtY & Lycopene cyclase (CrtL type) \\
\hline $\operatorname{crtYc}$ & Lycopene cyclase \\
\hline crtYd & Lycopene cyclase \\
\hline crtZ & $\beta$-Carotene hydroxylase \\
\hline
\end{tabular}


Table 2. Common carotenes and xanthophylls in foods and natural sources.

\begin{tabular}{|c|c|c|}
\hline Type & Carotenoid & Natural occurrence \\
\hline \multirow[t]{3}{*}{ Carotenes } & $\begin{array}{l}\alpha \text {-carotene, } \beta \text {-carotene, } \\
\delta \text {-carotene, } \gamma \text {-carotene, } \\
\varepsilon \text {-carotene, } \zeta \text {-carotene }\end{array}$ & $\begin{array}{l}\text { Fruits and vegetables, especially in carrots, sweet } \\
\text { potato, palm tree fruit. } \\
\text { Rose hips are good source for } \gamma \text {-carotene. }\end{array}$ \\
\hline & lycopene, neurosporene & Tomato, water melon and rose hips. \\
\hline & phytofluene, phytoene & Carotenoid-rich fruits, flowers and carrot. \\
\hline \multirow{14}{*}{ Xanthophylls } & antheraxanthin & $\begin{array}{l}\text { Fruits and green vegetables. } \\
\text { Anthers and petals of many yellow flowers. }\end{array}$ \\
\hline & astaxanthin & Fish (salmon) and crustaceans (e.g. lobster). \\
\hline & bixin, norbixin & Annatto (Bixa orellana) seeds. \\
\hline & canthaxanthin & Fungi, cyanobacteria and green algae. \\
\hline & capsanthin, capsorubin & Pepper ripe fruits. \\
\hline & crocetin & Saffron stigmas. \\
\hline & cucurbitaxanthin A & Pumpkin flesh. \\
\hline & lactucaxanthin & Lettuce leaves. \\
\hline & lutein & $\begin{array}{l}\text { Green fruits, vegetables, flowers and cereal } \\
\text { grains (wheat). Also in egg yolk. }\end{array}$ \\
\hline & violaxanthin, neoxanthin & Green fruits, vegetables and flowers. \\
\hline & $\begin{array}{l}\text { luteoxanthin, auroxanthin, } \\
\text { mutatoxanthin }\end{array}$ & $\begin{array}{l}\text { Vegetables and fruits processed under acid } \\
\text { conditions and fermentation. }\end{array}$ \\
\hline & rubixanthin & Rose hips. \\
\hline & zeaxanthin & Corn, potatoes, red pepper. Also in egg yolk. \\
\hline & $\begin{array}{l}\beta \text {-cryptoxanthin, } \\
\alpha \text {-cryptoxanthin }\end{array}$ & $\begin{array}{l}\text { Seeds (corn), flowers and fruits: sweet orange, } \\
\text { tangerine, mango, papaya, persimmon, pepper. }\end{array}$ \\
\hline
\end{tabular}


Table 3. Carotenoid dietary intake from a selection of studies, mean and/or [median]

\begin{tabular}{|c|c|c|c|c|c|c|c|}
\hline \multirow{2}{*}{$\begin{array}{l}\text { Sample (N), } \\
\text { country }\end{array}$} & \multirow{2}{*}{$\begin{array}{c}\text { Women / Men } \\
\text { (age in years) }\end{array}$} & \multicolumn{5}{|c|}{ Dietary intake $(\mathrm{mg} / \mathrm{d}) *$} & \multirow{2}{*}{ Reference } \\
\hline & & $\alpha$-car & $\beta$-car & $\beta$-cryp & Lut/Zea & Lyco & \\
\hline $\mathrm{N}=117$, USA & W (29-39) & $\begin{array}{c}0.75 \\
{[0.55]} \\
\end{array}$ & \begin{tabular}{|c|}
3.34 \\
{$[2.70]$} \\
\end{tabular} & \begin{tabular}{|c|}
0.04 \\
{$[0.03]$} \\
\end{tabular} & \begin{tabular}{|c|}
2.39 \\
{$[1.87]$} \\
\end{tabular} & \begin{tabular}{|c|}
3.35 \\
{$[3.01]$} \\
\end{tabular} & [1] \\
\hline $\mathrm{N}=547$, USA & $\begin{array}{l}346 \text { W (67-93) } \\
201 \text { M (68-91) }\end{array}$ & $\begin{array}{c}0.86 \\
{[0.56]} \\
0.66 \\
{[0.44]}\end{array}$ & $\begin{array}{c}4.21 \\
{[3.74]} \\
3.52 \\
{[2.90]}\end{array}$ & \begin{tabular}{|c}
0.08 \\
{$[0.05]$} \\
0.06 \\
{$[0.05]$}
\end{tabular} & \begin{tabular}{|c|}
3.09 \\
{$[2.52]$} \\
2.68 \\
{$[2.30]$}
\end{tabular} & $\begin{array}{c}7.00 \\
{[5.65]} \\
7.64 \\
{[6.40]}\end{array}$ & [2] \\
\hline $\mathrm{N}=64$, Ireland & $\begin{array}{l}\text { W (25-45) } \\
W(>65) \\
M(25-45) \\
M(>65)\end{array}$ & $\begin{array}{l}2.43 \\
1.22 \\
2.29 \\
1.21\end{array}$ & $\begin{array}{l}8.80 \\
5.50 \\
8.08 \\
5.28 \\
\end{array}$ & $\begin{array}{l}0.73 \\
0.32 \\
0.47 \\
0.30\end{array}$ & $\begin{array}{l}2.62 \\
2.12 \\
2.32 \\
1.88 \\
\end{array}$ & $\begin{array}{l}8.05 \\
4.62 \\
7.64 \\
2.03\end{array}$ & [3] \\
\hline $\mathrm{N}=280$, USA & W, M (18-50) & & 2.94 & & 1.10 & 8.37 & [4] \\
\hline $\begin{array}{l}\mathrm{N}=75, \text { France } \\
\mathrm{N}=65, \mathrm{~N} \text {. Ireland } \\
\mathrm{N}=73 \text {, Ireland } \\
\mathrm{N}=72 \text {, Holland } \\
\mathrm{N}=64 \text {, Spain }\end{array}$ & $\begin{array}{l}\text { W, M (25-45) } \\
\text { W, M (25-45) } \\
\text { W, M (25-45) } \\
\text { W, M (25-45) } \\
\text { W, M (25-45) }\end{array}$ & $\begin{array}{l}0.74 \\
1.04 \\
1.23 \\
0.68 \\
0.29 \\
\end{array}$ & $\begin{array}{l}5.84 \\
5.55 \\
5.16 \\
4.35 \\
2.96 \\
\end{array}$ & $\begin{array}{l}0.45 \\
0.99 \\
0.78 \\
0.97 \\
1.36\end{array}$ & $\begin{array}{l}2.50 \\
1.59 \\
1.56 \\
2.01 \\
3.25\end{array}$ & $\begin{array}{l}4.75 \\
5.01 \\
4.43 \\
4.86 \\
1.64 \\
\end{array}$ & [5] \\
\hline $\begin{array}{l}\mathrm{N}=459, \text { Costa } \\
\text { Rica }\end{array}$ & $\begin{array}{l}115 \mathrm{~W}(59 \pm 10) \\
344 \mathrm{M}(56 \pm 11)\end{array}$ & $\begin{array}{l}0.73 \\
0.45\end{array}$ & $\begin{array}{l}4.67 \\
3.41\end{array}$ & $\begin{array}{l}0.55 \\
0.38\end{array}$ & $\begin{array}{l}2.89 \\
2.41\end{array}$ & $\begin{array}{l}5.77 \\
5.45\end{array}$ & [6] \\
\hline $\mathrm{N}=59$, USA & W, M (65-87) & 0.64 & 4.60 & 0.07 & 3.81 & 9.43 & [7] \\
\hline $\mathrm{N}=98$, USA & W, M (45-73) & & 4.48 & & 1.79 & 8.29 & [8] \\
\hline $\mathrm{N}=12741$, France & $\begin{array}{l}W(35-60) \\
M(35-60)\end{array}$ & & $\begin{array}{l}3.96 \\
3.92 \\
\end{array}$ & & & & [9] \\
\hline $\begin{array}{l}\mathrm{N}=402, \mathrm{USA} \\
\text { (African-American) }\end{array}$ & $\begin{array}{l}247 \mathrm{~W}(34-84) \\
155 \mathrm{M}(34-84)\end{array}$ & $\begin{array}{l}{[0.25]} \\
{[0.33]}\end{array}$ & \begin{tabular}{|l|}
{$[2.21]$} \\
{$[2.21]$} \\
\end{tabular} & $\begin{array}{l}{[0.13]} \\
{[0.11]}\end{array}$ & $\begin{array}{l}1.93] \\
{[1.85]} \\
\end{array}$ & \begin{tabular}{|l|}
{$[2.60]$} \\
{$[3.16]$} \\
\end{tabular} & [10] \\
\hline $\mathrm{N}=262$, USA & $\mathrm{W}, \mathrm{M}$ & 0.19 & 1.73 & 0.12 & 3.96 & 4.75 & [11] \\
\hline $\mathrm{N}=470, \mathrm{USA}^{\mathrm{a}}$ & $\begin{array}{l}\text { W (40-69) } \\
M(40-69)\end{array}$ & $\begin{array}{l}0.57 \\
0.50\end{array}$ & $\begin{array}{l}3.51 \\
2.91\end{array}$ & $\begin{array}{l}0.14 \\
0.13\end{array}$ & $\begin{array}{l}2.40 \\
2.17\end{array}$ & $\begin{array}{l}4.22 \\
5.64\end{array}$ & [12] \\
\hline $\mathrm{N}=159$, Sweden & W (56-75) & $\begin{array}{c}1.03 \\
{[0.65]}\end{array}$ & $\begin{array}{c}3.47 \\
{[3.07]}\end{array}$ & $\begin{array}{c}0.46 \\
{[0.33]}\end{array}$ & $\begin{array}{l}2.64 \\
{[2.5]}\end{array}$ & $\begin{array}{c}2.15 \\
{[1.91]}\end{array}$ & [13] \\
\hline $\mathrm{N}=108$, Spain & $\begin{array}{l}54 \mathrm{~W} \\
54 \mathrm{M} \\
54(20-35) \\
54(45-65) \\
\end{array}$ & & & & $\begin{array}{l}1.28 \\
1.05 \\
1.00 \\
1.34 \\
\end{array}$ & & [14] \\
\hline $\begin{array}{l}\mathrm{N}=50, \text { Dominican } \\
\text { Republic }\end{array}$ & W, M (22-69) & $\begin{array}{c}0.7 \\
{[0.61]}\end{array}$ & $\begin{array}{c}2.7 \\
{[1.62]} \\
\end{array}$ & $\begin{array}{c}0.22 \\
{[0.08]}\end{array}$ & \begin{tabular}{|c|}
1.33 \\
{$[0.72]$} \\
\end{tabular} & \begin{tabular}{|c|}
1.46 \\
{$[1.09]$} \\
\end{tabular} & [15] \\
\hline $\mathrm{N}=91$, Australia & W (18-70) & {$[2.0]$} & {$[6.87]$} & & {$[2.28]$} & {$[5.05]$} & [16] \\
\hline $\begin{array}{l}\mathrm{N}=96335, \text { USA \& } \\
\text { Canada a }(25 \% \\
\text { African-American) }\end{array}$ & W, M (50-90) & 1.07 & 6.67 & 0.21 & 4.11 & 4.48 & [17] \\
\hline & Fron & Natior & nal Surv & & & & \\
\hline $\begin{array}{l}\mathrm{N}=3000, \text { Spain } \\
\text { Years: } 2009-2010\end{array}$ & $\begin{array}{l}\text { W, M (18-64) } \\
\text { From total diet } \\
\text { From F+V intake }\end{array}$ & $\begin{array}{l}0.27 \\
0.27\end{array}$ & $\begin{array}{l}1.46 \\
1.31\end{array}$ & $\begin{array}{l}0.32 \\
0.22\end{array}$ & $\begin{array}{l}1.24 \\
0.84 \\
\end{array}$ & $\begin{array}{c}3.06 \\
3.0 \\
\end{array}$ & {$[18,19]$} \\
\hline $\begin{array}{l}\mathrm{N}=55950, \text { Brazil } \\
\text { Years: } 2008-2010\end{array}$ & $\begin{array}{l}\mathrm{W}, \mathrm{M}(\geq 10) \\
\quad \text { From F+V intake }\end{array}$ & $0.16 *$ & $0.92 *$ & $0.16^{*}$ & $0.83 *$ & $0.83 *$ & [20] \\
\hline $\begin{array}{l}\text { USA } \\
\text { Years: 2009-2010 }\end{array}$ & $\begin{array}{l}\mathrm{W}, \mathrm{M}(\geq 20) \\
\quad \text { From total diet }\end{array}$ & 0.4 & 1.9 & 0.2 & 1.4 & 1.4 & [21] \\
\hline
\end{tabular}


* Dietary assessment method: food frequency questionnaire, except in [12]: diet history questionnaire; $[11,15,18,19,21]$ : $24 \mathrm{~h}$ dietary recall; [18, 19]: 3-day diet diary; [20]: 2-day records.

${ }^{a}(\mathrm{mg} / \mathrm{d})$ : geometric mean

[1] L.C. Yong, M.R. Forman, G.R. Beecher, B.I. Graubard, W.S. Campbell, M.E. Reichman, P.R. Taylor, E. Lanza, J.M. Holden, J.T. Judd, Relationship between dietary intake and plasma concentrations of carotenoids in premenopausal women: application of the USDA-NCl carotenoid food-composition database, Am J Clin Nutr 60 (1994) 223-30.

[2] K.L. Tucker, H. Chen, S. Vogel, P.W. Wilson, E.J. Schaefer, C.J. Lammi-Keefe, Carotenoid intakes, assessed by dietary questionnaire, are associated with plasma carotenoid concentrations in an elderly population, J Nutr 129 (1999) 438-45.

[3] Y.L. Carroll, B.M. Corridan, P.A. Morrissey, Carotenoids in young and elderly healthy humans: dietary intakes, biochemical status and diet-plasma relationships, Eur J Clin Nutr 53 (1999) 644-53.

[4] J. Curran-Celentano, B.R. Hammond, Jr., T.A. Ciulla, D.A. Cooper, L.M. Pratt, R.B. Danis, Relation between dietary intake, serum concentrations, and retinal concentrations of lutein and zeaxanthin in adults in a Midwest population, Am J Clin Nutr 74 (2001) 796-802.

[5] B. Olmedilla, F. Granado, S. Southon, A.J. Wright, I. Blanco, E. Gil-Martinez, H. Berg, B. Corridan, A.M. Roussel, M. Chopra, D.I. Thurnham, Serum concentrations of carotenoids and vitamins $A, E$, and $C$ in control subjects from five European countries, Br J Nutr 85 (2001) 227-38.

[6] A. El-Sohemy, A. Baylin, E. Kabagambe, A. Ascherio, D. Spiegelman, H. Campos, Individual carotenoid concentrations in adipose tissue and plasma as biomarkers of dietary intake, Am J Clin Nutr 76 (2002) $172-9$.

[7] C.C. Tangney, J.L. Bienias, D.A. Evans, M.C. Morris, Reasonable estimates of serum vitamin E, vitamin C, and beta-cryptoxanthin are obtained with a food frequency questionnaire in older black and white adults, J Nutr 134 (2004) 927-34.

[8] J.D. Burke, J. Curran-Celentano, A.J. Wenzel, Diet and serum carotenoid concentrations affect macular pigment optical density in adults 45 years and older, J Nutr 135 (2005) 1208-14.

[9] H. Faure, P. Preziosi, A.M. Roussel, S. Bertrais, P. Galan, S. Hercberg, A. Favier, Factors influencing blood concentration of retinol, alpha-tocopherol, vitamin $\mathrm{C}$, and beta-carotene in the French participants of the SU.VI.MAX trial, Eur I Clin Nutr 60 (2006) 706-17.

[10] S.A. Talegawkar, E.J. Johnson, T.C. Carithers, H.A. Taylor, M.L. Bogle, K.L. Tucker, Carotenoid intakes, assessed by food-frequency questionnaires (FFQs), are associated with serum carotenoid concentrations in the Jackson Heart Study: validation of the Jackson Heart Study Delta NIRI Adult FFQs, Public Health Nutr 11 (2008) 989-97.

[11] L. Arab, M.C. Cambou, N. Craft, K. Wesseling-Perry, P. Jardack, A. Ang, Racial differences in correlations between reported dietary intakes of carotenoids and their concentration biomarkers, Am J Clin Nutr 93 (2011) 1102-8.

[12] S.M. George, F.E. Thompson, D. Midthune, A.F. Subar, D. Berrigan, A. Schatzkin, N. Potischman, Strength of the relationships between three self-reported dietary intake instruments and serum carotenoids: the Observing Energy and Protein Nutrition (OPEN) Study, Public Health Nutr 15 (2012) $1000-7$.

[13] A. Wawrzyniak, J. Hamulka, E. Friberg, A. Wolk, Dietary, anthropometric, and lifestyle correlates of serum carotenoids in postmenopausal women, Eur J Nutr 52 (2013) 1919-26.

[14] B. Olmedilla-Alonso, B. Beltran-de-Miguel, R. Estevez-Santiago, C. Cuadrado-Vives, Markers of lutein and zeaxanthin status in two age groups of men and women: dietary intake, serum concentrations, lipid profile and macular pigment optical density, Nutr J 13 (2014) 52.

[15] M. Duran-Cabral, I. Fernandez-Jalao, R. Estevez-Santiago, B. Olmedilla Alonso, Assessment of individual carotenoid and vitamin A dietary intake in overweight and obese Dominican subjects, Nutr Hosp 34 (2017) 407-15.

[16] K. Pezdirc, M.J. Hutchesson, R. Whitehead, G. Ozakinci, D. Perrett, C.E. Collins, Fruit, Vegetable and Dietary Carotenoid Intakes Explain Variation in Skin-Color in Young Caucasian Women: A CrossSectional Study, Nutrients 7 (2015) 5800-15.

[17] G.E. Fraser, K. Jaceldo-Siegl, S.M. Henning, J. Fan, S.F. Knutsen, E.H. Haddad, J. Sabate, W.L. Beeson, H. Bennett, Biomarkers of Dietary Intake Are Correlated with Corresponding Measures from Repeated 
Dietary Recalls and Food-Frequency Questionnaires in the Adventist Health Study-2, J Nutr 146 (2016) 586-94.

[18] R. Estevez-Santiago, B. Beltran-de-Miguel, B. Olmedilla-Alonso, Assessment of dietary lutein, zeaxanthin and lycopene intakes and sources in the Spanish survey of dietary intake (2009-2010), Int J Food Sci Nutr 67 (2016) 305-13.

[19] B. Beltran-de-Miguel, R. Estevez-Santiago, B. Olmedilla-Alonso, Assessment of dietary vitamin A intake (retinol, alpha-carotene, beta-carotene, beta-cryptoxanthin) and its sources in the National Survey of Dietary Intake in Spain (2009-2010), Int J Food Sci Nutr 66 (2015) 706-12.

[20] L. Vargas-Murga, V.V. de Rosso, A.Z. Mercadante, O.-A. B., Fruits and vegetables in the Brazilian Household Budget Survey (2008-2009): carotenoid content and assessment of individual carotenoid intake., J Food Comp Anal 50 (2016) 88-96.

[21] NHANES, What We Eat in (2009-2010), https://www.ars.usda.gov/ARSUserFiles/80400530/pdf/0910/tables_1-40_2009-2010.pdf. 


\section{Figures}

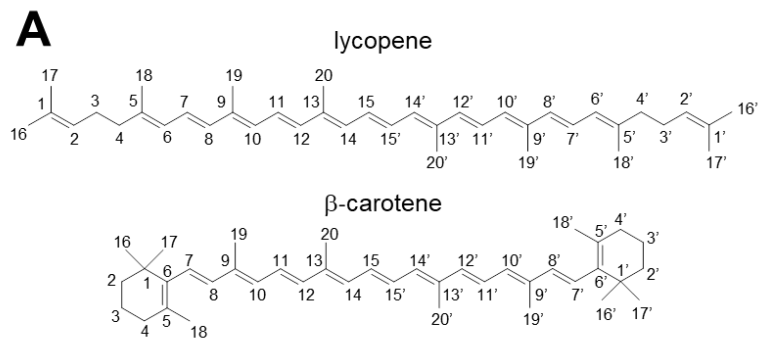

B

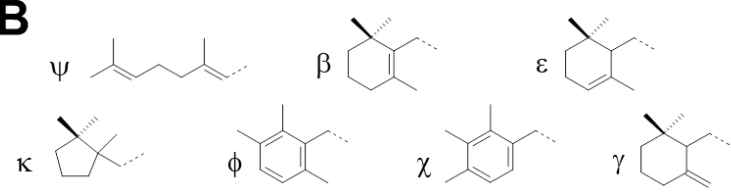

Figure 1. Structural features of carotenoids. A, Numbering of carbon atoms in an acyclic (lycopene) and a cyclic ( $\beta$-carotene) carotenoid. B, End groups present in carotenoid molecules.

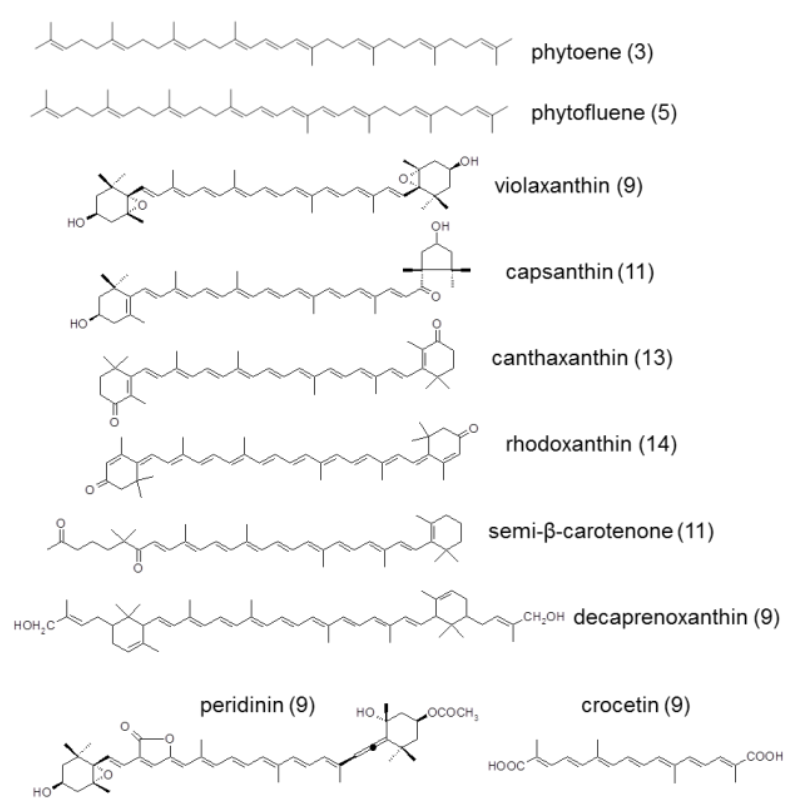

Figure 2. Chemical structures of some carotenoids. Numbers in parentheses indicate the number of conjugated double bonds. Only phytoene and phytofluene are carotenes. 
A
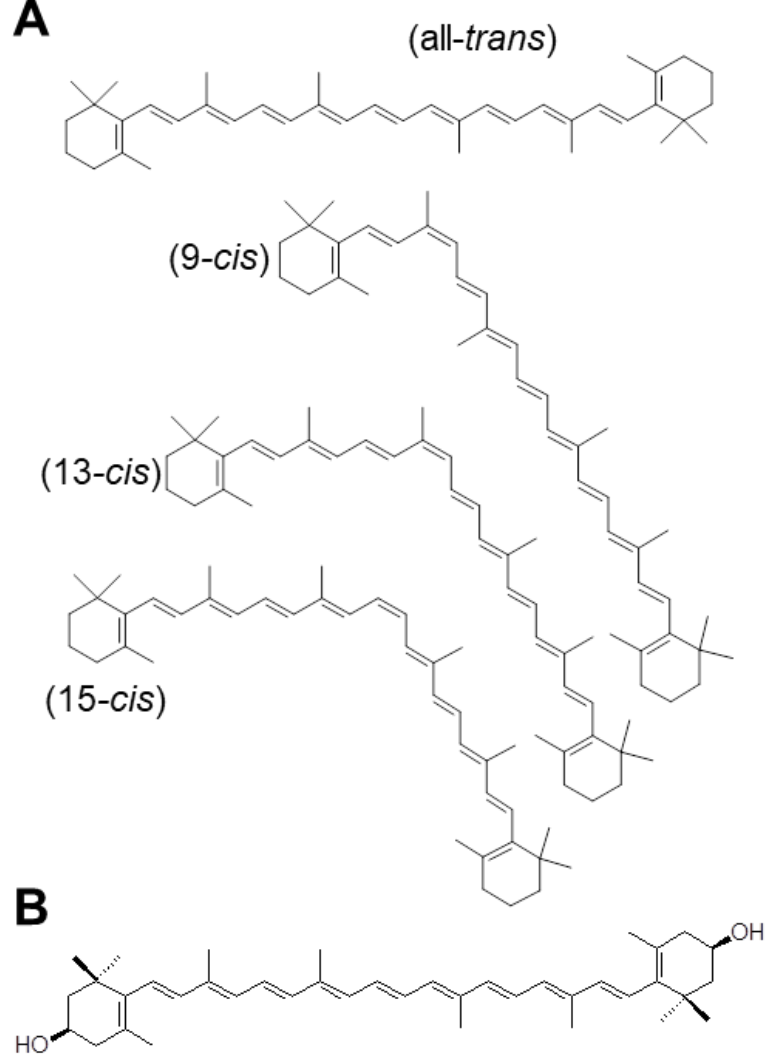

$\left(3 R, 3^{\prime} R\right)$

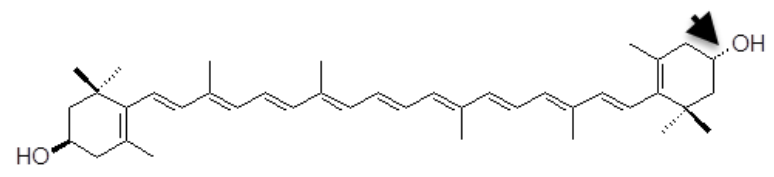

$(3 R, 3$ 'S) meso-zeaxanthin

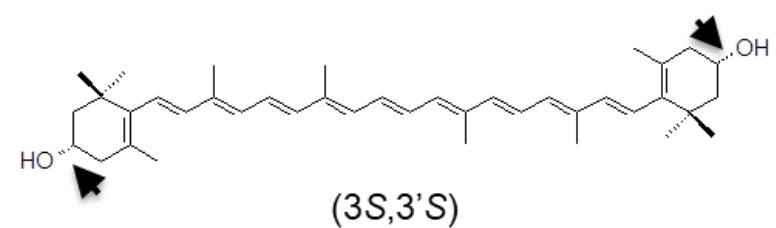

Figure 3. Chemical structure carotenoid isomers. A, Geometrical isomers of $\beta$-carotene. B, Optical isomers of zeaxanthin. Arrows mark $S$ bonds. 


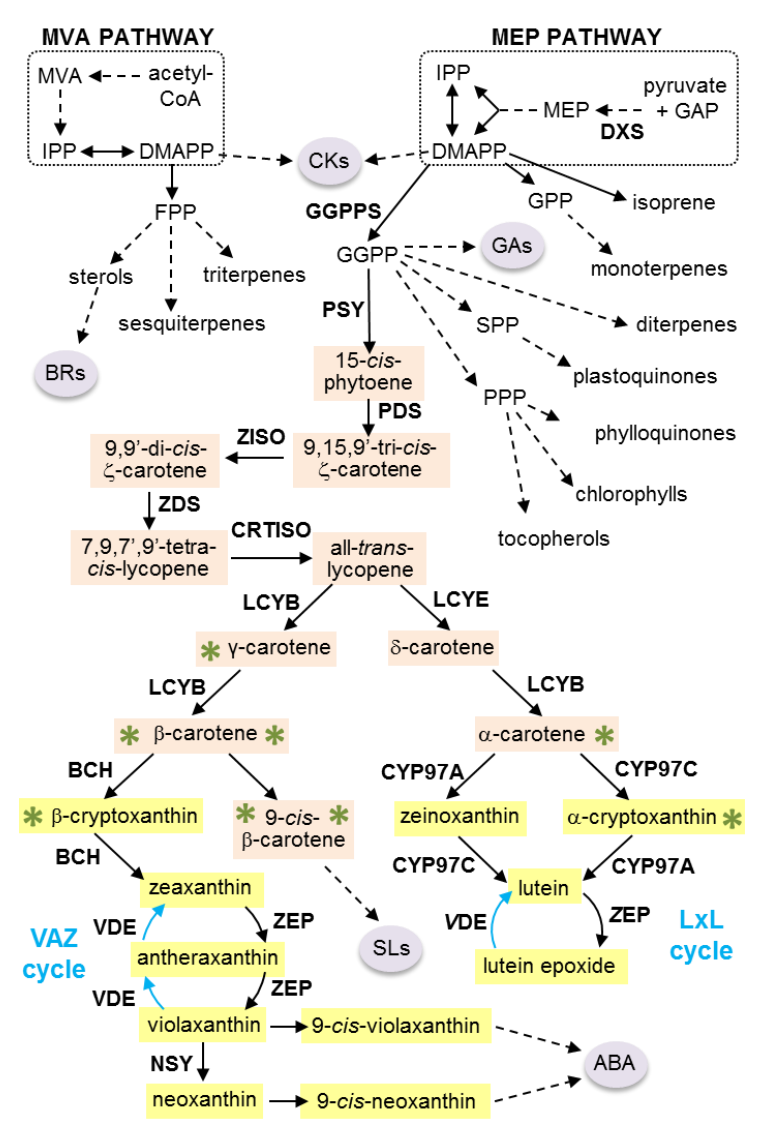

Figure 4. Carotenoid biosynthesis and related isoprenoid pathways in plants. Carotenes are boxed in orange and xanthophylls in yellow. Xanthophyll cycles identified in plants are also shown (blue arrows represent the reactions induced by high light). The presence of unsubstituted $\beta$ rings in provitamin A carotenoids is marked with green asterisks. Hormones are marked with circles (ABA, abscisic acid; BRs, brassinosteroids; CKs, cytokinins; GAs, gibberellins; SLs, strigolactones). Dashed lines represent multiple steps. GAP, glyceraldehyde 3-phosphate; MEP, methylerythritol 4-phosphate; MVA, mevalonic acid; IPP, isopentenyl diphosphate; DMAPP, dimethylallyl diphosphate; GPP, geranyl diphosphate; FPP, farnesyl diphosphate; GGPP, geranylgeranyl diphosphate; SPP, slanesyl diphosphate; PPP, phytyl diphosphate. Enzymes are shown in bold: DXS, deoxyxylulose 5-phosphate synthase; GGPPS, GGPP synthase; PSY, phytoene synthase; PDS,

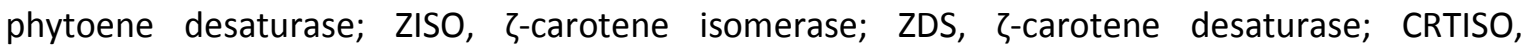
carotenoid isomerase; LCYE, lycopene $\varepsilon$-cyclase; LCYB, lycopene $\beta$-cyclase; CYP97C, carotene $\varepsilon$ hydroxylase; CYP97A, cytochrome $\mathrm{P} 450$ carotene $\beta$-hydroxylase; $\mathrm{BCH}$, non-heme di-iron carotene $\beta$-hydroxylase; ZEP, zeaxanthin epoxidase; VDE, violaxanthin de-epoxidase; NSY, neoxanthin synthase. 


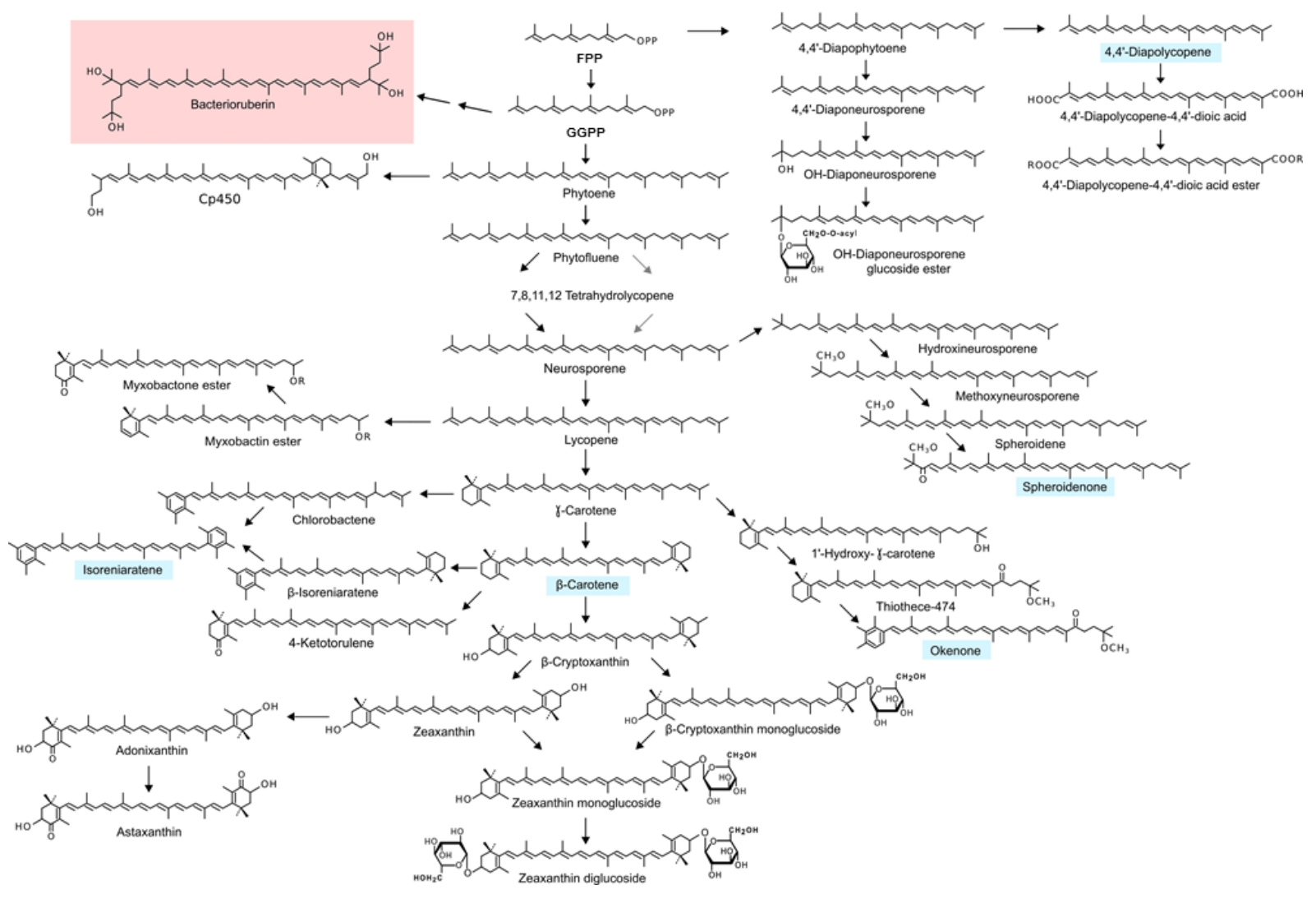

Figure 5. Carotenoid biosynthesis pathways in bacteria and archaea. C50 bacterioruberin, synthesized mainly by archaea, is highlighted in pink. Representative intermediates and products of the main pathways cited in the text are shown (highlighted in light blue). Glucoside esters of zeaxanthin, b-cryptoxanthin and $\mathrm{OH}$-diaponeurosporene are also included.

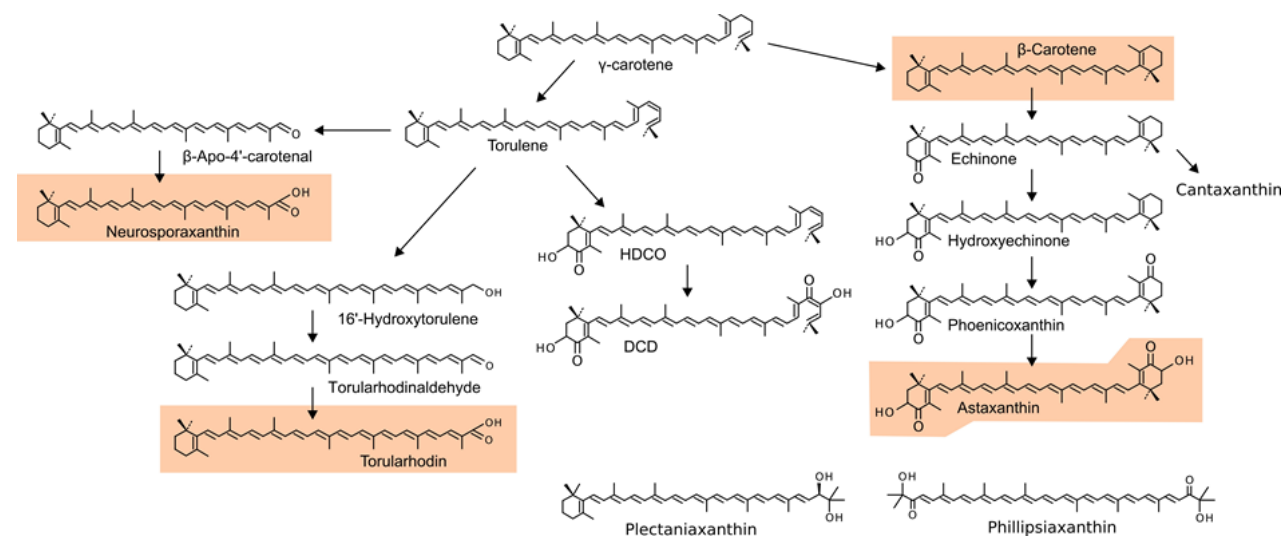

Figure 6. Carotenoid biosynthesis pathways in fungi. Major steps from $\gamma$-carotene to produce astaxanthin, neurosporaxanthin, torularhodin, and 3,3'-dihydroxy- $\beta, \psi$-carotene- $4,4^{\prime}$ dione (DCD) are shown. The most abundant carotenoids in fungi are boxed in orange. DCD and 3-hydroxy$3^{\prime} 4^{\prime}$-didehydro- $\beta, \psi$-caroten-4-one. (HDCO) are side products of astaxanthin biosynthesis in $X$. dendrorhous. Plectaniaxanthin and phillipsiaxanthin are unusual carotenoids identified in Dioszegia sp. and Phillipsia carminea. 


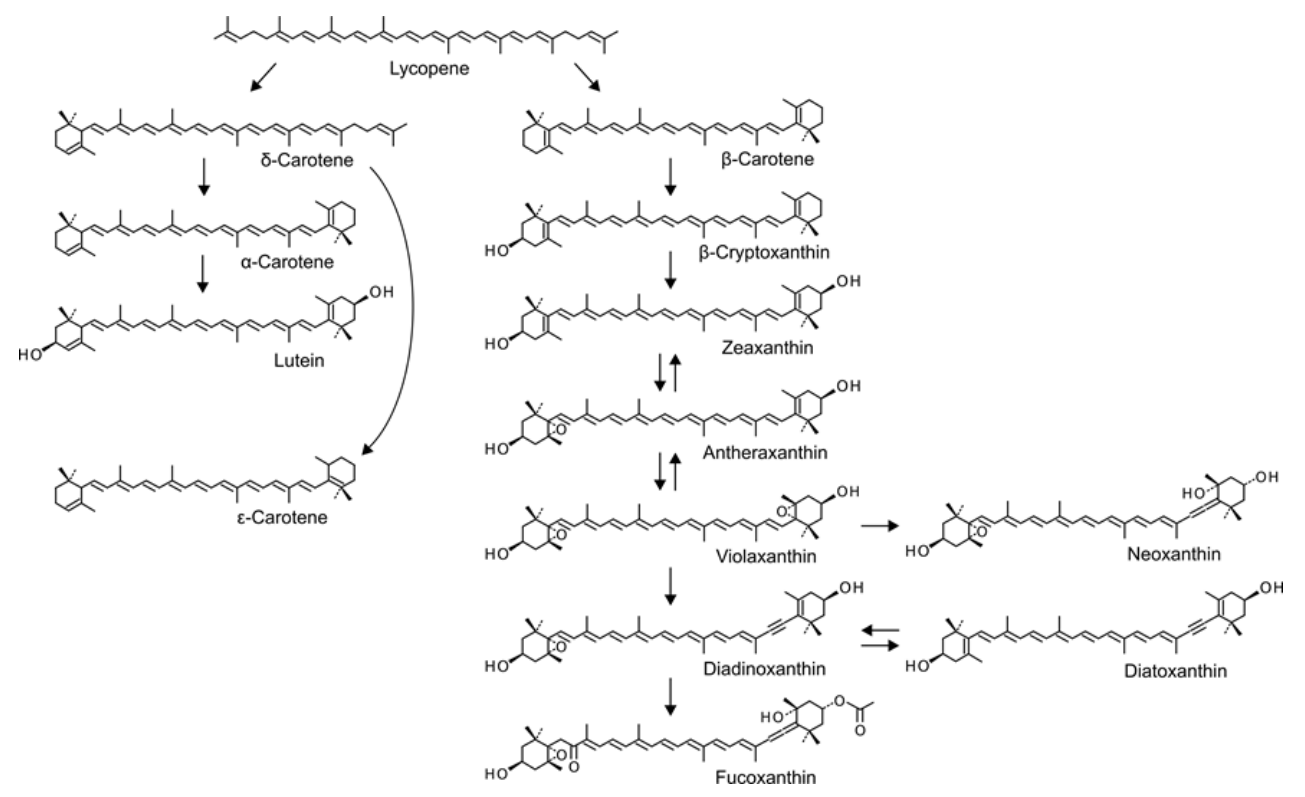

Figure 7. Carotenoid biosynthesis pathways in microalgae. Only biosynthetic steps from lycopene are shown. 


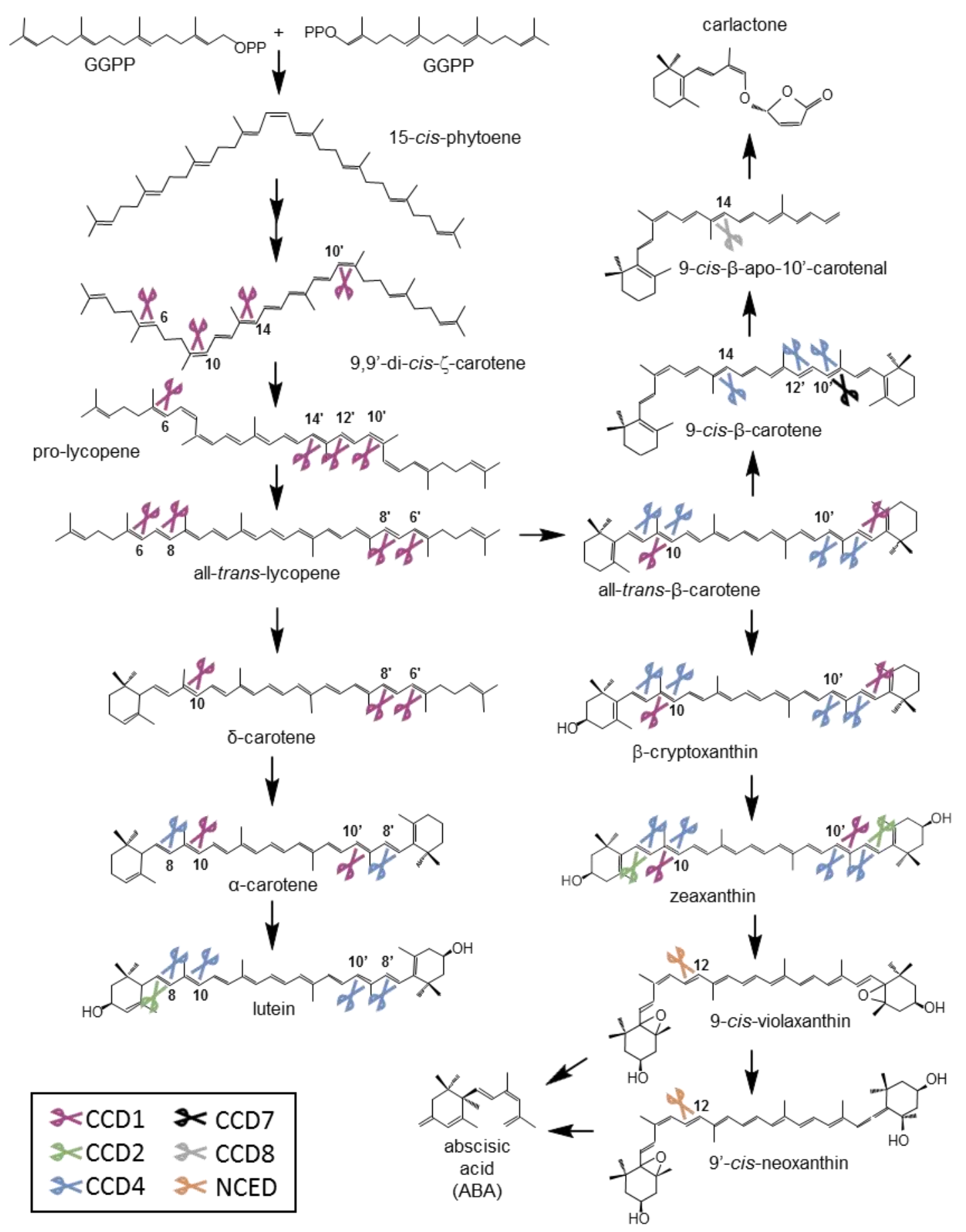

Figure 8. Cleavage of carotenoid substrates by plant CCD enzymes. Scissors indicate the positions cleaved by members of the enzyme families shown in the box. 


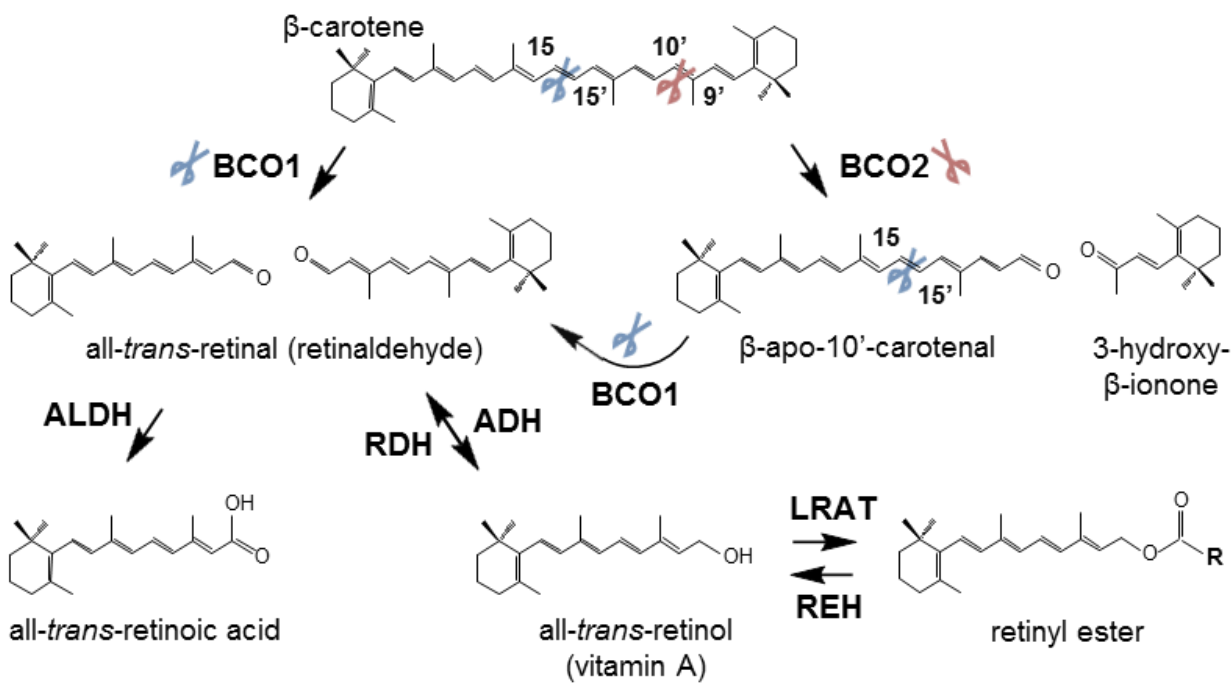

Figure 9. Retinoid biosynthesis pathways in animal cells. The enzyme $\beta$-carotene 15,15'oxygenase 1 (BCO1) cleaves 15,15' double bonds (shown in blue), whereas BCO2 cleaves $9^{\prime}, 10^{\prime}$ double bonds (shown in red). ADH, alcohol dehydrogenase; ALDH, aldehyde dehydrogenase; LRAT, lecithin:retinol acyltransferase; RDH, retinol dehydrogenase; REH, retinyl ester hydrolase. 


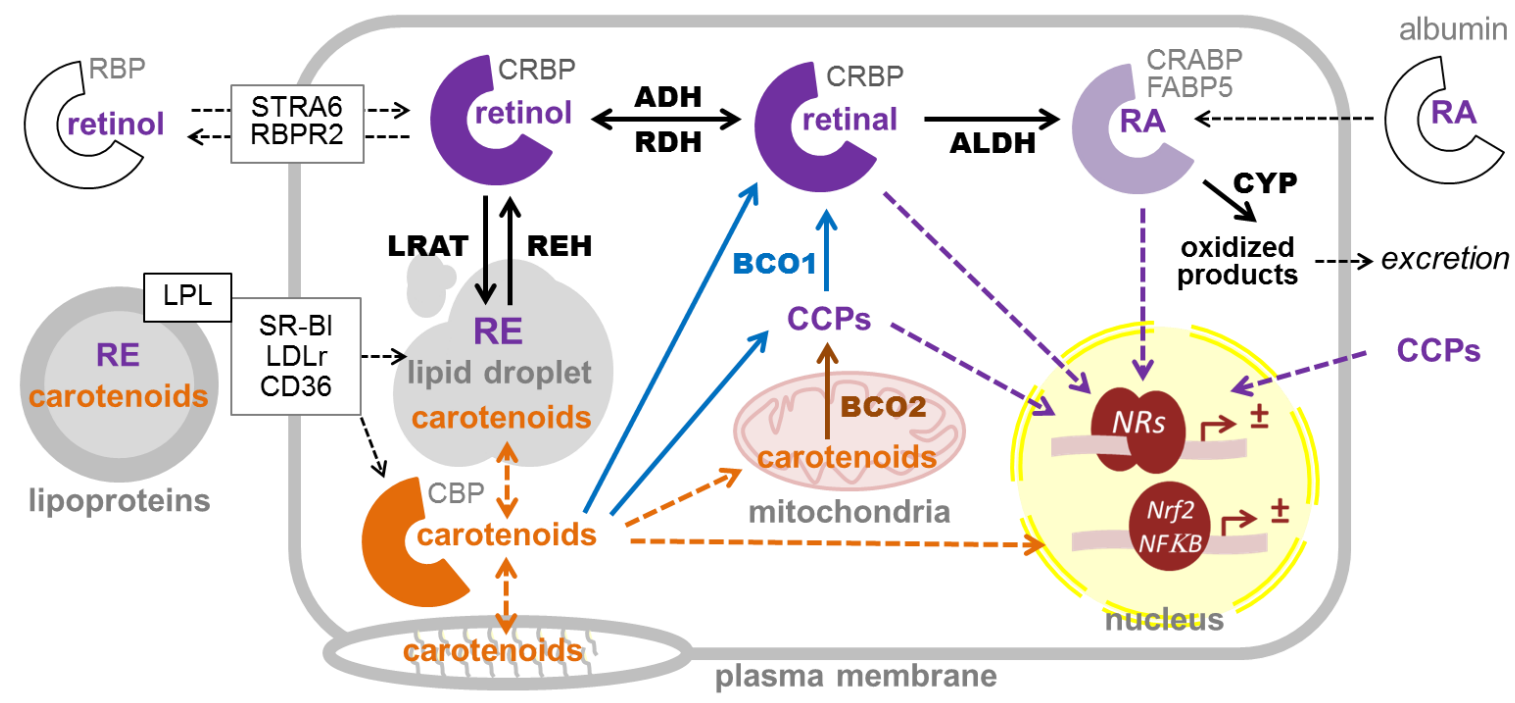

Figure 10. Overview of cellular carotenoid metabolism and interaction with retinoid metabolism in mammal cells. Carotenoids and retinyl esters (RE) contained in circulating lipoproteins are internalized in cells through the action of different lipoprotein receptors (SR-BI, LDLr), membrane transporters (CD36), and lipoprotein lipase (LPL). Within the cells, carotenoids associate to carotenoid-binding proteins (CBP) and incorporate into cytoplasmic lipid droplets and plasma and mitochondria membranes. Symmetric cleavage of provitamin A carotenoids such as $\beta$-carotene by $\mathrm{BCO} 1$ directly renders retinal. Carotenoids are also asymmetrically cleaved through the action of mitochondrial BCO2. Carotenoid cleavage products (CCPs) can be converted into retinal by $\mathrm{BCO} 1$. Retinal is also produced from retinol. Circulating retinol bound to retinol binding protein (RBP) is internalized in cells through the action of specific surface receptors (STRA6, RBPR2) or by diffusion across the plasma membrane. Inside the cell, retinol binds to the cellular retinol binding protein (CRBP) and can be stored as RE in lipid droplets upon esterification to fatty acids by lecithin:retinol acyltransferase (LRAT). RE hydrolase (REH) releases retinol from RE. Retinol and retinal can be interconverted by reversible oxidization catalyzed by short-chain dehydrogenase/reductases with retinol dehydrogenase (RDH) activity or medium-chain alcohol dehydrogenases (ADH). Retinal is further oxidized irreversibly to retinoic acid (RA) by the action of aldehyde dehydrogenases (ALDH). RA is transferred to the nucleus in association with specific intracellular lipid binding proteins (CRABP and FABP5) or can be catabolized by cytochrome P450 enzymes (CYP) to more oxidized products that are eventually eliminated from the cell. Circulating albumin-bound RA (and likely other CCPs) can be taken up by cells. Intact carotenoids, retinoids, and other CCPs can modify gene expression through physical and/or functional interaction with nuclear receptors (NRs) and other transcription factors. They can play additional functions as light filters, antioxidants and prooxidants, and have other effects not directly related to the regulation of gene expression. 